\title{
The Daniel K. Inouye Solar Telescope - Observatory Overview
}

Thomas R. Rimmele ${ }^{1}$ (D) $\cdot$ Mark Warner ${ }^{1}$ - Stephen L. Keil ${ }^{1}$. Philip R. Goode ${ }^{2}$. Michael Knölker ${ }^{3}$ - Jeffrey R. Kuhn ${ }^{4}$ - Robert R. Rosner ${ }^{5}$ - Joseph P. McMullin ${ }^{1}$. Roberto Casini $^{3}$ - Haosheng Lin ${ }^{4}$ Friedrich Wöger ${ }^{1}$. Oskar von der Lühe ${ }^{6}$. Alexandra Tritschler ${ }^{1}$ - Alisdair Davey ${ }^{1}$ - Alfred de Wijn ${ }^{3}$ - David F. Elmore ${ }^{1}$.

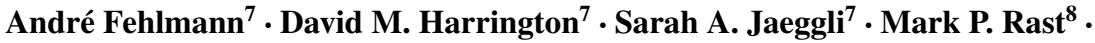
Thomas A. Schad ${ }^{7}$ - Wolfgang Schmidt ${ }^{6}$ Mihalis Mathioudakis ${ }^{9}$. Donald L. Mickey ${ }^{4}$. Tetsu Anan ${ }^{7}$. Christian Beck ${ }^{7}$ - Heather K. Marshall ${ }^{7}$ - Paul F. Jeffers ${ }^{7}$. Jacobus M. Oschmann Jr. ${ }^{1}$ Andrew Beard ${ }^{1}$ - David C. Berst ${ }^{1} \cdot$ Bruce A. Cowan $^{1}$. Simon C. Craig ${ }^{7}$. Eric Cross ${ }^{1}$ - Bryan K. Cummings ${ }^{7}$. Colleen Donnelly ${ }^{7}$. Jean-Benoit de Vanssay ${ }^{7}$. Arthur D. Eigenbrot ${ }^{1}$ • Andrew Ferayorni ${ }^{1}$. Christopher Foster $^{7}$. Chriselle Ann Galapon ${ }^{7}$. Christopher Gedrites ${ }^{7}$. Kerry Gonzales $^{1}$ - Bret D. Goodrich ${ }^{1}$ Brian S. Gregory ${ }^{1}$ - Stephanie S. Guzman ${ }^{7}$. Stephen Guzzo $^{7}$ - Steve Hegwer ${ }^{1}$. Robert P. Hubbard ${ }^{1}$. John R. Hubbard ${ }^{1}$. Erik M. Johansson ${ }^{1}$ • Luke C. Johnson ${ }^{1}$. Chen Liang ${ }^{1}$ - Mary Liang ${ }^{7}$. Isaac McQuillen ${ }^{1}$. Christopher Mayer ${ }^{7} \cdot$ Karl Newman $^{7}$ - Brialyn Onodera ${ }^{7}$. LeEllen Phelps ${ }^{1}$ - Myles M. Puentes ${ }^{7}$. Christopher Richards ${ }^{1} \cdot$ Lukas M. Rimmele $^{1}$. Predrag Sekulic $^{7}$ - Stephan R. Shimko ${ }^{7}$ - Brett E. Simison ${ }^{7}$ - Brett Smith ${ }^{7}$. Erik Starman $^{7} \cdot$ Stacey R. Sueoka $^{7}$ - Richard T. Summers ${ }^{1}$ - Aimee Szabo ${ }^{7}$. Louis Szabo $^{7}$ - Stephen B. Wampler ${ }^{1}$. Timothy R. Williams ${ }^{7}$. Charles White ${ }^{7}$

Received: 21 August 2020 / Accepted: 10 November 2020 / Published online: 4 December 2020 (C) The Author(s) 2020

\footnotetext{
This article belongs to the Topical Collection: The Daniel K. Inouye Solar Telescope (DKIST) Guest Editors: Thomas R. Rimmele, Philip R. Goode, Michael Knölker, Jeffrey R. Kuhn, and Robert Rosner
}

Electronic supplementary material The online version of this article (https://doi.org/10.1007/s11207-020-01736-7) contains supplementary material, which is available to authorized users.

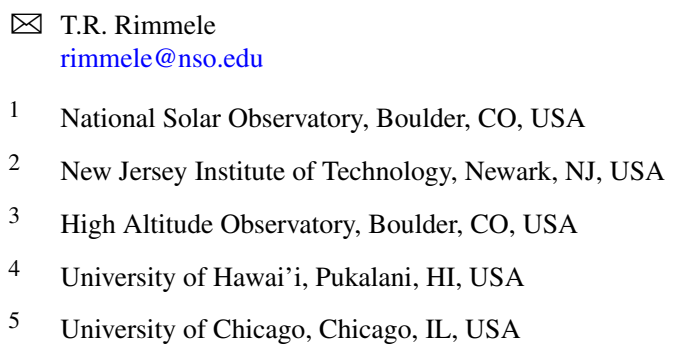


Abstract We present an overview of the National Science Foundation's Daniel K. Inouye Solar Telescope (DKIST), its instruments, and support facilities. The $4 \mathrm{~m}$ aperture DKIST provides the highest-resolution observations of the Sun ever achieved. The large aperture of DKIST combined with state-of-the-art instrumentation provide the sensitivity to measure the vector magnetic field in the chromosphere and in the faint corona, i.e. for the first time with DKIST we will be able to measure and study the most important free-energy source in the outer solar atmosphere - the coronal magnetic field. Over its operational lifetime DKIST will advance our knowledge of fundamental astronomical processes, including highly dynamic solar eruptions that are at the source of space-weather events that impact our technological society. Design and construction of DKIST took over two decades. DKIST implements a fast (f/2), off-axis Gregorian optical design. The maximum available field-of-view is 5 arcmin. A complex thermal-control system was implemented in order to remove at prime focus the majority of the $13 \mathrm{~kW}$ collected by the primary mirror and to keep optical surfaces and structures at ambient temperature, thus avoiding self-induced local seeing. A high-order adaptive-optics system with 1600 actuators corrects atmospheric seeing enabling diffraction limited imaging and spectroscopy. Five instruments, four of which are polarimeters, provide powerful diagnostic capability over a broad wavelength range covering the visible, near-infrared, and mid-infrared spectrum. New polarization-calibration strategies were developed to achieve the stringent polarization accuracy requirement of $5 \times 10^{-4}$. Instruments can be combined and operated simultaneously in order to obtain a maximum of observational information. Observing time on DKIST is allocated through an open, merit-based proposal process. DKIST will be operated primarily in "service mode" and is expected to on average produce $3 \mathrm{~PB}$ of raw data per year. A newly developed data center located at the NSO Headquarters in Boulder will initially serve fully calibrated data to the international users community. Higher-level data products, such as physical parameters obtained from inversions of spectro-polarimetric data will be added as resources allow.

Keywords Telescopes · Instrumentation · Sun · Magnetic fields · Photosphere · Chromosphere · Corona

\section{Introduction}

The US National Science Foundation's (NSF) $4 \mathrm{~m}$ Daniel K. Inouye Solar Telescope (DKIST) on Haleakalā, Maui, Hawai'i is the largest optical solar telescope in the world. DKIST delivers spatial resolution and sensitivity that enable astronomers to unravel many of the mysteries that the Sun presents, including the origin of solar magnetism, the mechanisms of coronal heating and drivers of the solar wind, flares, coronal mass ejections, and variability in solar output.

The state-of-the-art adaptive-optics system provides diffraction-limited imaging and the ability to resolve features approximately $20 \mathrm{~km}$ on the Sun. Achieving this unprecedented spatial resolution is critical for the ability to observe solar magnetic fields (Stenflo, 1994;

6 Leibniz Institute for Solar Physics (KIS), Freiburg, Germany

7 National Solar Observatory, Makawao, HI, USA

8 University of Colorado, Boulder, CO, USA

9 Astrophysics Research Centre, School of Mathematics and Physics, Queen's University Belfast, Belfast BT7 1NN, Northern Ireland, UK 
Wiegelmann, Thalmann, and Solanki, 2014) at small spatial scales. Fundamental processes such as the emergence and destruction of magnetic flux, the heating processes and wave generation, and the fundamental turbulent processes that occur on small spatial scales (de Wijn et al., 2009). It has long been argued that the critical scales are the photon mean-free path and the pressure scale height (Stenflo, 2008). To resolve both fundamental length scales in the deepest accessible layers of the solar atmosphere with near-infrared observations, a resolution of $70 \mathrm{~km}$ or $0.1 \mathrm{arcsec}$ is required. The deepest layers of the photosphere can be observed at a wavelength of 1.6 micron where the opacity of the solar atmosphere has a minimum (Avrett, 2003). The sensitivity of magnetic-field measurements using the Zeeman effect increases at longer wavelength due to the wavelength dependence of the Zeeman splitting of spectral lines. The requirement to resolve $0.1 \mathrm{arcsec}$ or better at a wavelength of 1.6 microns is equivalent to the requirement for a minimum aperture of $4 \mathrm{~m}$. Resolving spatial scales on the order of a few tens of kilometers is of crucial importance to be able to test physical models and thus understand how the physics of the small scales ties into the larger-scale problems. Some fundamental physical processes occur at scales that remain unresolved even with DKIST (Stenflo, Keller, and Gandorfer, 1998; Bianda, Stenflo, and Solanki, 1998; Stenflo, 2000, 2013). Spatially resolved observations using the Hanle effect are an equally important diagnostic tool. Spatially unresolved Hanle measurements benefit immensely from the high photon-flux provided by DKIST's $4 \mathrm{~m}$ aperture.

Measurements of the solar magnetic field of sufficient resolution and sensitivity and throughout the solar atmosphere, including the corona, are key to solving many of the outstanding problems of solar astronomy. Five instruments were defined at the outset of the project in collaboration with the Science Working Group (SWG). Four of these instruments will provide highly sensitive measurements of solar magnetic fields. It is anticipated that additional instrumentation will be added during the lifetime of DKIST. The design allows DKIST to operate as a coronagraph at infrared wavelengths where the sky background is low and bright coronal emission lines are available (Kuhn, Penn, and Mann, 1996; Judge et al., 2002; Lin, Kuhn, and Coulter, 2004; Dima, Kuhn, and Schad, 2019). Mapping of the coronal magnetic field is a key objective of DKIST, which was designed to address basic research aspects of space weather and help improve predictive capabilities. During its nominal lifetime of two magnetic solar cycles DKIST will provide observational data that will allow scientists to advance and refine models and simulations of solar phenomena. DKIST science operations are entirely driven by community proposals solicited through a regular proposal cycle.

DKIST replaces the $1 \mathrm{~m}$ class telescopes of the National Solar Observatory with a $4 \mathrm{~m}$ class solar telescope. While DKIST is the first new telescope in its class, similar telescopes are being pursued by the international community. The European Solar Telescope (EST) will be sited on the La Palma, Canary Islands, Spain (Collados et al., 2013). The science mission of the $4 \mathrm{~m}$ EST is summarized by Schlichenmaier et al. (2019). Once operational, the EST will be able to observe the Sun with a time difference of 11 hours from DKIST. When operated in coordination, DKIST and EST will provide extended temporal coverage that would, for example, enable tracking of an active region in order to study its evolution and enhance the probability to capture observations of flares. Other projects include the Russian 3 m-aperture Large Solar Telescope planned to be located in the Sayan Solar Observatory at an altitude of more than $2000 \mathrm{~m}$ (Grigoryev et al., 2020), India's National Large Solar Telescope with aperture $2.5 \mathrm{~m}$ (Hasan, 2012), and the Chinese Giant Solar Telescope with a planned aperture of $8 \mathrm{~m}$ (Deng et al., 2016). It is conceivable that a network of largeaperture, ground-based solar telescopes could eventually be implemented and provide close to 24 hour temporal coverage of observations of the highest resolution. 
Early concept development and mission definition for DKIST began at the National Solar Observatory (NSO) in the mid 1990s with the Coronagraphic Low Emissivity Astronomical Reflector (CLEAR) effort (Beckers, 1995; Beckers and NSO Staff, 1997). At the same time development of key technologies, such as adaptive optics (AO) (Rimmele and Radick, 1998; Rimmele, 2000) and infrared instrumentation and methodology progressed (Penn and Kuhn, 1994; Kuhn et al., 1999; Lin, Penn, and Tomczyk, 2000). A key milestone was the high ranking that the Advanced Solar Telescope (AST) project received from the 2000 decadal review (National Research Council Astronomy and Astrophysics Survey Committee, 2000). Subsequent decadal reviews reaffirmed the national priority of what became the Advanced Technology Solar Telescope (ATST). Between 2003 and 2008 the project engaged in an NSF funded design and development phase. Major trade studies were conducted and subsequent design decisions were made early on including the off-axis Gregorian optical design, the f-number and off-axis distance of the primary mirror, choice of telescope mount (altitude-azimuth (alt-az)), enclosure type and instrument lab with rotating instrument platform (Rimmele et al., 2003b; Oschmann et al., 2004). Early concept and design studies also benefited from the work performed previously by the Large Earth-based Solar Telescope (LEST: Engvold, 1991).

An extensive site survey was conducted between 2000 and 2005 (Hill et al., 2004, 2006). The ATST Site Survey Working Group oversaw the development and construction of instrumentation to measure daytime solar seeing (Beckers, 2002), sky brightness (Lin and Penn, 2004), clear time fraction, dust levels, and water-vapor content. These quantities were measured at six candidate sites, which had been selected from an initial list of 72 candidates. The six tested sites represent a cross-section of geographical locales: continental mountain (Sacramento Peak, New Mexico), continental mountain lake (Panguitch Lake, Utah), peninsula mountain (San Pedro-Martir, Baja California, Mexico), coastal mountain lake (Big Bear Lake, California), Atlantic island mountain (La Palma, Canary Islands, Spain), and Pacific island mountain (Haleakala, Maui, Hawai'i). Based on the site-survey results Haleakalā was recommended by the Science Working Group and formally approved as the ATST site by AURA and NSF. Haleakalā provides the unique combination of excellent seeing and low sky brightness, i.e. the site characteristics needed to support both the high-resolution and the coronal science objectives. Conceptual, preliminary, and final design phases and reviews were conducted between 2003 and 2009 (Keil, Rimmele, and Keller, 2003; Rimmele et al., 2003a,b, 2005; Keil et al., 2003, 2004; Oschmann et al., 2004; Wagner et al., 2006; Rimmele, Keil, and Wagner, 2008; Keil, Rimmele, and Wagner, 2010). Design and development of first-light instrumentation progressed simultaneously (Rimmele et al., 2004; Elmore et al., 2014).

After successfully passing its final design review, the project was approved for construction, the construction funds were awarded by NSF in 2010, and the project's construction phase commenced (Rimmele et al., 2010, 2012; McMullin et al., 2012; Warner et al., 2013). The site-permitting process was concluded and construction activities at the Haleakalā site began in December 2012. With the start of site construction, the ATST was renamed the Daniel K. Inouye Solar Telescope in 2013. The construction effort progressed over a period of seven years (McMullin et al., 2014, 2016; Rimmele et al., 2014; Warner et al., 2018, 2020) and achieved first solar light in December 2019. Integration and commissioning of first-light instruments and instrument supporting systems is progressing through 2020 (Elmore et al., 2014). Construction of a base facility located in Pukalani was completed in 2018. The base facility provides office and laboratory space and includes a remote operations room from which observations of DKIST can be monitored, guided, and, in the near future, conducted. The first proposal call for the operations commissioning phase (OCP) was released in May 2020. 
The scientific mission of DKIST is relevant far beyond solar astronomy. Magnetic fields are ubiquitous throughout the universe and play a key role in, for example, early universe galaxy formation (Parker, 2000; de Gouveia Dal Pino, 2011; Soida et al., 2012). Due to its proximity, the Sun provides us with a laboratory where fundamental physical processes that operate throughout the universe can be studied in detail that no other astronomical object affords us. The advances in our understanding of basic physical processes that DKIST provides will inform and help advance astronomy and other related fields, such as plasma physics.

This article summarizes the design and capabilities of DKIST as well as its science goals and operational aspects, and it is the first in a Topical Collection on DKIST. First results are presented in Section 14. Science goals, instrument capabilities, and operational aspects are discussed very briefly and are presented in significant detail in separate articles. The instruments are described in the following articles: Visible Broadband Imager (VBI: Wöger et al., 2021), Visible Spectro-Polarimeter (ViSP: de Wijn et al., 2021), Diffraction-Limited NearInfrared Spectro-Polarimeter (DL-NIRSP: Jaeggli et al., 2021), Cryogenic Near-Infrared Spectro-Polarimeter (CRYO-NIRSP: Fehlmann et al., 2021), and Visible Tunable Filter (VTF: von der Lühe et al., 2021). Methodology and first results of the all-important polarimetric calibration of the telescope and instrument optical system are summarized by Harrington et al. (2021). Included in the Topical Collection are an extensive review of the critical science to be performed with DKIST (Rast et al., 2021), descriptions of DKIST operations (Tritschler et al., 2021), and the DKIST Data Center (Davey et al., 2021). Finally, this article provides a comprehensive, but necessarily incomplete, list of references to individual articles and documents published over the course of the two-decade-long project. These publications describe design and implementation details of various systems and subsystems.

\section{DKIST Science Drivers}

The science goals of DKIST (formerly ATST) were summarized early on in several publications (Rimmele et al., 2001, 2003b; Keil et al., 2004; Rimmele and ATST Team, 2008; Berger et al., 2014; Rast, Cauzzi, and Martínez Pillet, 2019) and documents (Rimmele and the ATST Science Working Group, 2005). The field of solar astronomy has evolved since the definition phase of DKIST. Significant progress has been made, both observationally and with theoretical modeling. It is beyond the scope of this article to provide a comprehensive summary of science drivers and goals of DKIST from today's perspective. Instead such summary of the broad and diverse spectrum of science objectives that DKIST will pursue during the first few years of operations is presented in a separate contribution to this Topical Collection entitled Critical Science Plan for the DKIST (Rast et al., 2021).

A main driver for the $4 \mathrm{~m}$ aperture of DKIST continues to be the requirement to spatially resolve the fundamental astrophysical processes at their fundamental scales throughout the solar atmosphere (e.g. Parker, 2000). Ever-improving numerical simulations (Schüssler and Vögler, 2006; Stein and Nordlund, 2006, 2012; Rempel and Schlichenmaier, 2011; Stein, 2012; Rempel, 2012, 2020) have reached grid resolution of a few tens of kilometers and predict that crucial physical processes occur on spatial scales of tens of kilometers. However, any model and model assumptions must be tested and verified with observations. Progress towards the required spatial resolution has been made with observational capabilities as well. While the resolution of the Hinode/Solar Optical Telescope (Tsuneta et al., 2008) is limited by its $50 \mathrm{~cm}$ aperture, consistent resolution and image quality can be achieved over long periods of time, and this space mission has advanced our understanding of the 
Sun significantly. Meter-class ground-based solar telescopes, including the Swedish Solar Telescope on La Palma, the Dunn Solar Telescope at Sacramento Peak, NM, and the Vacuum Tower Telescope on Tenerife, have been the "work horses" of solar astronomy and drivers of innovation for decades. Over the last decade, new $1.5 \mathrm{~m}$ class telescopes were built, namely the $1.6 \mathrm{~m}$ aperture Goode Solar Telescope (GST) at Big Bear Solar Observatory (Goode and Cao, 2012a,b) and the $1.5 \mathrm{~m}$ GREGOR on Tenerife (Schmidt et al., 2012). In particular, the $1.6 \mathrm{~m}$ off-axis GST served as a pathfinder for optics-technology development.

In order to achieve spatial resolution that can match the resolution of modern MHD simulations, the $4 \mathrm{~m}$ aperture DKIST operating at the diffraction limit is required. Resolving features at these scales is of the utmost importance to be able to test various models and simulations. The close interaction between model and observation in an iterative process is key to advancing our understanding of the underlying physics. Only through this interaction can we hope to solve long-standing questions. An example is the weak, small-scale magnetic fields that cover the entire solar surface (Lin and Rimmele, 1999; Lites, 2002, 2009; Lites et al., 2008, 2017; Pietarila Graham, Danilovic, and Schüssler, 2009; Bellot Rubio and Orozco Suárez, 2019) and in what way these weak fields might drive large-scale phenomena such as chromospheric and coronal heating. Emerging small-scale fields may lead to reconnection events that contribute to coronal heating and the acceleration of the solar wind (Isobe, Proctor, and Weiss, 2008). A large fraction of the solar magnetic flux is likely contained in these weak fields and their importance for the solar dynamo or dynamos is also not yet understood (Schüssler, 2005; Cameron and Schüssler, 2015). Using the increased magnetic sensitivity of infrared lines at 1.56 microns, which originate deep in the photosphere, in addition to the spectral diagnostic available in the visible, DKIST will be able to test MHD models of surface dynamo action (Rempel, 2018, 2020). The $4 \mathrm{~m}$ aperture provides sufficient resolution $(0.08 \mathrm{arcsec})$ at these near infrared wavelengths.

Similarly, modeling and numerical simulations of chromospheric (Bjørgen et al., 2019) and coronal magnetic-field structures (Rempel, 2017) have advanced continuously and significantly during recent years. Examples include modeling of flares (Cheung et al., 2019) and coronal mass ejections (CMEs) (Fan and Gibson, 2004; Gibson and Fan, 2006; Fan, Gibson, and Tomczyk, 2018). At the same time, chromospheric and, in particular, coronal magnetic-field measurements continue to suffer from insufficient spatial resolution and sensitivity. Measurements of the coronal magnetic field, in particular the Stokes- $V$ component, have not progressed significantly since the pioneering, proof-of-concept demonstration with Solar- $C$, a $46 \mathrm{~cm}$ off-axis mirror coronagraph on Haleakalā, by Lin, Penn, and Tomczyk (2000) and Lin, Kuhn, and Coulter (2004). Observations of the faint corona are inherently photon-starved. The coronal intensity is of order $10^{-6}$ of the disk intensity. The expected polarimetric signals are of order $10^{-3}$ or $10^{-4}$ of the coronal intensity. Hence, providing a large photon collecting area is an equally strong driver for the $4 \mathrm{~m}$ aperture, which will provide a collecting area 75 times that of Solar-C. We note that, for coronal magnetometry, DKIST will not attempt to observe at the diffraction limit but instead will be used as a light-bucket. With spatial resolution of one to two arcsec, sensitivity of order $10 \mathrm{G}$ or less can be achieved with a temporal resolution of tens of seconds to minutes. Special care was taken with the DKIST design to avoid scattered light. The off-axis design avoids spider diffraction. In-situ cleaning and washing are provided to mitigate scattered light from dust contamination. Finally, DKIST is located at an excellent coronal site (Hill et al., 2006).

The solar atmosphere is highly structured by the magnetic field, which permeates the solar atmosphere from photosphere to the corona (see, e.g., Wedemeyer-Böhm, Lagg, and Nordlund, 2009, Figure 16). Understanding the three-dimensional (3D) structure of the solar magnetic field and how magnetic fields connect throughout the solar atmosphere is therefore 
of particular interest and a prerequisite for solving major scientific problems such as flares, coronal mass ejections, and coronal heating. DKIST's instrumentation is designed to provide simultaneous multi-line polarimetry at visible and infrared wavelengths in a coordinated fashion and sample the magnetic field at different heights in the solar atmosphere. Such measurements provide crucial information about the 3D-structure of the magnetic field. In addition, coordination with other ground-based and space-based telescopes has been taken into account while planning the operations of DKIST. Using DKIST as part of an expanded observing system, sometimes referred to as multi-messenger astronomy, can greatly enhance the diagnostic power of each individual entity (Martínez Pillet et al., 2020).

The Critical Science Plan (CSP: Rast et al., 2021) captures in great detail and with many concrete examples the challenges of solar physics from today's perspective. As an NSFfunded national facility, DKIST operations are entirely proposal driven. The CSP effort aimed to assist the community to get prepared for key observations with DKIST. Led by the Science Working Group and with assistance from the project science team, the community developed a large set of Science Use Cases or pre-proposals, designed for the "as-built" DKIST facility. It is clear from the CSP that the need for DKIST with its large aperture and powerful set of instruments has only increased during the time it took to construct the facility. Achieving the many and broad science goals, which are expected to be accomplished over a period of 44 years (two solar magnetic cycles), requires the flexible and versatile design of DKIST that supports diffraction-limited observing at visible and near- and farinfrared wavelengths, and coronal observations near the limb of the Sun. The CSP outlines initial observing goals to be implemented during the first few years of DKIST operations.

\subsection{Science Requirements Definition}

The definition phase for a science facility that can serve a broad science community over a period of decades was a significant challenge. The telescope and its initial instrumentation suite had to be designed with sufficient flexibility to accommodate future growth, and to allow for adaptation to evolving science questions. In that sense, the design considerations and drivers were quite different compared a space mission with limited (a few years) mission duration and a specific and limited set of science objectives. The Science Working Group in the early 2000s developed the Science Requirements Document (SRD: Rimmele and the ATST Science Working Group, 2005), which, along with the Operational Concepts Document (OCD: Trischler, 2020), constitutes the set of top-level specification documents that have guided the design and construction of DKIST. The process of top-level specification development involved a large section of the community developing observing or science use-cases. The strategy was to distill the most stringent technical requirements for telescope and instruments from the most demanding observational requirements, as envisioned at the time, and derived from the set of all use-cases. Both use-cases and requirements were captured in the SRD. Examples of requirements include field-of-view, image quality, allowable scattered light, and polarimetric sensitivity and accuracy. A subset of requirements is summarized in Table 1.

The reasons for the $4 \mathrm{~m}$ aperture were discussed in Sections 1 and 2, and the references therein. We note that an even larger aperture was discussed by the SWG in the context of far-infrared observations. For example, magnetic-field measurements at 12 microns are of interest to explore the magnetic-field structure of the upper photosphere near the temperature minimum (Moran et al., 2000; Jennings et al., 2002). An aperture of $24 \mathrm{~m}$ diameter would be required to achieve a resolution of 0.1 arcsec at this wavelength, a goal that will likely have to be achieved by a future solar interferometer. The SWG determined the $4 \mathrm{~m}$ aperture to be 
Table 1 Summary of DKIST capabilities.

\begin{tabular}{ll}
\hline Aperture & 4 m unobstructed \\
Optical Configuration & Gregorian $\mathrm{f} / 2$ \\
Mount & altitude-azimuth mount \\
Field of View & up to 5 arcmin, 2.8 arcmin AO instruments \\
Adaptive Optics & conventional, MCAO upgrade option \\
Adaptive Optics Strehl (S) & $\mathrm{S}(500 \mathrm{~nm})>0.3$ for $\mathrm{r}_{0}>7 \mathrm{~cm}$ \\
& $\mathrm{~S}\left(630 \mathrm{~nm}>0.6\right.$ for $\mathrm{r}_{0}(630 \mathrm{~nm})>20 \mathrm{~cm}$ \\
Resolution & diffraction limited, $0.026^{\prime \prime}(20 \mathrm{~km})$ @ $500 \mathrm{~nm}$ \\
Polarization accuracy & $5 \times 10^{-4}$ \\
Polarization Sensitivity & photon limited \\
Coronagraph & at IR wavelengths \\
Scattered light control & in-situ CO 2 cleaning and mirror washing \\
Instruments & five $(5)$ facility class instruments \\
Polarimeters & four $(4)$ polarimeters, UV to IR coverage \\
Spectral range coudé instruments & 380 nm -5000 nm \\
Operations & multi-instrument experiments \\
& coordinated with ground- and space based facilities \\
Lifetime & service and traditional PI mode \\
\hline
\end{tabular}

necessary and sufficient to achieve the vast majority of science goals. The maximum field of view (FOV) provided by the telescope to the instruments is 5 arcmin, which allows off-limb observations of, for example, large coronal-loop systems and prominences. The maximum FOV for AO-corrected observations is 2.8 arcmin circular, i.e. a FOV that covers a large active region. The conventional AO system provides optimal correction within the isoplanatic patch. However, during the day ground-layer turbulence dominates and some level of correction is provided over a larger FOV by the AO system (Rimmele and Marino, 2011). An all-reflective optical design was chosen to allow wavelength coverage from the UV to far-infrared. The performance requirements on the AO system as quantified by the delivered Strehl-ratio were specified as a function of seeing conditions. The Strehl ratio is the ratio of energy in the diffraction-limited core vs. energy in the seeing-limited halo of the point spread function (PSF). For excellent seeing conditions $\left(\mathrm{r}_{0}(630 \mathrm{~nm})>20 \mathrm{~cm}\right)$ the AO is required to deliver $\mathrm{S}=0.6$. This requirement was derived with the help of MHD simulations of small-scale, mixed-polarity fields (Stein and Nordlund, 2006). The simulated images and maps of physical parameters (e.g. Stokes- $V$ ) were convolved with AO-corrected PSFs of varying Strehl ratio in order to produce simulated observations, which in turn guided the development of Strehl-ratio requirements (Rimmele and the ATST Science Working Group, 2005). Specifying the Strehl at one wavelength determines what Strehl will be achieved at other wavelengths. For example, $S=0.6$ for $r_{0}(630 \mathrm{~nm})=20 \mathrm{~cm}$ translates to $S=0.92$ at 1.6 microns for the same seeing conditions. The requirement for polarization accuracy of $5 \times 10^{-4}$ is particularly challenging and is discussed in Section 6 . The anticipated operational lifetime of DKIST covers at least two Hale cycles, which imposes many stringent requirements on mechanical and optical systems. In order to minimize scattered light produced by dust particles and other contaminates that collect on the surface of the primary mirror, the capability to clean the mirror in situ with either $\mathrm{CO}_{2}$ snow or by washing was deigned in. Finally, it is the instruments that provide the science data. The decision to pro- 
vide a suite of facility-class instruments at the start of operations that enable the community to perform the critical measurements, in particular measurements of the $3 \mathrm{D}$ magnetic-field structure, was made at the very beginning of the construction project.

Future upgrade options such as multi-conjugate adaptive optics (MCAO) to be implemented at a later time were also considered at the time as those might inform design decisions. Operational concepts were subsequently developed. Tritschler et al. (2016) and Tritschler et al. (2021) describe concepts such as service mode, coordinated observations, target-of-opportunity observations, and user interactions with the facility and its instruments. SRD and OCD and the individual Instrument Science Requirements Documents (ISRDs) constitute the set of top-level specification documents from which subsequent requirements flow.

A rigorous systems-engineering approach was applied for the telescope facility and instruments with flow down of top-level science requirements to subsequent systems, subsystems and component engineering requirements (Oschmann, 2004; Craig et al., 2014; Hubbard, Craig, and Kneale, 2016). The systems-engineering processes followed the standard V-Diagram approach ensuring adequate component, subsystem, and system testing and verification at all stages of the project. Systems engineering also included development of imaging and wavefront-error budgets and active tracking and management of error budgets throughout the process. A new approach to polarimetry system error budgets was developed and is described in a series of publications (Harrington and Sueoka, 2017; Harrington et al., 2017, 2021; Harrington and Sueoka, 2018a,b; Harrington, Sueoka, and White, 2019). The process concludes with final verification of SRD, OCD, and ISRD requirements and hand-over of the facility to operations.

\section{DKIST Design Overview}

Figure 1 shows a rendering of the DKIST facility and its main subsystems. Figure 2 displays a recent image of the completed DKIST at the Haleakala site. The main telescope is a $4 \mathrm{~m}$ off-axis Gregorian design (Rimmele et al., 2003b). The optics is supported by the alt-az telescope mount structure (Warner et al., 2006; Jeffers et al., 2012b,c), which provides accurate and precise pointing and tracking (Dreyer et al., 2014). The telescope is protected from the environment by a thermally controlled and well-ventilated enclosure (Murga et al., 2011, 2012). A number of optical elements relay the light beam to the environmentally controlled coudé lab (Phelps et al., 2010) where all first-light instruments are located. The instruments are installed on a rotating platform (Jeffers et al., 2016), which also serves as image de-rotator and allows for alignment of the spectrograph slits, for example, parallel or perpendicular to the limb. The tip-tilt mirror and the deformable mirror of the conventional high-order AO system (Rimmele et al., 2006; Richards et al., 2010; Johnson et al., 2018) are integrated into the transfer optics. The AO-corrected light beam feeds the four instruments that are designed to perform diffraction-limited observations at visible and near-infrared wavelengths, and which can operate simultaneously. Facility buildings include a supportand-operations building, the telescope pier (Jeffers et al., 2012a), and the lower enclosure (Phelps and Warner, 2008), and a detached utility building. The utility building houses mechanical equipment, such as chillers, pumps, air-handling units, and electrical equipment. The utility building is part of facilities thermal systems (Phelps et al., 2012). The supportand-operations building has three main floor levels with the telescope control room and an instrument lab at the same level as a rotating coudé instrument clean-room inside the telescope pier (Phelps et al., 2010). A separate instrument-preparation laboratory provides a second clean-room environment that is used to assemble and test instruments or instrument and telescope optical components. 


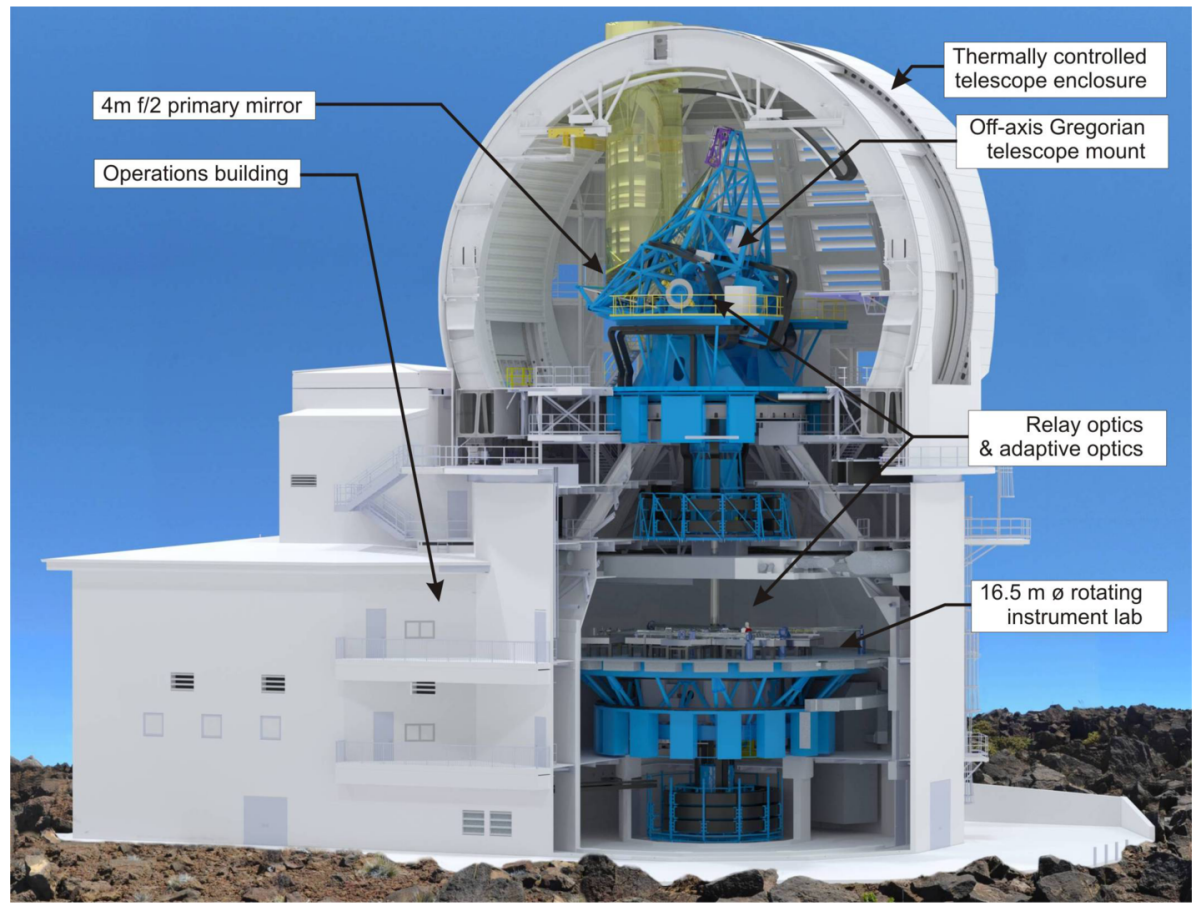

Figure 1 Schematic of DKIST facility and its major components. The overall facility height is $41.4 \mathrm{~m}$. The diameter of the rotating instrument platform is $16.5 \mathrm{~m}$.

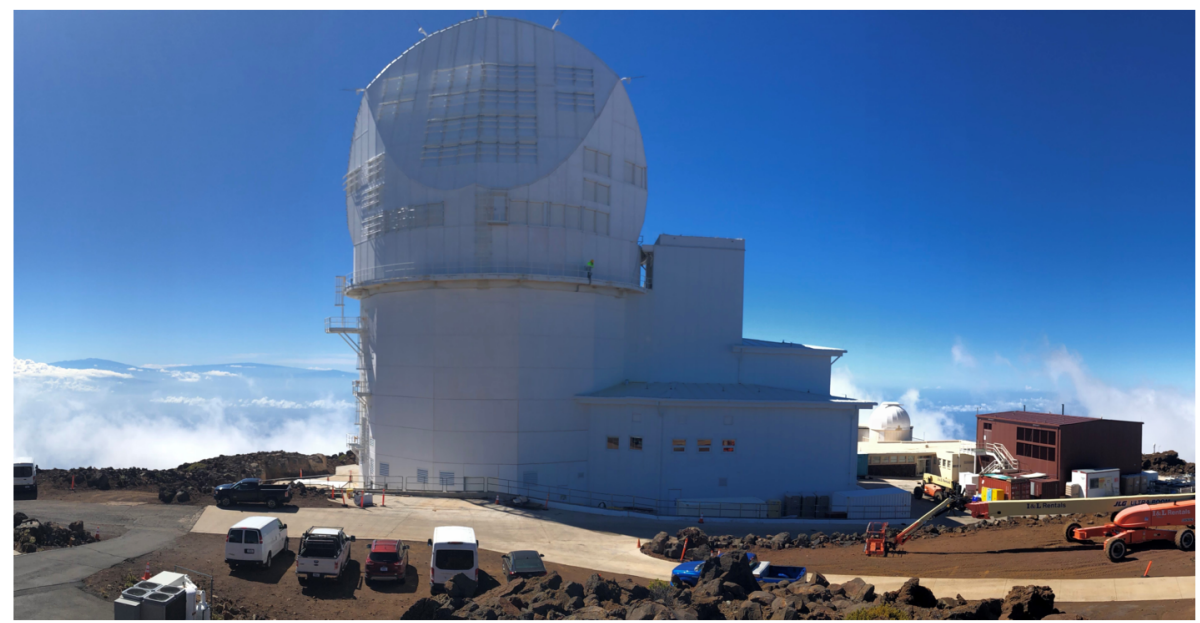

Figure 2 The outside of the completed DKIST on Haleakalā. The telescope building with lower and upper enclosure and the attached Support and Operations building, including platform lift used to transport M1 to the telescope level, are shown on the right of the image. Vent gates ensure sufficient air flow through the enclosure during operations. The utility building, which supplies coolant to the main facility and houses backup power systems is visible to the right of the main facility. The overall facility height is $41.4 \mathrm{~m}$. 


\section{DKIST Optical System Design}

To achieve the telescope scattered-light performance required for sensitive coronal magnetometry, DKIST uses an unobstructed, fast, off-axis optical configuration that recent solar telescopes have adopted (Kuhn et al., 2003; Goode and Cao, 2012a). Aspects of the optical design of DKIST evolved from an early design presented by Rimmele et al. (2003b) and Hansen, Price, and Hubbard (2006). While the design of the main telescope (i.e. the primary (M1) and secondary (M2) system) has remained unchanged, the design of transfer optics and, in particular, the coudé light-distribution system did undergo significant changes as instrument concepts and the AO design evolved. For example, initial concepts located the coronal instrument CRYO-NIRSP at the Nasmyth focal station. The deformable mirror (DM) was located at the telescope level (i.e. outside-air temperatures) at the position of M5. The thermally stable and contamination-controlled environment, as well as the stable gravity vector provided by the coudé instrument lab drove towards locating all instruments and the DM at the coudé lab. However, a Nasmyth station remains an option for future additional instrumentation.

A schematic of the final design is shown in Figure 3. In the following we describe in some detail groups of optical elements and their functionality, namely:

i) The main telescope consisting of primary and secondary mirrors, a heat-stop, and a Lyot-stop. Integrated into the main telescope is the Gregorian Optical Station (GOS).

ii) The transfer optics, which direct the light to the coudé lab.

iii) The coudé optics, which provide a collimated beam and an image of the entrance aperture for the DM.

iv) The facility instrument-distribution optics (FIDO), which in a reconfigurable manner distribute light to the various instruments.

We note that M1 is coated with unprotected aluminum at the coating facility located on Haleakalā at the Air Force Maui Optical Station (AMOS). Figure 4 shows the M1 after it received its first aluminum coating at the AMOS facility. We note that the procedure to recoat the M1 is a major undertaking that requires several weeks of telescope down time. The required frequency for re-coating the M1 will be established by monitoring the scattered light performance during operations and in part is determined by the effectiveness of the M1 cleaning procedures. All other mirrors are coated with protected silver in order to achieve high transmission of the optical system.

\subsection{Main Telescope}

The main telescope is a $4 \mathrm{~m}$ off-axis Gregorian with a fast $\mathrm{f} / 2$ primary mirror (M1) and a $0.65 \mathrm{~m}$ concave ellipsoid (conic constant $=-0.54$ ) secondary $(\mathrm{M} 2)$. A heat stop at the prime focus passes a 5 arcmin science FOV and rejects most of the $13 \mathrm{~kW}$ solar heat load, making thermal control of subsequent optical components a manageable problem. M1 is located $28 \mathrm{~m}$ above ground in order to get above the ground seeing that builds up during the day. Using the site-survey data (Hill et al., 2006), a trade-off study was performed to maximize the number of good and excellent seeing hours (i.e. maximize science productivity) while balancing with feasibility, cost, and other considerations, such as visibility of the facility from major population centers.

The scientific and technical drivers for the off-axis design are discussed by Rimmele et al. (2003a) and are briefly summarized as follows. The unobstructed aperture provides the full $12.56 \mathrm{~m}^{2}$ collecting area and is free from the diffraction effects of the secondary 
Figure 3 Optical design of DKIST. The main optical groups including the main telescope, with the $4 \mathrm{~m}$ off-axis primary and secondary mirrors, and the transfer and coudé optics are shown.
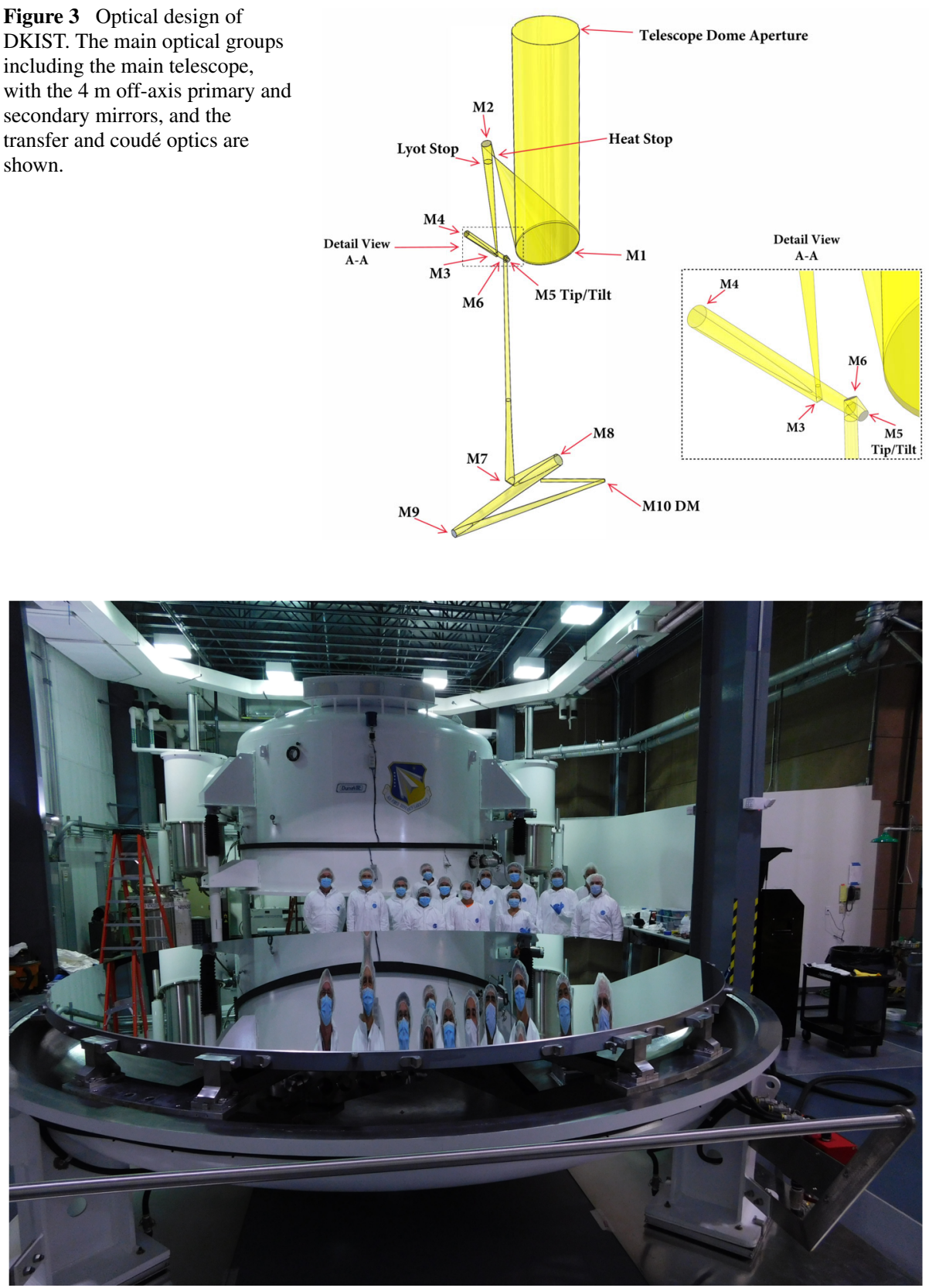

Figure 4 The 4-meter primary mirror (M1) after coating at the AMOS coating facility on Haleakalā.

mirror and its support spider. This is important not only for the coronal (low scattered light) science objectives but also simplifies the design, operations, and performance of the solar AO system, which of course is crucial for obtaining diffraction-limited observations. The off-axis location of the secondary mirror provides physical space to implement the heat stop 
at prime focus and the required cooling lines. The secondary mirror can also be sheltered from wind buffeting and supported in a more robust way than is possible in an on-axis configuration, thereby mitigating potential image-jitter problems.

In order to realize the advantages of the off-axis approach, a number of technology challenges had to be overcome. For example, the DKIST M1 asphericity and alignment requirements are more stringent than earlier off-axis telescopes because of the larger off-axis angle. The manufacturing of the $4.24 \mathrm{~m}$ thin meniscus primary mirror with a clear aperture of $4 \mathrm{~m}$ and a thickness of only $75 \mathrm{~mm}$ (driven by thermal-control requirements) posed challenges and risk. The primary mirror is a concave paraboloid (conic constant $=-1$ ), which can be pictured as an off-axis segment of a $12 \mathrm{~m}$ parent parabolic mirror with an offaxis offset of $4 \mathrm{~m}$ (Hubbard and Oschmann (2004), Figure 3). Specifications for the surface quality, including the surface-figure errors $(<25 \mathrm{~nm} \mathrm{rms}$ and $<8 \mathrm{~nm}$ rms total for spatial periods from $1 \mathrm{~mm}$ to $100 \mathrm{~mm}$ ), surface roughness (1.2 $\mathrm{nm}$ achieved) and Bi-Directional Reflectance Distribution Function (BRDF), were challenging due the fact that DKIST operates at visible wavelengths including the very blue end of the spectrum. The BRDF requirement (Tayabaly, 2013) is driven by the coronal-science objectives. The Zerodur mirror blank was produced by Schott AG (Jedamzik, Werner, and Westerhoff, 2014). The polishing was completed at the College of Optical Sciences at the University of Arizona and met or exceeded all requirements to produce "the smoothest large mirror ever made" (Oh et al., 2016). The detailed fabrication process and metrology included the grinding to finish, implementing an extremely stable hydraulic support, IR and visible deflectometry, interferometry, and computer-controlled fabrication process. We note that the BBSO $1.6 \mathrm{~m}$ off-axis primary mirror served as path finder for development of polishing and metrology methodology development.

M1 is actively supported by 118 axial actuators and 24 lateral supports (Cho, Price, and Moon, 2006). The active M1 support system, including the thermal control of M1 (Hansen, Bulau, and Phelps, 2008) and a cooled $4 \mathrm{~m}$ aperture stop, were produced by Advanced Mechanical and Optical Systems (AMOS) in Belgium. The primary-mirror support system was optimized for the telescope at low-elevation pointing to minimize gravity and thermal effects during the morning hours when seeing conditions are optimal. It is interesting to note that at near-horizon pointing M1 is actually suspended from its axial actuators. Due to the requirement to minimize scattered light, the primary-mirror assembly and special equipment were designed and built to allow for frequent $\mathrm{CO}_{2}$ cleaning and in-situ washing. $\mathrm{M} 2$ is made of a light-weight $\mathrm{SiC}$ structure and serves as fast steering mirror. M2 was manufactured by L3 Brashear, Integrated Optical Systems, Inc. A thermal-control system maintains the front-surface temperature of $\mathrm{M} 2$ within $\pm 1{ }^{\circ} \mathrm{C}$ of ambient via air-jet impingement on its back surface. $\mathrm{SiC}$ was chosen based on its superb thermal characteristics and stiffness superiority as determined through extensive parametric trade-off studies (Cho, DeVries, and Hansen, 2007). The off-axis M2 produces an $\mathrm{f} / 13$ beam that provides a secondary image at the Gregorian Optical Station (GOS). The tip-tilt M2 provides image-motion compensation for near-limb coronal observations and future Nasmyth instruments. The M2 mechanism is mounted on a six-degree-of-freedom hexapod allowing M2 to be part of an active-alignment strategy.

Figure 5 displays the main telescope during first solar light that was achieved in December 2019. The M1 and the $4 \mathrm{~m}$ diameter aperture stop are illuminated with sunlight. The $\mathrm{f} / 2$ M1 forms an image of the solar disk in prime focus where the heat-stop is located. The image size is approximately $75 \mathrm{~mm}$. The mount supporting the off-axis telescope optics gives the appearance of being unbalanced. However, large counterweights are used to balance the system and enable very accurate pointing and tracking. A close-up image of M2 is shown in Figure 6. 


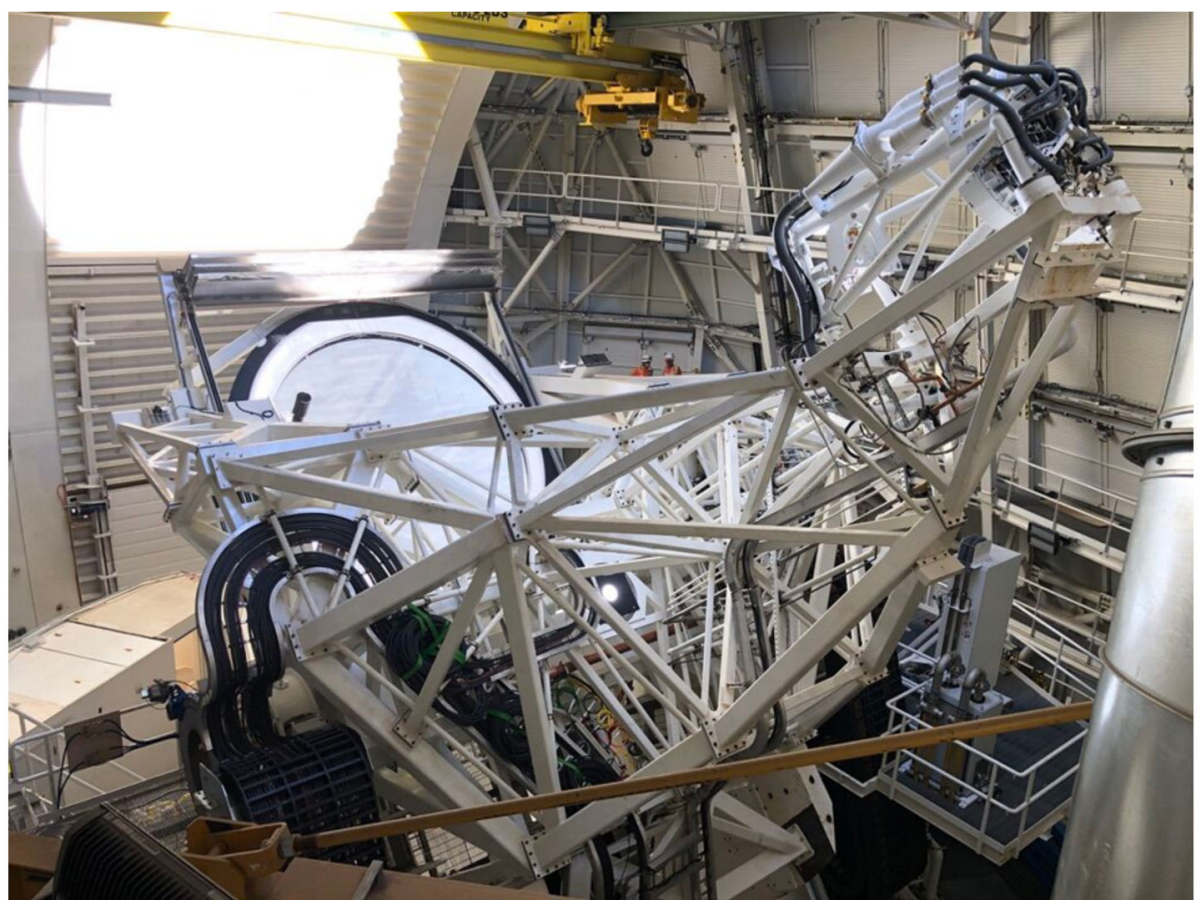

Figure 5 The $4 \mathrm{~m}$ off-axis main telescope at first light. The absorber of the heat-stop (see Section 4.2) was not yet installed at the time of first light. As a consequence the reflected $f / 2$ beam hit the inside of the enclosure.

\subsection{Heat Stop and Lyot Stop}

The heat stop is located at the $\mathrm{f} / 2$ prime focus and is grouped with the Top End Optical Assembly (Canzian et al., 2012). The main function of the heat-stop is to pass the specific 5 arcmin FOV that is of interest onto subsequent optics and the instruments while rejecting approximately $98 \%$ of the incoming $13 \mathrm{~kW}$ of power. The heat stop also serves as an occulting device for off-disk coronal observations. In this case, the solar-disk light is blocked entirely by the heat stop passing the faint coronal features only. The heat flux density at prime focus is $2.5 \mathrm{MWm}^{-2}$. A compact, liquid cooled reflector-absorber combination was designed and built to absorb the $13 \mathrm{~kW}$ load close to prime focus. Unfortunately, the required coolant flow rate through the heat stop was not achievable, likely due to internal contamination of the flow channels. Figure 6 shows an image of the reflecting heat stop, which was installed during first-light commissioning. This temporarily installed device directed the reflected beam onto the inside of the enclosure where it likely caused unwanted heating and seeing effects. An alternate design is in the process of being implemented. The design uses a reflector in combination with an absorbing plate located on the telescope-mount structure a short distance from the reflector. The absorber plate is large enough to intercept the quickly expanding (f/2) beam from the reflector.

A Lyot-stop is placed at the pupil image formed by M2. The Lyot-stop is used to block light diffracted at the edge of the primary aperture stop. The Lyot-stop is motorized and can be deployed for specific coronal applications. The stop is cooled to avoid seeing effects. 


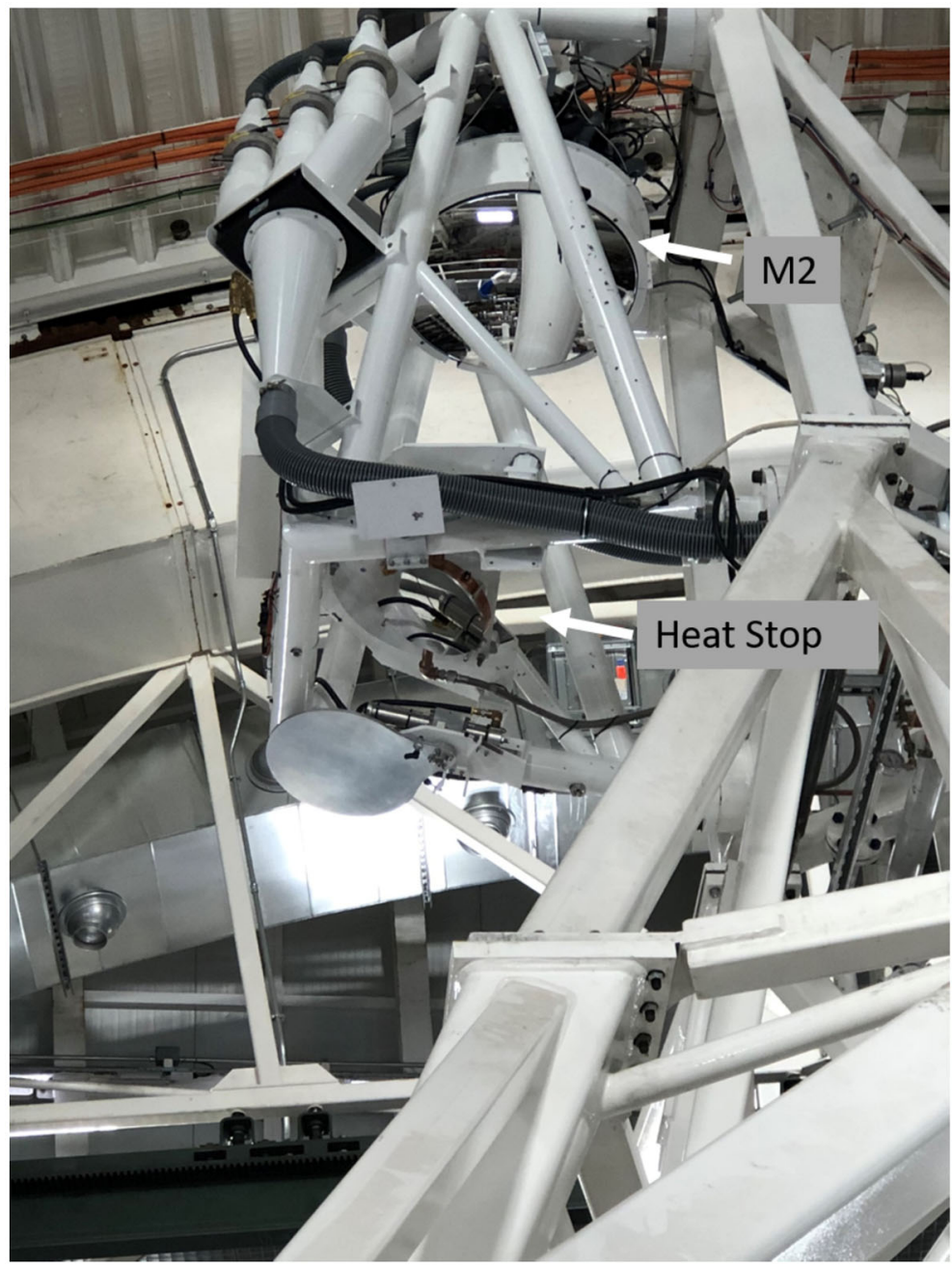

Figure 6 Top-end of the main telescope with heat stop and tip/tilt M2. The reflecting heat stop shown in this image was used during first-light commissioning and will be replaced with a reflector-absorber combination. The M2 has a diameter of $0.635 \mathrm{~m}$.

\subsection{Gregorian Optical Station}

The Gregorian Optical Station (GOS) is located near the f/13 Gregorian focus and houses the polarimetry-calibration optics, calibration targets, pinholes for alignment purposes, and calibration light sources. Figure 7 top shows a detailed mechanical design drawing of the GOS. The actual implementation of the GOS, which in terms of size and complexity compares to an instrument, is displayed at the bottom of Figure 7. GOS elements, including the 2.8 arcmin and 5 arcmin field stops, are mounted on motorized stages and are cooled using liquid cooling or airflow. The GOS moves with the Optical Support Structure. A curved occulting disk for near-limb observations that require minimizing scattered light (e.g. spicules, prominences) is also available at GOS for the two FOVs. The solar limb can be slightly 

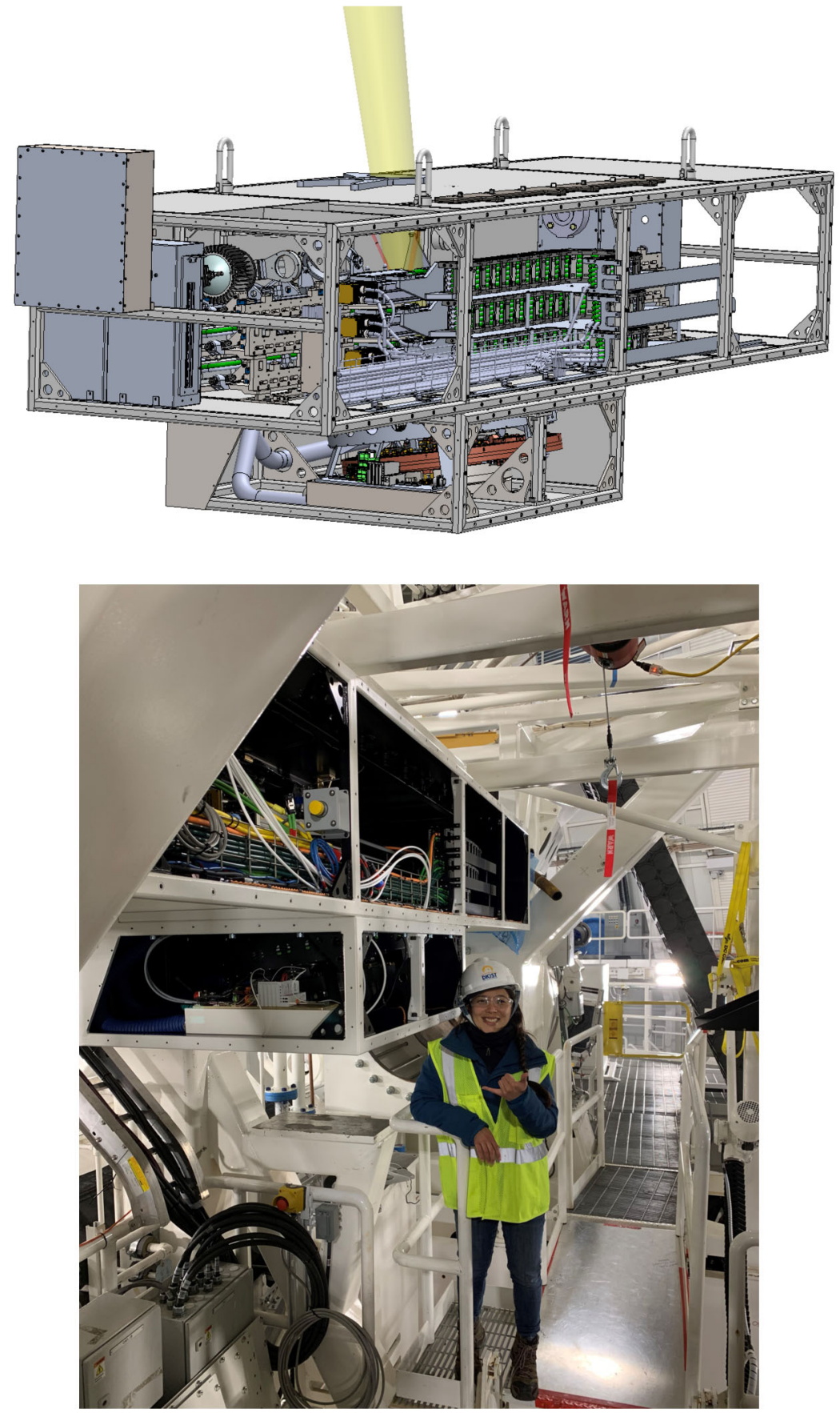

Figure 7 Gregorian Optical Station (GOS). Top: Schematic showing the upper GOS, which holds polarization-calibration optics and the lower GOS with the large rotating stage, which holds field stops, limb occulter, pinholes, and imaging targets (e.g. line grid). The physical dimensions are: for upper GOS $3355 \mathrm{~mm} \mathrm{~L} \times$ $1042 \mathrm{~mm} \mathrm{~W} \times 660 \mathrm{~mm} \mathrm{H}$; for lower GOS $1105 \mathrm{~mm} \mathrm{~L} \times 1042 \mathrm{~mm} \mathrm{~W} \times 406.4 \mathrm{~mm} \mathrm{H}$. 


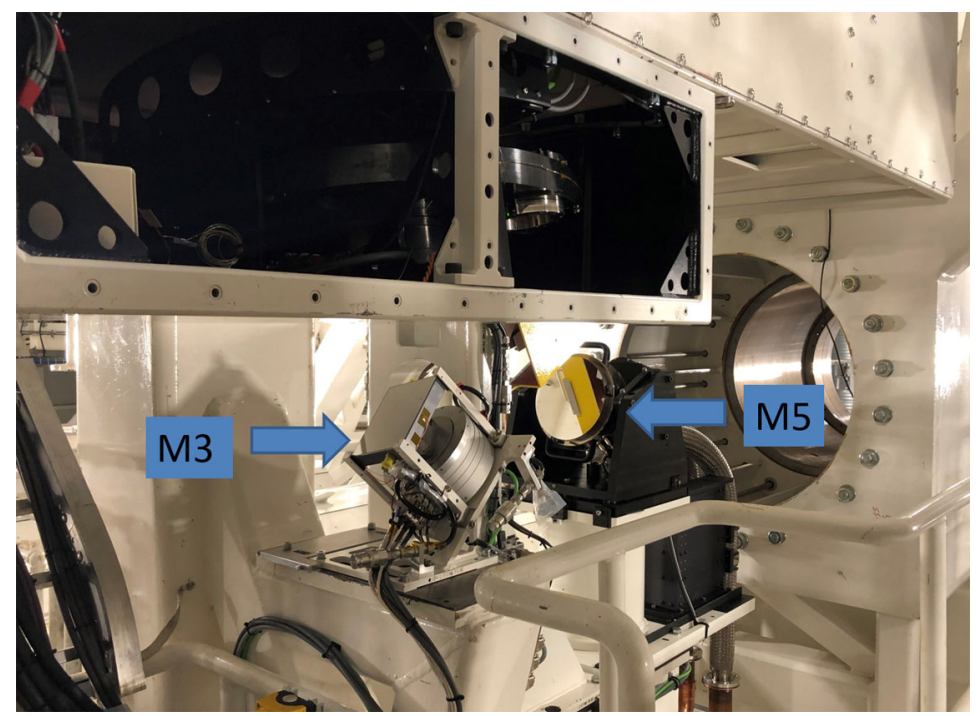

Figure 8 Image of M3, M5, M6 combination, which are part of the transfer optics. M5 has a diameter of $275 \mathrm{~mm}$ and serves as the fast tip-tilt corrector for the AO system. The round SiC mirror (M5) is actively cooled from the back with air jets. The square-shaped M3 is liquid cooled and is used for active pupil alignment. M6 is located just above M5 and is hidden from view. The lower GOS, which contains a motorized wheel that holds field stops and calibration targets, is also displayed (see Section 4.3).

over- or under-occulted and stabilized using a limb-tracking sensor and M2 as the tip-tilt corrector.

\subsection{Transfer Optics}

M3 and M4 combine to place the beam over the elevation axis. M4 is another concave ellipsoid and produces an f/60 beam with an image located at a position about $4 \mathrm{~m}$ above the coudé lab floor. There is currently no instrument access to this image plane but it is conceivable that future upgrades may make use of this focal position. For example, a motorized fiber-feed could be implemented here. The flat mirror M3 and the powered M4 move with the telescope as it points and tracks. The transfer optics also includes flat mirrors M5 and M6 (see Figure 8), which combine to align the beam over the azimuth axis of the telescope mount and the coudé rotator and direct the light beam towards the coudé instrument room. A single mirror at 45 degree inclination would have been sufficient to achieve this function. However, two mirrors each at 22.5 degree inclination were implemented to limit instrumental polarization. In addition, M5 and M6 each provide different functionality. M5 is at a pupil image and provides the fast tip-tilt compensation for the AO system. The system was manufactured by Physik Instrumente. The mirror itself is made from light-weight SiC. Both M5 and M6 have a diameter of $275 \mathrm{~mm}$. As described in Sections 4.8 and 5 the optical design enables active optics and active alignment of the telescope light feed. M6 is located near the same pupil plane and allows (slow) fine-adjustment of the bore-sight. M3 is located near an image plane and is used to keep the pupil image aligned with respect to the actuator grid of the DM. 


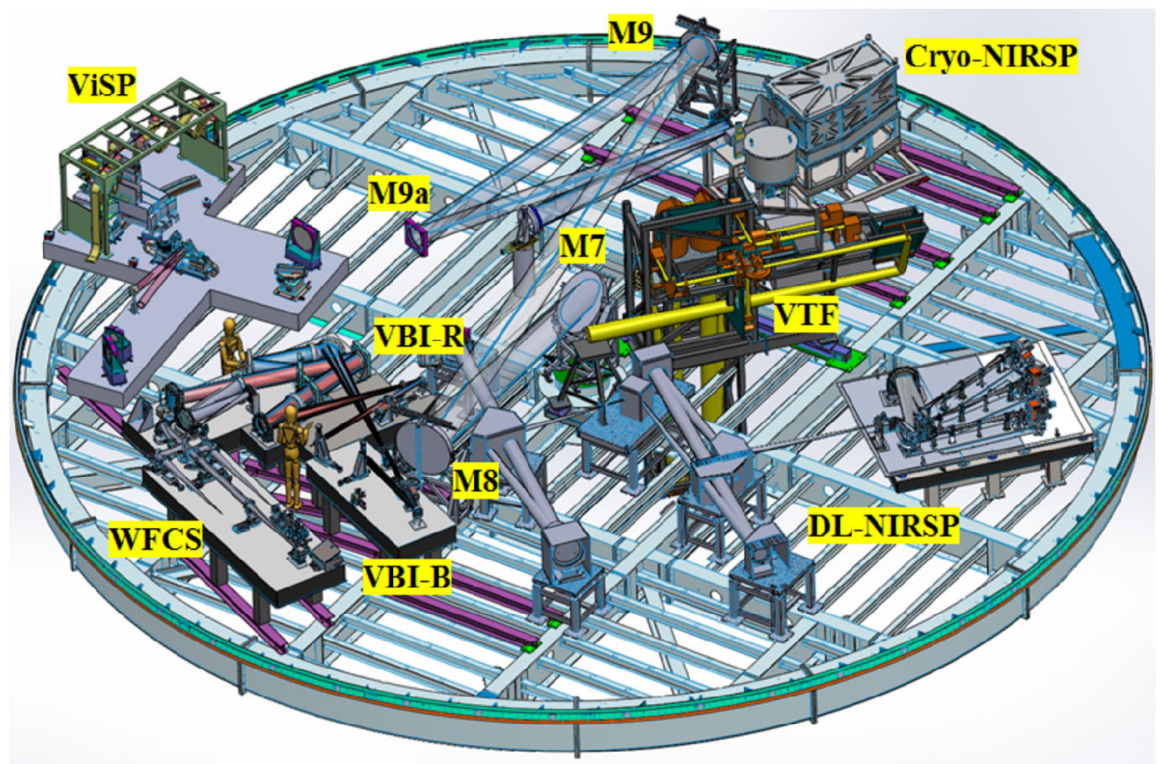

Figure 9 Schematic of coudé rotator with instrument layout and beam distribution. The diameter of the rotator is $16.5 \mathrm{~m}$.

\subsection{Coudé Optics}

The coudé optics includes M7, M8, M9, and M10, which combine to deliver a horizontal, collimated beam and enable the complex, multi-wavelength-band light distribution to the instruments. M7 is a large flat mirror that redirects the light beam parallel to the coude rotator at the correct height for the instruments. M8 is an off-axis parabola that collimates the beam and images the 4 m entrance aperture at M1 onto the DM (M10), which is described in Section 5. M9 is a fold mirror that provides a small amount of coma correction. Figure 9 shows the schematic layout of the instruments in the coudé lab and the complex light-distribution system. The configuration shown with this schematic deploys the mirror M9a to reflect all light to the CRYO-NIRSP in order to, e.g., perform the inherently photon-starved coronal observations at infrared wavelengths. Other instrument configurations require M9a to be shifted out of the light beam. In this case, the light is directed to the DM, which is located on the wavefront-correction (WFC) bench and distributes the light to all other instruments. Figure 10 shows the actual implementation of the transfer optics and instruments, including the $\mathrm{AO}$, in the rotating coudé lab. We note that the size of the optics and the size of the instruments is driven by the $4 \mathrm{~m}$ primary mirror aperture.

\subsection{Instrument Light Distribution}

The configurable facility instrument distribution system (FIDO) enables multiple instrument configurations (Elmore et al., 2014; Harrington, Sueoka, and White, 2019). Multi-instrument experiments are required by many science use-cases, for example, the sampling of the magnetic field at multiple heights in the solar atmosphere. FIDO consists of a number of dichroic beamsplitters, windows, and mirrors. Dichroic beam splitters are used to distribute light to instruments in their specific design spectral ranges with high efficiency. Mirrors can be used 


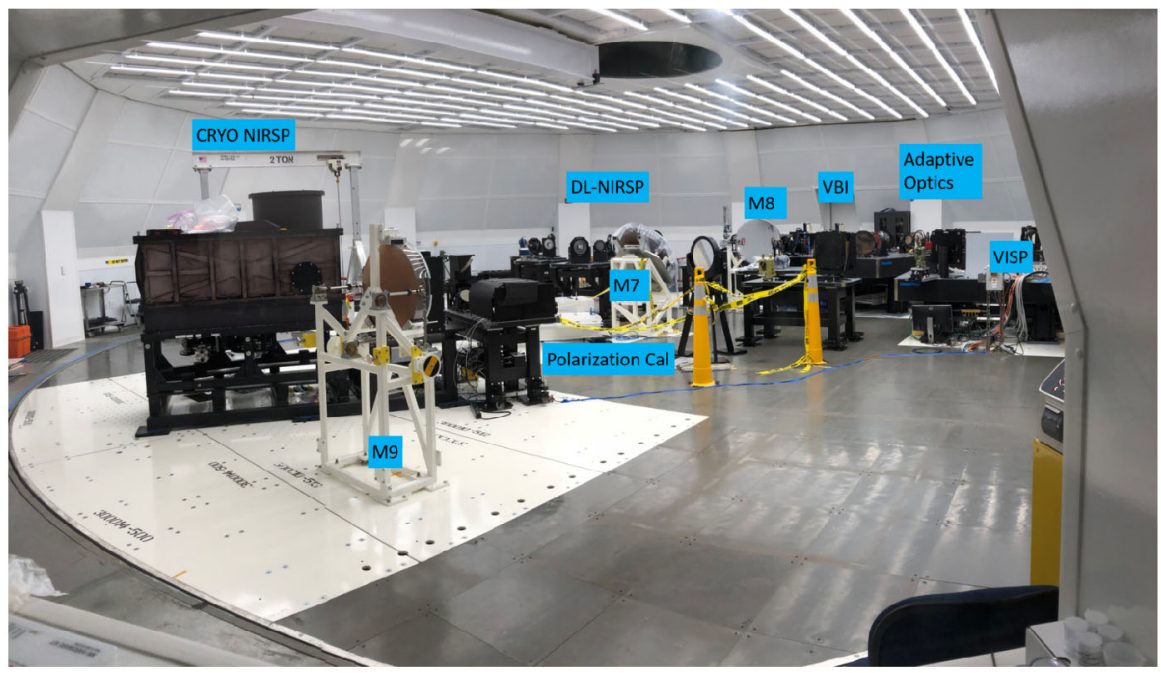

Figure 10 Coudé rotator with instrument beam distribution optics and instruments. The picture was taken during the instrument integration process. The VTF will be added in early 2021. The diameter of the rotator is $16.5 \mathrm{~m}$.

as well, for example, to direct all light to a particular instrument, which for some use-cases is advantageous. A $4 \%$ reflection off a glass window is in some cases sufficient to provide context imaging. For most configurations, FIDO diverts short and passes long wavelengths with each successive beam-splitter encounter. Due to their versatility and flexibility, the spectral ranges of the DKIST instruments often overlap; the use of a particular type of instrument within a given wavelength band often depends critically on the scientific goal of the experiment. Software tools have been developed that enable the users to explore options to combine instruments and verify the feasibility of desired configurations. Additional dichroic and other types of beam splitters are likely to be added during operations. Changing from one instrument configuration to another involves changing beam splitters and some realignment activities. This is a manual process that may require up to one day to complete. Hence, DKIST operations plan to minimize the changeovers within proposal cycles. An exception is the light distribution to the CRYO-NIRSP, which is fed by a motorized mirror (M9a; see Figure 9) inserted at the location M9. Operational concepts dictate a daily and fast (tens of minutes) switch and re-configuration from on-disk, AO aided observations to off-limb coronal observations with CRYO-NIRSP. The CRYO-NIRSP receives all of the light and at this point cannot operate simultaneously with any of the other DKIST instrumentation or with the AO system. However, a beam-splitter option will be implemented during early operations to include the CRYO-NIRSP in some selected multi-instrument experiments. The wavefront correction (WFC) beam splitter is installed permanently and diverts $4 \%$ of the light to the active-optics and AO wavefront sensors and the WFC context viewer.

\subsection{Thermal Control of Optics}

Minimizing locally induced seeing effects along the optical path is crucial to meeting the challenging imaging error budgets, which flow from the top-level imaging performance requirements. Without active cooling, mirror surfaces can heat to several degrees above ambient air temperature, depending on many factors including the coating reflectivity, air flow 
(natural or forced) across the mirror surface, material properties (thermal conductivity and thickness), and mounting configurations. The amount of heat absorbed often critically depends on the coating chosen for the optical surface. Most of the DKIST mirrors are coated with high-reflectivity silver coatings. In order to avoid mirror seeing, most optical surfaces are actively controlled to local ambient temperatures during operations to avoid the buildup of convection and associated image distortion. Other mitigation strategies include air knives that provide a laminar air flow across a mirror surface or optical element in order to obtain forced-convection, which greatly reduces image distortion compared to the naturalconvection regime (Dalrymple, Oschmann, and Hubbard, 2004). Of particular importance is temperature control of the primary and mirrors close to pupil planes. The M1 is coated with aluminum and absorbs in excess of $120 \mathrm{~W} \mathrm{~m}^{-2}$. The rather small M1 thickness of $75 \mathrm{~mm}$ was mandated by thermal-control considerations. In order to keep the surface temperature of $\mathrm{M} 1$ between 0 and $-2^{\circ} \mathrm{C}$ of ambient, the front surface of $\mathrm{M} 1$ is controlled by air-cooling the mirror from the back side with 555 air jets. Thinner substrates dramatically reduce the thermal time lag and thus simplify the closed-loop thermal control. Modeling and tests have confirmed the ability of the M1 cooling system to control the M1 mirror surface to specifications (Hansen, Price, and Hubbard, 2006; Hansen, Bulau, and Phelps, 2008). Air-jet cooling from the back side is also used to cool smaller, fast tip-tilt mirrors. In order to achieve the required frequency response, these mirrors have a tight mass budget, i.e. liquid cooling is not an option. This cooling option is implemented for M2 and M5, which absorb up to $60 \mathrm{~W} \mathrm{~m}^{-2}$ and $390 \mathrm{~W} \mathrm{~m}^{-2}$ (5 arcmin FOV), respectively (Cho, DeVries, and Hansen, 2007). M3 (2 $\mathrm{kW} \mathrm{m}^{-2}$ absorbed) and M6 (350 $\mathrm{W} \mathrm{m}^{-2}$ absorbed) are made from silicon and are both liquid cooled. The coudé optical elements M7, M8, and M9 absorb relatively small amounts of energy and are not close to pupil planes. For these optics, providing air flow across the surface either by the laminar flow present in the entire coude lab or by air knives installed at the mirror surface suffices to mitigate any seeing effects. The silver-coated deformable mirror (M10) is located at a pupil plane and absorbs $90 \mathrm{~W} \mathrm{~m}^{-2}$. The DM's face sheet is made from highly conductive silicon and is attached to a silicon block that serves as a heat sink, which is thermo-electrically cooled. FIDO optical elements including dichroic beam splitters are exposed to the laminar downward air flow of the instrument lab and are not actively cooled. A complex Facility Thermal Control System provides master control to the optical elements (White and Phelps, 2016).

\subsection{Alignment Strategies}

Aligning an off-axis optical system is significantly more challenging than aligning symmetric optics. The alignment process is described by Sekulic et al. (2016) and was informed by detailed analysis, modeling, and simulations. The use of modern metrology equipment such as laser trackers, coordinate measuring machines, interferometers, and theodolites greatly alleviates alignment challenges. A wavefront sensor using point sources (stars) was deployed at the various focal stations, namely prime focus, Gregorian focus, and coudé focus during the initial system alignment process. In combination with other metrology tools, the wavefront-sensor facilitates successive alignment of optical elements and groups. For example, M1 is positioned using a wavefront sensor at prime focus. M2 is aligned with M1 using a wavefront sensor mounted at the Gregorian focus. The results of the alignment procedures are summarized by Sekulic et al. (2020). In addition to the initial, passive alignment, active-alignment strategies using wavefront sensors and look-up-tables (LUT) are deployed during operations as described in Section 5. 


\section{Wavefront Correction System}

The wavefront-correction system is a key component of DKIST (Rimmele et al., 2006; Richards et al., 2010; Johnson et al., 2016) that enables both seeing-limited and diffractionlimited observations. This subsystem includes:

i) A solar $\mathrm{AO}$ system that corrects atmospheric seeing and residual optical aberrations at 2 $\mathrm{kHz}$ rates. The main components of AO are the 1600-actuator Deformable Mirror (DM, manufactured by Northrop Grumman AOA Xinetics (Cavaco and Wirth, 2014)), and a fast tip-tilt mirror (M5, Physik Instrumente). The wave-front sensor is a correlating Shack-Hartmann sensor (Rimmele and Marino, 2011) with 1457 sub-apertures.

ii) An active-optics $(\mathrm{aO})$ system that corrects slowly changing aberrations that may arise primarily from gravitational and thermal deformations of the telescope structure. The $\mathrm{aO}$ system ensures that the $\mathrm{M} 1$ surface figure remains within allowed tolerances during operations. The hexapod-mounted M2 is also used as a corrector primarily for focus (z-motion) and astigmatism (decenter).

iii) Active alignment. Wavefront sensors and LUT information are used to keep pupil images and boresight in alignment. M3 and M6 are used to correct small drifts that might occur, for example, while the coudé rotator turns around its axis.

As discussed in Section 2.1, simulations of observables were performed early on to guide the requirements development. Simulated observations based on MHD simulations (Stein and Nordlund, 2006), for example, of the mixed polarity fields generated by surface dynamo action strongly indicated the need for an AO system that delivers high-Strehl imaging in order to recover sufficient quantitative information. A Strehl requirement of $\mathrm{S}>0.3$ for $\mathrm{r}_{0}(500 \mathrm{~nm})>7 \mathrm{~cm}$ (close to median seeing) and a Strehl requirement of $\mathrm{S}>$ 0.6 for $\mathrm{r}_{0}(630 \mathrm{~nm})>20 \mathrm{~cm}$ were derived in this way (Rimmele and the ATST Science Working Group, 2005). The high Strehl requirement defines the expected imaging performance for excellent seeing conditions, during which high-priority science objectives will be achieved. Imaging error budgets and many system and subsystem requirements flow from these two top-level specifications. It should be noted that the performance requirement for median-seeing conditions are more difficult to achieve compared to the excellent seeing requirements due to, e.g., larger fitting and bandwidth errors. The fitting error is due to the limited ability of the DM to reproduce, and hence correct, high-order wavefront errors. The bandwidth error is a result of the finite $(2 \mathrm{kHz})$ update rate of the AO. During the systems-engineering process and also driven by science-productivity considerations, the number of actuators and sub-apertures was increased to 1600 and 1457 respectively, compared to earlier designs (Rimmele et al., 2006). The AO system is capable of correcting up to approximately 1400 Karhunen-Loève (KL) modes. The high order of correction achieves the required Strehl ratio but also enables the system to more gracefully handle rapid and sometimes significant dips in the seeing parameter $r_{0}$ that are common for daytime seeing.

The $\mathrm{aO}$ system operates on timescales of tens of seconds to minutes. M1 figure control and alignment functions can be performed with previously established LUTs or using wavefront-sensor outputs from either the $\mathrm{AO}$ or aO, respectively, depending on the operational mode. Both the aO wavefront sensor (Johnson et al., 2012; Johansson et al., 2018) and $\mathrm{AO}$ wavefront sensors can provide time-averaged wavefront measurements. By averaging over the atmospheric seeing, information about slowly varying aberrations due to optical misalignment and/or M1 figure deformations is provided to the wavefront control system. The system architecture and the optical design of the wavefront sensors and context viewer were described in much detail in previous publications (Rimmele et al., 2006, 2010; 

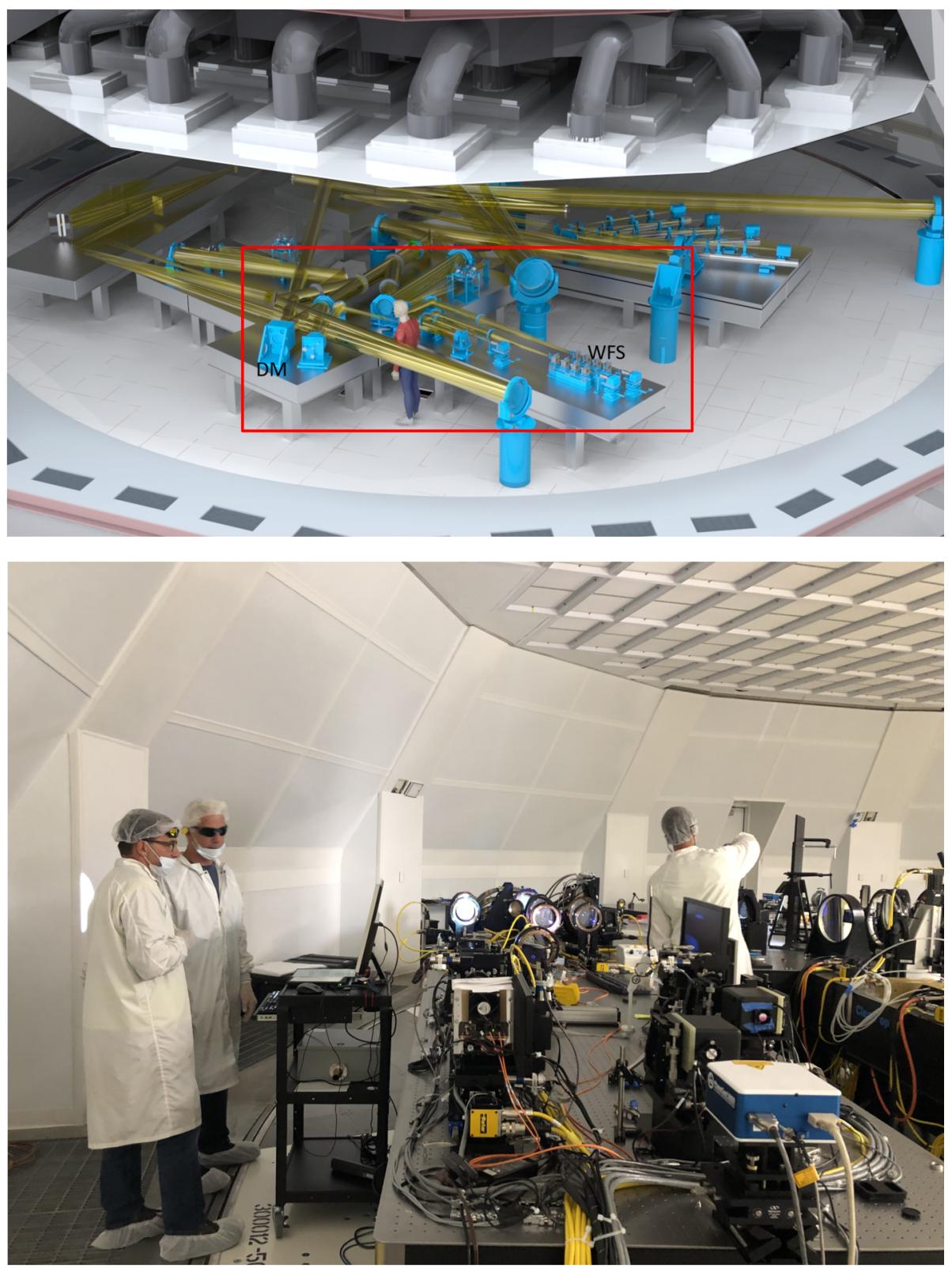

Figure 11 Top: Mechanical design of the wavefront-correction bench that holds the DM, the high- and low-order wavefront sensors, and the context viewer. The elaborate HEPA filtration system that provides the laminar downflow to the coudé lab is also visible. The red box highlights the wavefront-correction bench. Bottom: Wavefront-correction bench during first solar light achieved at the coudé instrument lab. The diameter of the rotator is $16.5 \mathrm{~m}$.

Richards et al., 2010; Johnson et al., 2014, 2016). Integration and system testing are summarized by Johnson et al. (2018, 2020). The Field-Programmable-Gate-Array (FPGA) based 
real-time control of the $\mathrm{AO}$ and its integration into the overall active- and adaptive-wavefront control concept are described by Richards et al. (2010).

Figure 11 top shows a design schematic of the wavefront-correction system located in the coudé lab. A life-size person has been inserted into the graphic to give a size scale. The 1600 actuator DM receives drive signals from the FPGA-based reconstructor at a rate of $2 \mathrm{kHz}$.

First on-sky data with the wavefront-correction system in operation were obtained during the first-light initiative in December 2019. Figure 11 bottom shows an image of the wavefront-correction system receiving its first solar light. The high- and low-order wavefront sensors and the context viewer, which produced the sunspot image shown in Section 14, are displayed with this image. We note that staff can only enter the coudé instrument lab while wearing proper clean-room equipment. At the time of first solar light, the system was not optimized and not all thermal-control systems were operational. A knife-edge test confirmed presence of local-seeing effects that have yet to be addressed. Nevertheless, the AO system delivered Strehl ratios as estimated from wavefront-sensor telemetry very close to specifications (Johnson et al., 2020), enabling the diffraction-limited first-light imaging presented in Section 14.

We note that, although not part of the construction-project scope, an upgrade from conventional AO to MCAO (Schmidt et al., 2017) was envisioned early on in the project. The optical design can accommodate additional DMs at conjugates of turbulence layers in the upper atmosphere. M7 and M9 are conjugate to $11 \mathrm{~km}$ and $4 \mathrm{~km}$ height in the Earth's atmosphere, respectively, and thus in near-optimal location for a three DM MCAO system. Design and prototyping efforts have been ongoing (Schmidt et al., 2018) and additional DMs that will replace M7 and M9 are currently in their design or manufacturing phase, respectively.

\section{Polarimetry}

DKIST is constrained by its demanding polarimetry requirements. The DKIST SRD summarizes a diversity of observing scenarios. Many observing use-cases call for simultaneous observations of multiple wavelengths spanning ultraviolet to infrared wavelengths. Instruments have a wide range of polarimetric sensitivity and accuracy requirements. The DKIST project has undertaken a large system-level effort to understand the polarization performance and limiting errors covering a very wide range of scenarios.

A common way of summarizing polarization errors in an optical system is to describe how errors in various sub-matrices within the system Mueller matrix impact the accuracy of the reconstructed Stokes vector for the solar beam. We emphasize that the optical system includes the telescope, relay optics, FIDO dichroics, instrument feed optics, and the instruments. Any configuration-dependent changes such as instrument, wavelengths, or optics positions in any of these sub-systems are tracked in the DKIST databases. For instance, the orientation of the measured Stokes vector directly couples to the retardance $3 \times 3$ sub-matrix. The magnitude of the Stokes vector can be impacted by the depolarization row and the polarizance column. The demodulation matrix can influence all of these parameters (see, e.g., Section 7, Harrington and Sueoka, 2018a). Several of the science use-cases call out a continuum polarization stability (accuracy) specification of $0.05 \%$ for the polarizance column. As with any optical system, the main accuracy limitations are instabilities in the system. If the system calibrations are stable and account for the relevant sources of error, accuracy can be achieved for relatively long durations even with instruments changing their optical configurations (e.g. scanning a field by moving a steering mirror). Others require a polarization 
sensitivity of $0.001 \%$ limited by photon statistics and the system throughput. These limits typically apply after substantial temporal averaging, spatial smoothing, spectral binning, or within some limiting field of view to achieve the required sensitivity.

DKIST has expended substantial effort towards understanding and minimizing polarization-error sources. An inter-disciplinary set of new modeling mathematics and algorithms coupled with new laboratory tools for detailed characterization of optical elements was developed. Mueller-matrix spatial-spectral mapping systems covering UV to IR wavelengths were developed and utilized to characterize optics (Harrington et al., 2020a). Retarders were spatially mapped, characterized for elliptical retardance, and this ellipticity is included in the calibration process. Full-sized optics were fabricated and thermally stress-tested to assess temporal stability in both alignment and polishing. New coatings were developed in collaboration with vendors to mitigate interference fringes (see Harrington et al. (2021) and the references therein). The mirror-coating polarization was measured for every coated surface in the system including all instrument optics and facility dichroics (Harrington and Sueoka, 2017; Harrington, Sueoka, and White, 2019). The coatings and their impact on system calibration were modeled to understand how to use the AO system and field-scanning techniques to keep polarization errors below user-defined thresholds. A dual fiber-fed spectrograph system was designed and deployed to calibrate DKIST, including the CRYO-NIRSP feed optics and covering $390 \mathrm{~nm}$ to $1650 \mathrm{~nm}$, which represents the majority of the proposed science use-cases for early operations. Preliminary system model calibrations were successful and $>100$-variable fits are well within tolerance expectations for the properties of DKIST optics (Harrington et al., 2020b). The suite of facility dichroics that feed the AO-assisted instruments have open-source coating designs that have been characterized for their polarization performance in both reflection and transmission and also for spatial uniformity of coatingpolarization properties (Harrington, Sueoka, and White, 2019).

These efforts have given DKIST a detailed system-level knowledge of the optical performance. Thorough testing of instrument-level alignment stability and camera-sensor performance provide an understanding of errors within each facility instrument. Additionally, system-level polarization calibration activities are planned for early operations to test the accuracy using line-correlation techniques (Elmore et al., 2010; Derks, Beck, and Martínez Pillet, 2018), daytime sky observations (Harrington et al., 2015; Harrington, Kuhn, and Nevin, 2015; Harrington, Kuhn, and Ariste, 2017; Harrington, Kuhn, and Hall, 2011), unpolarized and polarized star observations (Whittet et al., 1992; Hough et al., 2006; Fossati et al., 2007; Bailey et al., 2008; Bailey, Lucas, and Hough, 2010; Harrington, Kuhn, and Nevin, 2015), and comparison with some of the most accurate continuum-polarization measurements from IRSOL (Bianda, Ramelli, and Gisler, 2009).

\section{Major Mechanical Systems}

The telescope mount and the coudé rotator combine to enable accurate and precise pointing and tracking at both the Nasmyth and coudé instrument stations. The enclosure protects the telescope from the outside environment, and it is designed to provide optimum observing conditions inside the enclosure. Pointing and tracking requirements are also placed on the enclosure. The fast $\mathrm{f} / 2$ design was chosen to allow for a relatively compact telescope mount structure; the Gregorian optical design and, to some extent, the off-axis design drive the size of the telescope facility - in particular the enclosure. Even though DKIST's aperture is $4 \mathrm{~m}$, the facility compares in size to $8-10 \mathrm{~m}$ night-time telescopes and in many ways exceeds them in complexity. 


\subsection{Telescope Mount and Coudé Rotator}

The alt-az telescope mount supports the M1 - M6 mirror assemblies and provides for slewing, pointing, and tracking throughout altitude and azimuth rotations (Warner et al., 2006). The SRD requirements call for absolute (blind) pointing to be accurate to $<5$ arcsec. An offset pointing accuracy of 0.5 arcsec or better was specified. Long exposures may be required for certain coronal use-cases where active guiding of very faint coronal features is currently not possible. A tracking stability of better than 0.5 arcsec over a period of one hour is the performance requirement. The required off-pointing range is 1.5 solar radii in all directions. The off-pointing is essentially limited by the physical dimension of the heat stop and could be increased in the future. Additional specifications included tracking accuracy as a function of speed, slew rate, range of motion, and allowable jitter. In particular, jitter specifications are challenging, driven by imaging error budgets (Jeffers et al., 2016). Another challenge in designing the telescope mount was the non-symmetric nature of the optical layout and resulting imbalanced and cantilevered loads. Compounding these large non-symmetries was the requirement that all optic positions not only be held accurate with respect to each other, but also with respect to the so-called "cross-point" where the azimuth and altitude axes coincide. Light must be delivered along these axes and, ultimately, be co-aligned with the rotation axis of the coudé lab, to avoid beam wobble. For the azimuth axes mechanisms for both telescope mount and coudé rotator, bearing and drive technologies were used similar to what has been developed for the machine-tool industry (Kärcher et al., 2012).

As an example of a major mechanical system, Figure 12 shows the coudé-rotator platform. A design schematic of the platform with all instrumentation installed is shown at the top of Figure 12. Additional space for potential future instrument upgrades is located underneath the floor and in the center of the rotator. The VTF is currently using some of this space. Also seen are cooled electronic racks for instruments and wavefront-correction systems. The coudé rotator platform is 16.5 meters in diameter and weighs approximately 115 tons. Given its size, mass, and the criticality of maintaining a precise optical path to the instrument detectors, the coudé rotator has exacting requirements on its motion and deviations from that motion (deflection and jitter) as it tracks a target. For example, a lateral bearing run-out of approximately 50 micron has been achieved for this massive structure. The telescope mount and coudé rotator were designed by MT Mechatronics, and fabricated by Ingersoll Machine Tools. In order to minimize integration challenges on the summit, the telescope mount and the coudé rotator, both massive systems, were assembled and tested in the factory. Detailed factory testing demonstrated that these systems met all requirements before they were disassembled, packaged, and shipped to the summit for installation and further testing and site acceptance (Jeffers et al., 2016). Figure 12 bottom shows the fully assembled coudé-rotator platform during factory acceptance testing at Ingersoll Machine Tools.

A first light pointing model was established from observations of 100 night-time objects. The model achieves a pointing accuracy of 2.65 arcsec measured in the coudé lab. The data indicate residual systematic pointing errors and an improvement by a factor of two may yet be possible. A solar pointing model has yet to be established but is expected to be of similar accuracy.

\subsection{Enclosure}

The enclosure consists of the structure and mechanisms required to track and protect the telescope, providing a clear optical path to the Sun but preventing illumination and subsequent heating of any structures outside the optical path, as well as supporting the thermal 
T.R. Rimmele et al.
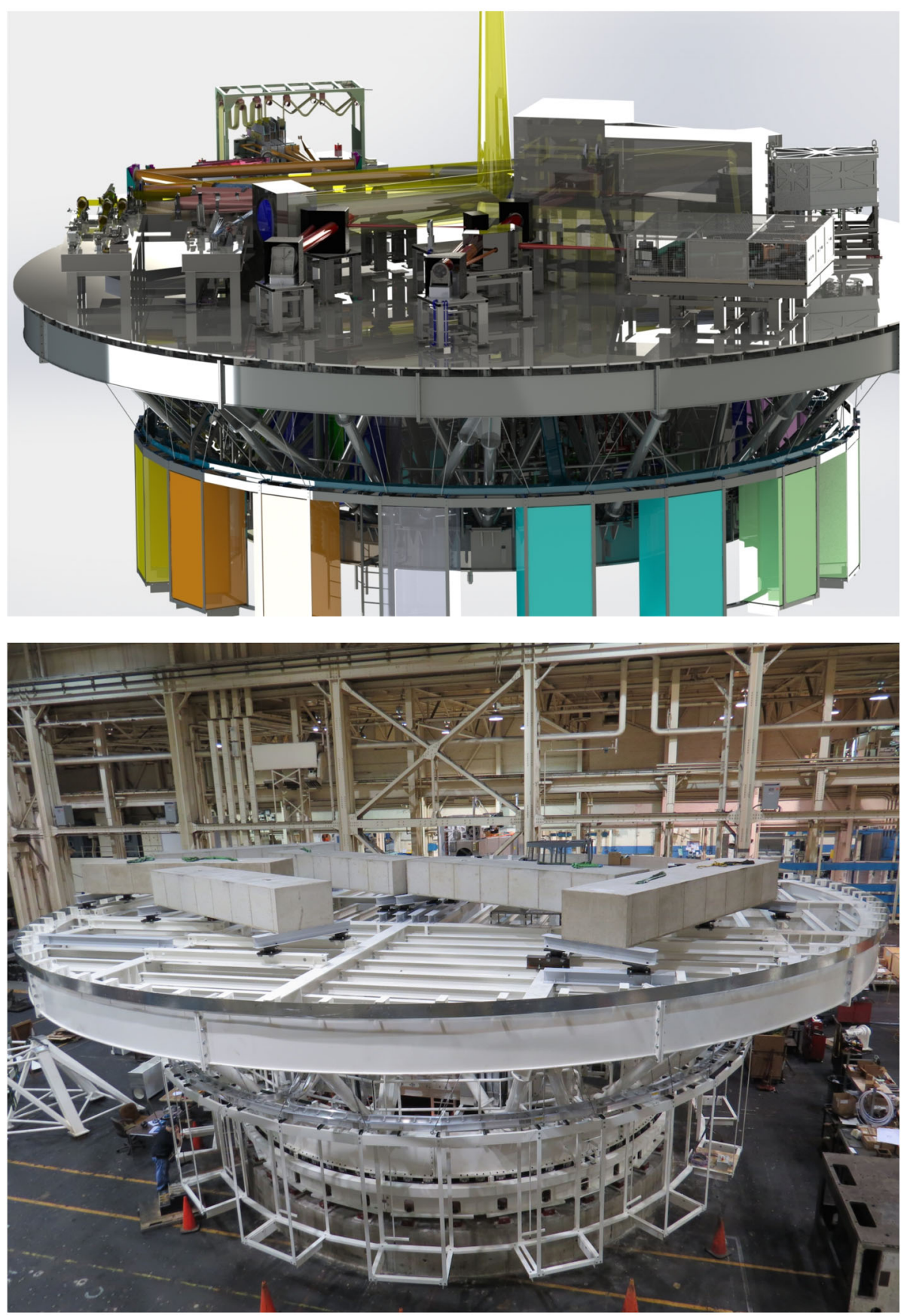

Figure 12 Top: Mechanical design rendering of the $16.5 \mathrm{~m}$ diameter coudé rotator with instruments. Bottom: coudé rotator at the Ingersoll factory where the 115 metric ton structure was fully assembled and tested. Dummy masses were used to emulate the instrument loading. 
conditioning of the facility. The decision for a co-rotating, ventilated enclosure (Murga et al., 2012) was made early on. This solution provides protection from the occasionally harsh environmental conditions including severe ice storms. The enclosure was also designed to avoid enclosure and dome seeing (Dalrymple, Oschmann, and Hubbard, 2004). Sufficient ventilation of the inside of the enclosure is paramount. A large number of motorized and computer-controlled vent gates provide the ability to adjust and optimize air flow through the enclosure and across the M1. For example, in high-wind conditions excessive jitter and deformations of the $75 \mathrm{~mm}$ thick M1 due to wind buffeting can be mitigated by throttling wind flow through the enclosure (Hubbard and Oschmann, 2004). Active ventilation systems were included for use during low-wind conditions. The enclosure also protects telescope structure and the large floor area around the telescope mount from direct sunlight and resulting seeing effects. To avoid dome seeing, critical sections of the enclosure exterior are actively cooled (Phelps et al., 2014).

The enclosure has a height of $22 \mathrm{~m}$ and a diameter of $26.6 \mathrm{~m}$. Its weight of approximately 750 metric tons is supported by the lower enclosure (Phelps and Warner, 2008). The overall facility height is $41.4 \mathrm{~m}$ (Murga et al., 2014). The tracking of the enclosure and the solar aperture must be done with great precision to keep the solar beam centered on the aperture stop of the M1. Similar to the Telescope Mount and coudé rotator and, for the same reasons, the enclosure was pre-assembled at the IDOM factory to verify specifications and establish and document the assembly process before transporting it to the Haleakalā summit (Murga et al., 2014). The site-integration effort on the summit proceeded relatively smoothly.

\section{Facility Thermal Systems}

Minimization of seeing effects was a major design consideration. The infrastructure implemented to provide the thermal control and thus internal-seeing mitigation strategies is substantial and complex. Thermal design concepts and analyses have been discussed in previous sections and the publications listed there (Phelps et al., 2012). In addition to the thermal control of optical surfaces, structural elements are controlled to ambient temperatures. For example, sections of the dome skin are actively cooled (Phelps et al., 2014; Gorman et al., 2016). The coudé lab is temperature stabilized to $20 \pm 0.5^{\circ} \mathrm{C}$, which ensures stability of instruments and their calibrations (Phelps et al., 2010). An interesting detail is the air knife that separates the environmentally controlled coudé lab from the enclosure environment. While the coudé lab is maintained to $0.5{ }^{\circ} \mathrm{C}$ at $20{ }^{\circ} \mathrm{C}$, the inside of the enclosure experiences large swings as the outside temperature changes during the day and during the year. To avoid absorption of IR wavelengths, a glass window cannot be used to separate the two environments. The air knife serves that function instead (McQuillen et al., 2016; McQuillen and Phelps, 2018). The air knife is installed along the optical path between M6 and M7 just above the f/60 focal plane shown in Figure 3 and provides a strong, laminar cross flow.

During day-time operations, cooling capacity is provided by the ice storage tanks seen in Figure 13 bottom. Ice is made during the night when electricity is less expensive. Approximately 12 kilometers of pipes distribute coolant throughout the facility. Figure 13 top shows the inside of the utility building and gives a sense of the complexity of the thermal-control and distribution system. Three large air-handling units are part of the thermal-control system. A sophisticated control system provides master control to the distributed elements in order to safely and economically monitor and operate the thermal equipment and control site power demand (White and Phelps, 2016). 

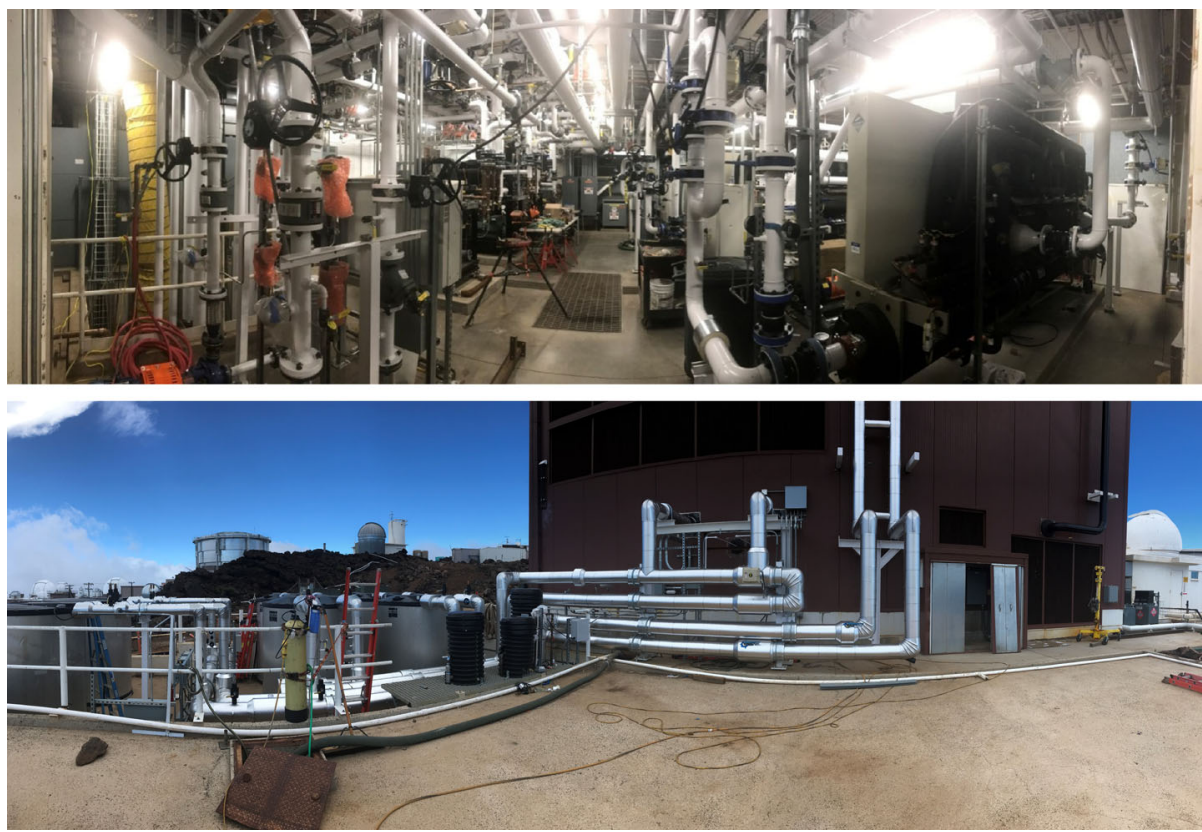

Figure 13 Inside of the utility building showing an example of the complex thermal systems infrastructure (top). Ice storage tanks next to the utility building (bottom). Also visible is the large dome structure of the AMOS telescope where the coating facility is located.

\section{Support Structures and Systems}

The massive telescope pier that supports the telescope and coudé rotator is described and analyzed by Jeffers et al. (2012a). The telescope pier is isolated from the lower enclosure to avoid wind- or motion-induced vibrations from the enclosure. A control room, from which telescope and instruments are operated and monitored, is located in the Support and Operations building. A "mirror" control room is located at the DKIST Science Support Center (DSSC) in Pukalani, Maui, Hawai'i (see Figure 14). In principle, telescope and instruments could be controlled remotely from the DSSC. However, for early operations this capability will not be enabled. The expectation is that after several years of operations experience, remote operations will be established gradually. Initially, the remote control room will be utilized by the Resident Scientists to guide and monitor service-mode operations (see Section 12).

\section{Software Systems}

Top-level requirements for software systems flow from the Operational Concepts Document. The Observatory Control System (OCS) is the main user interface from which operators control the telescope and instruments (Wampler and Goodrich, 2004). The OCS performs the experiments and observation requested by the observer. It controls and coordinates the operations of the telescope and instrument through the user interfaces and experiment scripts. The Telescope Control System (Goodrich and Wampler, 2004) built by Observatory Sciences controls the configuration of the telescope and its subsystems during 


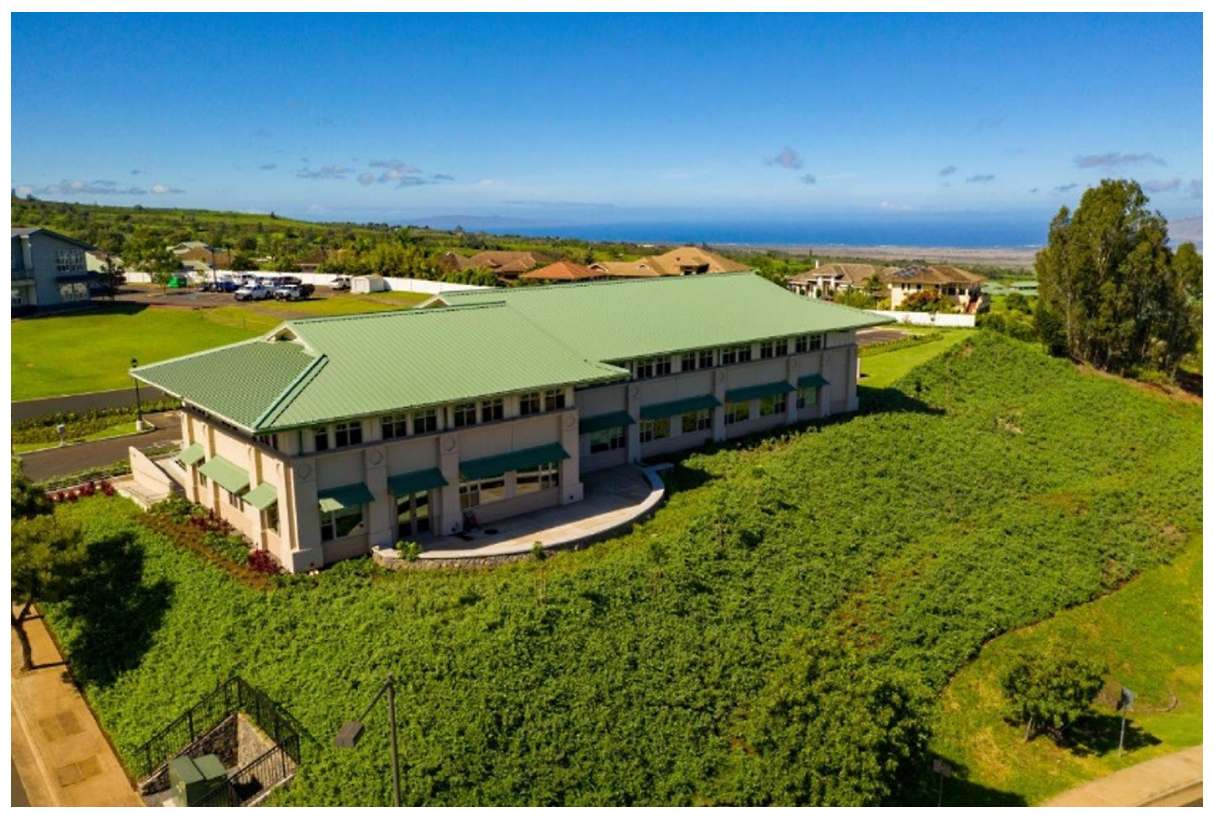

Figure 14 The DKIST Science Support Center (DSSC) in Pukalani, Maui, Hawai'i. This base facility provides office and lab space and includes a remote operations room from which observations can be monitored and guided by science staff.

science operations, including the pointing model for the telescope mount and optical elements, a solar ephemeris with several coordinate-tracking and rotation systems, a thermalmanagement system, and a wavefront-management system. The Instrument Control System (ICS) is the principal system responsible for the operation of the instruments, including the instrument cameras (Johansson and Goodrich, 2012). The ICS supports instrument operations in a synchronized environment. The ICS manages the observing modes of the instruments and ensures their synchronization with each other, the polarizers, and the current observing mode of the facility. The ICS operates under the control of the Observatory Control System (OCS), managing all aspects of instrument operation during an experiment. The Camera System Software provides a common interface to a variety of visible and infrared observatory camera systems and enables their integration with instruments and the datahandling system. The Common Services Framework (CSF) provides the infrastructure for the software control systems (Hubbard, Goodrich, and Wampler, 2010). Services such as connections, events, logging, properties, databases, data headers, and others are integral to the CSF infrastructure.

\section{Instrumentation}

The initial set of facility instrumentation includes the Visible Broad-band Imager (VBI: Wöger et al., 2021), the Visible Spectro-Polarimeter (ViSP: de Wijn et al., 2021), the Diffraction-Limited Near-Infrared Spectro-Polarimeter (DL-NIRSP: Jaeggli et al., 2021), the Cryogenic Near-Infrared Spectro-Polarimeter), (CRYO-NIRSP: Fehlmann et al., 2021), and the Visible Tunable Filter (VTF: von der Lühe et al., 2021). All instruments, except for the CRYO-NIRSP, are provided with an AO-corrected beam. 
The extensive instrument capabilities are briefly summarized in Table 2 and the following sections.

\subsection{Visual Broadband Imager}

The VBI is designed to provide diffraction-limited images and movies at a number of wavelengths that image the photosphere and the chromosphere. A filter that enables highresolution imaging of coronal structures is also included. The VBI and its science mission, and capabilities are described in several previous publications (Wöger et al., 2012; Wöger, 2014). The instrument is divided into a red and a blue channel. In combination, the two channels cover the wavelength range $390 \mathrm{~nm}$ to $860 \mathrm{~nm}$. The CMOS cameras provide a $4 \mathrm{k} \times 4 \mathrm{k}$ image stream at a frame rate of up to $30 \mathrm{~Hz}$. The VBI achieves the full resolution potential of the $4 \mathrm{~m}$ DKIST by using $\mathrm{AO}$ and quasi-real-time speckle image reconstruction at a three-second cadence (Wöger et al., 2010). A summary, the implementation of VBI into the DKIST coudé lab, and first results are described by Wöger et al. (2021).

\subsection{Visible Spectro-Polarimeter}

The ViSP provides precision measurements of the full state of polarization (i.e. all four Stokes parameters $I, Q, U$, and $V$ ) simultaneously at diverse wavelengths in the visible spectrum, while fully resolving the spectral profiles of spectrum lines originating in the solar atmosphere. Such measurements provide quantitative diagnostics of the magnetic-field vector as a function of height in the solar atmosphere, along with the associated variation of the thermodynamic properties. Wavelength diversity is a key element of the instrument allowing the ViSP to simultaneously perform spectro-polarimetric maps in up to three arbitrarily chosen and widely separated lines in the visible and near-infrared spectral range (380 nm$900 \mathrm{~nm}$ ). The High Altitude Observatory has been leading the design and implementation of the ViSP (Nelson et al., 2010; de Wijn et al., 2021).

\subsection{Visible Tunable Filter}

The VTF enables rapid imaging spectroscopy and polarimetry. The dual-etalon Fabry-Pérot filter covers the wavelength range from $515 \mathrm{~nm}$ to $860 \mathrm{~nm}$. The spectral resolution of the Fabry-Pérot (FP) filter system is $6 \mathrm{pm}$ at $600 \mathrm{~nm}$. The FOV is limited by the physical size of the FP to 1 arcmin. The $4 \mathrm{k} \times 4 \mathrm{k}$ pixel detectors enables Nyquist sampling at visible wavelengths providing a spatial resolution of $0.028 \mathrm{arcsec}$ on the sky (20 km on the Sun). A full Stokes-vector measurement in two spatial dimensions and with a polarimetric sensitivity of $5 \times 10^{-3}$ can be recorded within 13 seconds. The VTF uses three detectors: two for the polarimetric measurements, and one for broadband filtergrams. The science goals and main scientific observables, instrument requirements, design, and performance modeling were described by Kentischer et al. (2012) and Schmidt et al. (2014, 2016). The VTF design and construction is being led by the Leibniz Institute for Solar Physics (KIS) as a partnership contribution to the DKIST. The instrument is currently undergoing lab integration and is expected to be integrated into the DKIST coudé instrument laboratory at the end of 2021.

\subsection{Diffraction-Limited Near-Infrared Spectro-Polarimeter}

The DL-NIRSP is an integral-field, dual-beam spectro-polarimeter intended for studying magnetically sensitive spectral lines in the Sun's atmosphere with high spectral resolution 


\begin{tabular}{|c|c|c|c|c|c|c|c|}
\hline 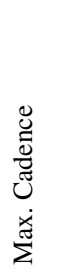 & 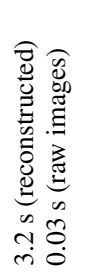 & 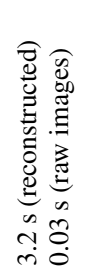 & 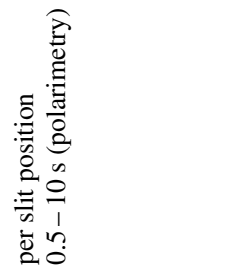 & 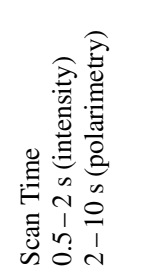 & 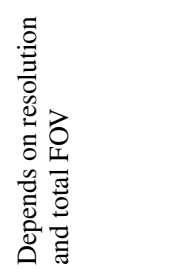 & 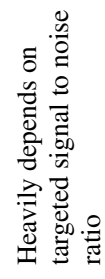 & 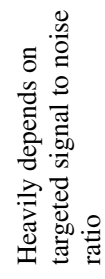 \\
\hline 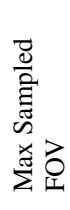 & 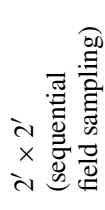 & 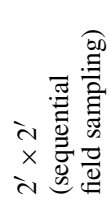 & 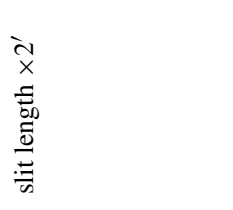 & 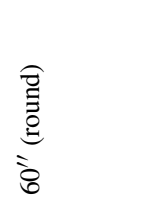 & 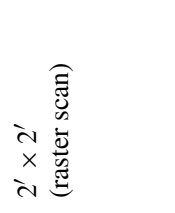 & 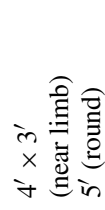 & 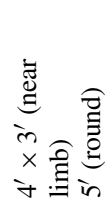 \\
\hline 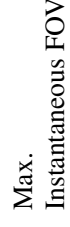 & $\begin{array}{l}i n \\
\text { in } \\
x \\
\text { in }\end{array}$ & $\begin{array}{l}\text { के } \\
x \\
\text { के }\end{array}$ & 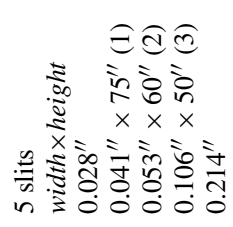 & 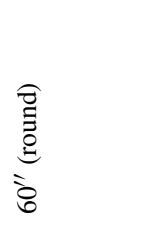 & 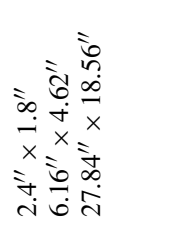 & 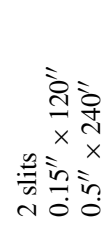 & $\begin{array}{l}\overline{8} \\
x \\
x \\
\overline{0}\end{array}$ \\
\hline 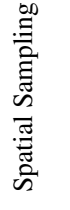 & 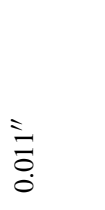 & $\begin{array}{l}\overline{1} \\
0 \\
0 \\
0\end{array}$ & 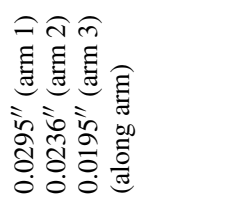 & 竞 & 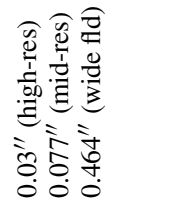 & 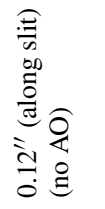 & 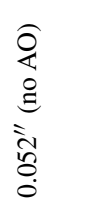 \\
\hline 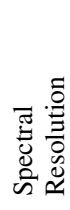 & $\begin{array}{l}8 \\
0 \\
0 \\
0 \\
1 \\
1 \\
2 \\
0\end{array}$ & $\begin{array}{l}8 \\
8 \\
\pm \\
1 \\
1 \\
8 \\
-1\end{array}$ & $\begin{array}{l}8 \\
8 \\
\infty \\
\infty \\
1\end{array}$ & 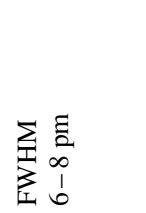 & $\begin{array}{l}8 \\
8 \\
\text { i }\end{array}$ & 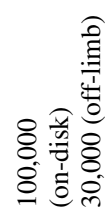 & $\underset{\mathrm{Z}}{\mathbb{Z}}$ \\
\hline 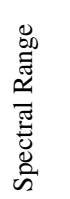 & 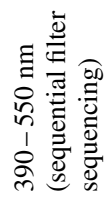 & 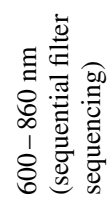 & 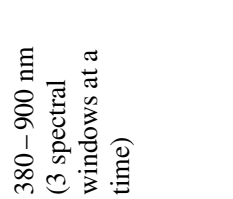 & 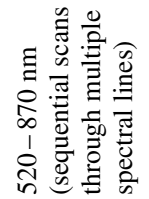 & 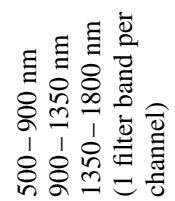 & 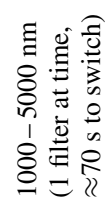 & $\begin{array}{l}1 \\
\vdots \\
8 \\
0 \\
1 \\
1 \\
8 \\
0\end{array}$ \\
\hline 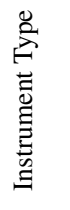 & 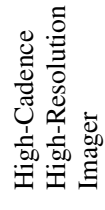 & 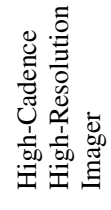 & 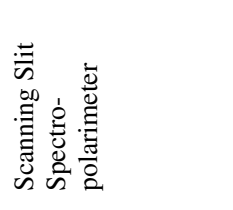 & 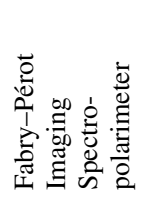 & 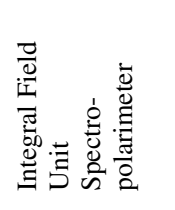 & 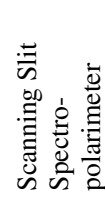 & 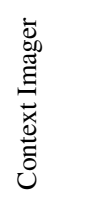 \\
\hline & 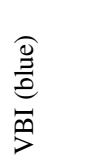 & $\overrightarrow{0}$ & $\stackrel{\tilde{n}}{>}$ & 5 & 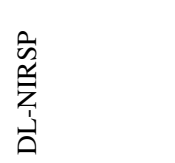 & 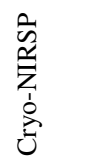 & 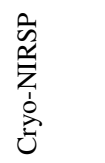 \\
\hline
\end{tabular}


$(125,000)$ and polarimetric accuracy. A novel fiber-optic integral-field unit, paired with selectable feed optics and a field-scanning mirror provide different spatial sampling ( 0.03 arcsec, 0.08 arcsec, and 0.5 arcsec). Multiple wavelengths can be observed simultaneously using three spectral arms to cover visible $(500-900 \mathrm{~nm})$ and infrared wavelengths $(900-$ $1350 \mathrm{~nm}$ and $1350-1800 \mathrm{~nm}$ ). DL-NIRSP's science objectives include measurements of the coronal magnetic field when DKIST is operated in coronagraphic mode. The instrument was designed and built by the University of Hawai'i's Institute for Astronomy (IfA). A detailed description of the instrument and first test results are given by Jaeggli et al. (2021).

\subsection{Cryogenic Near-Infrared Spectro-Polarimeter}

The primary goal of the CRYO-NIRSP is to measure the magnetic fields over a large FOV in the solar corona. The CRYO-NIRSP is designed to measure polarized light of coronal emission lines at infrared wavelength out to 5 microns. The CRYO-NIRSP has a large field of view ( 5 arcmin max) that can be sampled with a slit-scanning mechanism. By trading spatial and spectral resolution for enhanced signal-to-noise, coronal magnetic-field measurements using spectral lines such as in Fe XIII $(1075 \mathrm{~nm})$ and Si IX $(3935 \mathrm{~nm})$ can be performed with sufficient sensitivity. Depending on the specific target, observations will be performed at a spatial resolution of $1-2$ arcsec. Spectral resolution of up to $\mathrm{R}=100,000$ is achieved. The almost all-reflective design allows for high throughput. The spectrograph optics are cooled to cryogenic temperatures in order to reduce the thermal background allowing for mid-IR observations of the faint solar corona. CRYO-NIRSP provides the capability to perform on-disk observations at higher spatial resolution including at the important $\mathrm{CO}$ bands at $2333 \mathrm{~nm}$ and $4666 \mathrm{~nm}$. The University of Hawai'i IfA, in partnership with NSO, has led the development of the CRYO-NIRSP. Detailed descriptions of design, implementation, and first results are described by Kuhn et al. (2013), Fehlmann et al. (2016), and Fehlmann et al. (2021).

\subsection{Common Instrument Systems}

All instruments make use of common components and systems such as polarimetry calibration optics and mechanisms, camera systems and software systems, motion control, a data-handling system, and a timing and synchronization system. Avoiding duplication of effort and unnecessary complexity, and long-term maintainability were among the motivations for this approach. The approach requires up-front investment and careful planning of the overall instrumentation design effort. The Gregorian Optical Station provides common polarization-calibration retarders and modulation retarders covering the full wavelength range required by the visible and infrared polarimeters (Ferayorni et al., 2020). Instrumentpolarization calibration and analysis systems share common optical and mechanical components. A time-based timing and synchronization system (TRADS) is used to synchronize components, such as modulators, cameras, and motorized stages and instruments, such as the VBI red and blue channels (Ferayorni et al., 2014).

Facility camera systems were provided to instruments. The DL-NIRSP and CRYONIRSP utilize Teledyne $2 \mathrm{k} \times 2 \mathrm{k}$ Hawaii2-RG infrared detectors with custom-built electronics. DL-NIRSP uses short-wave IR detectors for two of its spectral arms. The CRYO-NIRSP uses mid-wave IR detectors for the spectrograph and short-wave IR for the context viewer. The $4 \mathrm{k} \times 4 \mathrm{k}$ visible wavelength sensors were developed by ANDOR and are deployed with the VBI, VTF, and DL-NIRSP. VISP uses three $2 \mathrm{k} \times 2 \mathrm{k}$ ANDOR ZYLA detectors. 
The Data Handling System (DHS) on the summit (not to be confused with the Boulder, Colorado-based data center) is used to collect, process, and display data from all instruments. The limited processing and display capabilities are primarily for quality-control purposes during observations. All cameras transfer data to the DHS via multiple camera lines. The DHS transfers data from the various instrument cameras using a high-speed network to the large-volume storage servers. High-speed camera lines are available, each capable of transmitting data at up to one gigabyte per second. The DHS provides quick-look displays and "sandbox processing" for more detailed quality control (Wampler and Goodrich, 2009; Cowan and Wampler, 2011). The DHS on the summit is the interface to the DKIST Data Center in Boulder for all instruments.

\section{Science Operations}

The effort involved in planning and implementing the science-operations processes and procedures for a complex facility such as DKIST is extensive. Complexity and effort are comparable to major night-time facilities and constitute an order of magnitude increase over previous solar facilities such as the DST. The DKIST operations plan provides service-mode observing, which, with the exception of experimental testing, was previously not available at ground-based solar telescopes. In order to achieve significantly higher observing efficiency, DKIST will perform the majority of observations in service mode. The traditional approach, which was implemented at the previous NSO facilities at Sacramento Peak and Kitt Peak, which afforded a principal investigator (PI) exclusive access to the observatory during a block of observing time, will become the exception. Flexible and dynamic scheduling of observations with the goal to optimally match solar conditions (target availability) and observing conditions (weather, seeing, etc.) are the key characteristics of service mode. Access time is granted when real-time guidance and direction from the PI and/or Co-Is is necessary and presence on site is required to perform the observations. In service mode, the observatory staff, on a daily basis, decides which approved and fully tested experiments are executed. The decision is guided by the Time Allocation Committee (TAC) provided ranking of observing proposals and how well proposal requirements match target availability and current observing conditions. The DKIST science operations are planned on a long-term (six months), medium-term (one to three months), and short-term (days to weeks) basis. The long-term planning is driven by the length of the solicitation cycle. The medium-term planning is driven by co-observing, access mode, coudé laboratory preparations (standard FIDO and DHS Configurations), and specific calibration planning. The short-term planning is driven by the Sun (solar changes) and weather conditions. Current solar ground-based observatories regularly support and co-observe with other observatories and space missions (coordinated observations). These co-observing efforts benefit science goals, specifically if additional wavelength information and/or temporal coverage is needed. It is expected that the DKIST will be requested to co-observe with other observatories or space missions on a regular basis. A comprehensive summary of how DKIST is operated including the proposal process, various proposal categories, experiment generation and execution, and tools available to support users as well as staff during the operations cycle is given by Tritschler et al. (2021).

\section{Data Center}

The DKIST Data Center represents a major transition in how the astronomy community users are granted access to facility data. In contrast to the previous national ground-based 
solar facilities, the DKIST program includes a data center that initially provides calibrated data to users. By making calibrated and eventually higher-level data products broadly available, the scientific utility and impact of DKIST is greatly enhanced. Data previously were in all practical terms only accessible to the PI who had taken the data during the PI-led observing run. The PI was also left to deal with the complex and time-consuming calibration effort necessary to arrive at science-ready data. The data center's mission includes:

i) Deliver quality-controlled, calibrated scientific data sets from data acquired by DKIST facility instruments utilized during the operational lifetime of DKIST

ii) Provide long-term management and curation of DKIST scientific data and metadata resulting from DKIST experiments

iii) Enable open, searchable, and documented access to data and metadata to a broad user base

iv) Develop and maintain software and hardware tools to support the acceptance, usage, and exploitation of DKIST data, accommodating changing scientific and operational needs inherent in a multi-decadal scientific program.

The Data Center thus is a facility that provides the crucial link between summit acquisition of science data and associated metadata, and its eventual use for enabling DKIST scientific goals. Berukoff et al. (2016) highlight key components and concepts that drive the design and implementation of the Data Center. DKIST is expected to deliver 3 PB of raw data per year. Calibrated data volumes are of similar magnitude. Raw and calibrated data as well as higher-level data products have to be curated over the lifetime of the observatory (two full Hale cycles). The complexity and operational flexibility of the five instruments on the coudé platform add to the data-handling challenge. Implementing automated calibration pipelines that remove instrumental and some atmospheric seeing effects constitutes the most significant challenges. Furthermore, the distribution of the large data sets in an efficient and reliable manner to a widely distributed user base and the required user support constitute significant technology challenges and operational effort. The data center is located at the NSO headquarters in Boulder, Colorado (Davey et al., 2021).

\section{First Light and First Results}

In December 2019 DKIST saw first solar light. First images of granulation were taken with the VBI red using the $789 \mathrm{~nm}$ and $705 \mathrm{~nm}$ filters of the VBI. The AO system was locked on granulation for the first time as well. The telescope was pointing close to Sun-center. It should be noted that neither all telescope thermal systems were fully operational nor was the $\mathrm{AO}$ working at its full capacity. $\mathrm{VBI} 4 \mathrm{k} \times 4 \mathrm{k}$ images were taken at a rate of $30 \mathrm{~Hz}$. Bursts of $80-100$ images were recorded with a cadence of three seconds. Several time sequences of speckle bursts of up to ten minutes were obtained over a period of three days. Wavefrontsensor telemetry was used to estimate seeing conditions during the first light observations. A Fried parameter of $r_{0}=6-7 \mathrm{~cm}$ was measured. As discussed in Section 5, significant local seeing may have been present and is reflected in the measured seeing parameter. Images were subjected to standard dark- and flat-field calibration. Bursts of 80 images each were used to derive speckle reconstructed images (Wöger, 2010). Noise filtering was applied to remove camera noise. The image, which was used for the first light press release in January 2020 and received broad coverage in the international press, is shown with Figure 15.

Figure 16 shows a comparison of a MHD simulated (MURaM) granulation image (courtesy M. Rempel) and a close-up of the observed granulation image displayed in Figure 15. 


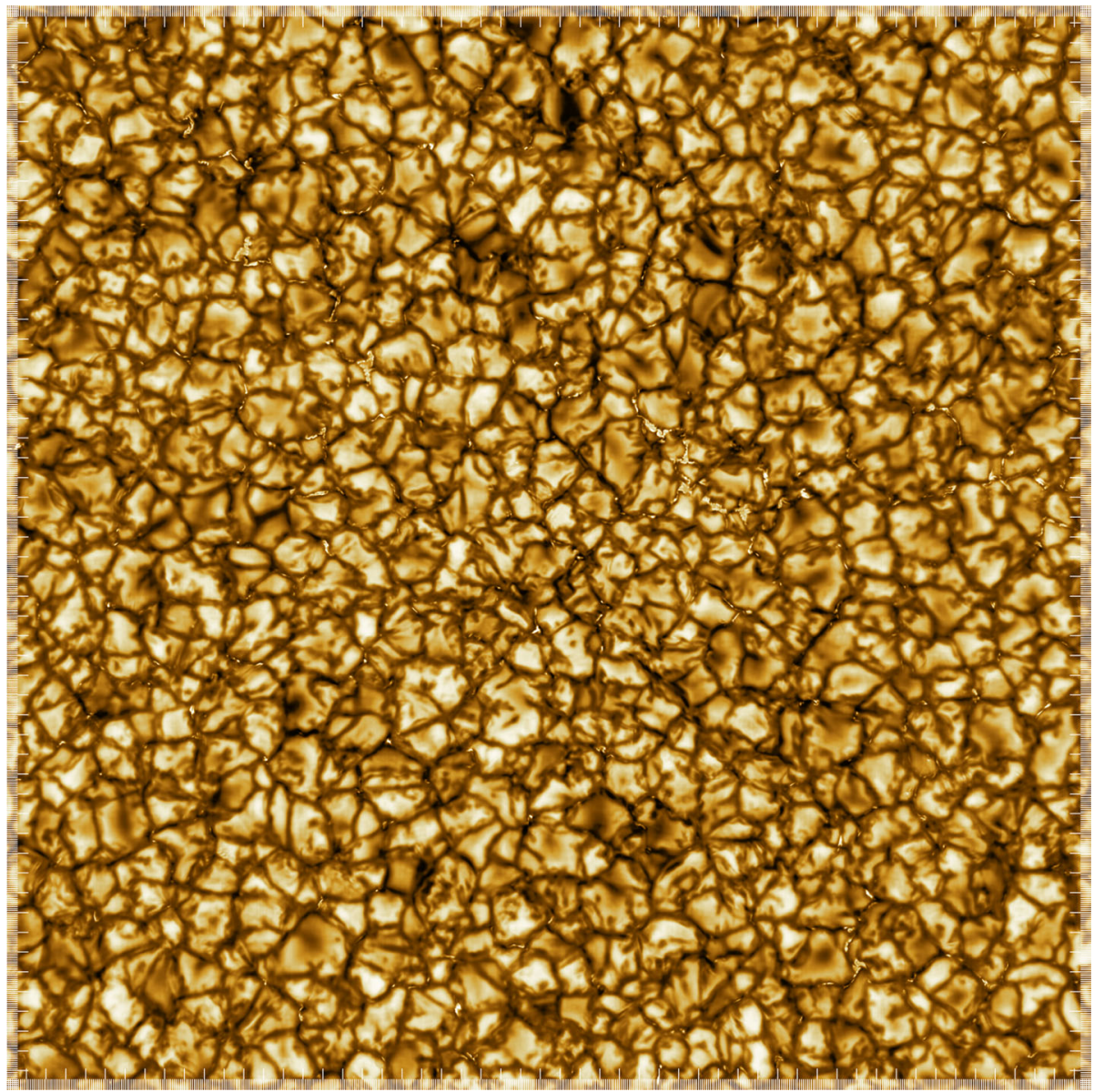

Figure 15 First-light image of granulation near Sun-center taken with VBI red. The FOV is $55 \operatorname{arcsec} \times 55$ arcsec (major tick marks are 1 arcsec). A burst of 80 short-exposure images was speckle reconstructed. The resolution achieved with this image is close to the diffraction limit at the wavelength of $789 \mathrm{~nm}$ $(0.04 \mathrm{arcsec})$. The image was processed to remove noise and enhance the visibility (contrast) of small-scale (magnetic) features. Wöger et al. (2021) present scientifically processed images that maintain photometric integrity.

This comparison demonstrates that with DKIST we now have resolution comparable to the resolution of highest-resolution MHD simulations. Substructure of what we might have called "bright points or sheets" is clearly visible in the observations. We emphasize that Figure 16 does not attempt or claim to verify MHD model predictions but merely demonstrates that DKIST provides a powerful tool to perform such model verification. As discussed in previous sections, detailed and quantitative verification of model predictions must be performed by, e.g., comparing modeled and observed physical parameters, such as velocities, magnetic-field strengths and directions, intensities, and their temporal evolution. The iterative process of verifying and improving models and model assumptions based on observations will occur over many years to come.

Figure 17 displays a continuum image at $789 \mathrm{~nm}$ and the simultaneously recorded $\mathrm{H} \alpha$ wing image. The $\mathrm{H} \alpha$ wing image shows small-scale fibril structure originating from the 
Figure 16 Qualitative comparison of DKIST first-light image (bottom) and MURaM simulated image (top) (MURaM: see Vögler et al., 2005). The simulation was provided by $\mathrm{M}$. Rempel and is a non-gray rerun at $8 \mathrm{~km}$ grid spacing of the simulation listed as "O16bM" in Table 1 of Rempel (2014). Major tick marks are 1 arcsec.
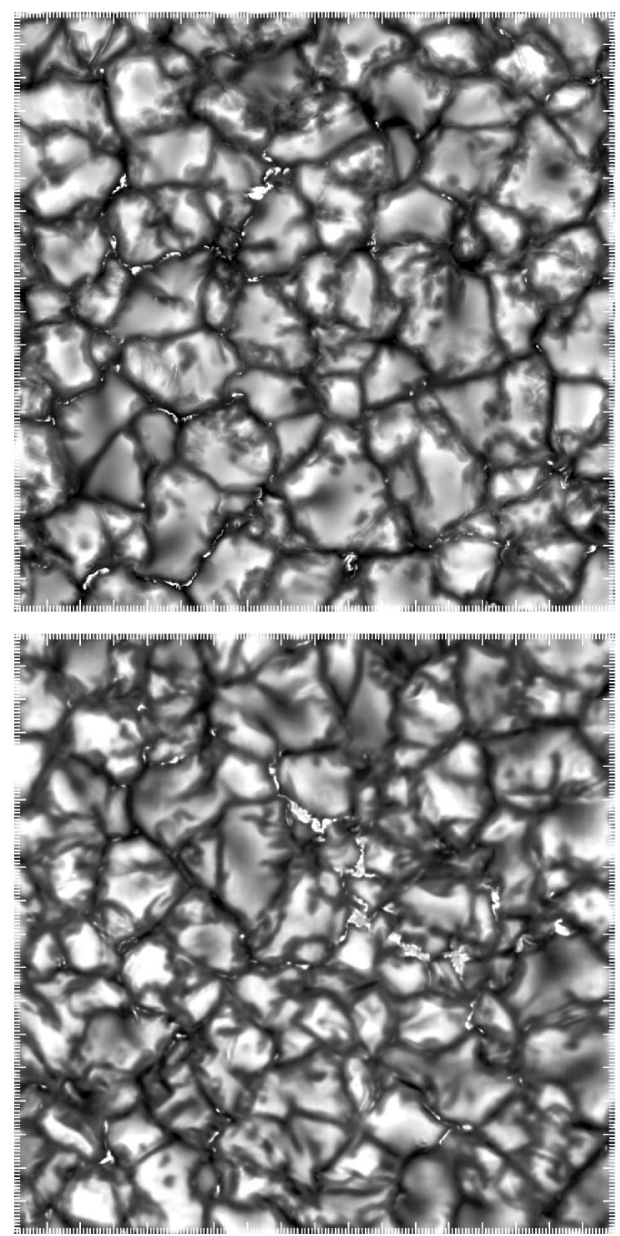

"bright points" seen in continuum. Short movies were recorded and reveal that these fibrils evolve on time scales of a few seconds.

Figure 18 shows the first image of a sunspot from DKIST. The image was obtained during the commissioning phase with the context viewer of the wavefront correction system. The broadband image was recorded at visible wavelengths $(530 \mathrm{~nm})$ and contains spatial structure near the diffraction limit. Penumbral and umbral dark cores (Langhans et al., 2007) are clearly resolved. The movie linked to Figure 18 shows the dynamic evolution of sunspot fine-structure over a short period of time (about 100 seconds). The sequence of diffraction-limited, broadband images was recorded with the context viewer of the DKIST adaptive-optics system at a wavelength of $530 \mathrm{~nm}$. The movie reveals a wealth of quickly evolving fine-structure within the spot's umbra, penumbra, and surrounding quiet Sun. For example, narrow $\left(<0.1^{\prime \prime}\right)$, dark lanes are observed consistently in both umbral dots (UD) and penumbral grains (PG). A few typical examples are pointed out with arrows. Narrow, dark lanes within bright UDs and PGs have been predicted by numerical simulations of magnetoconvection and are a consequence of strong upflow plumes in areas of lower magneticfield strength (Schüssler and Vögler, 2006). The dark lanes evolve significantly during 100 

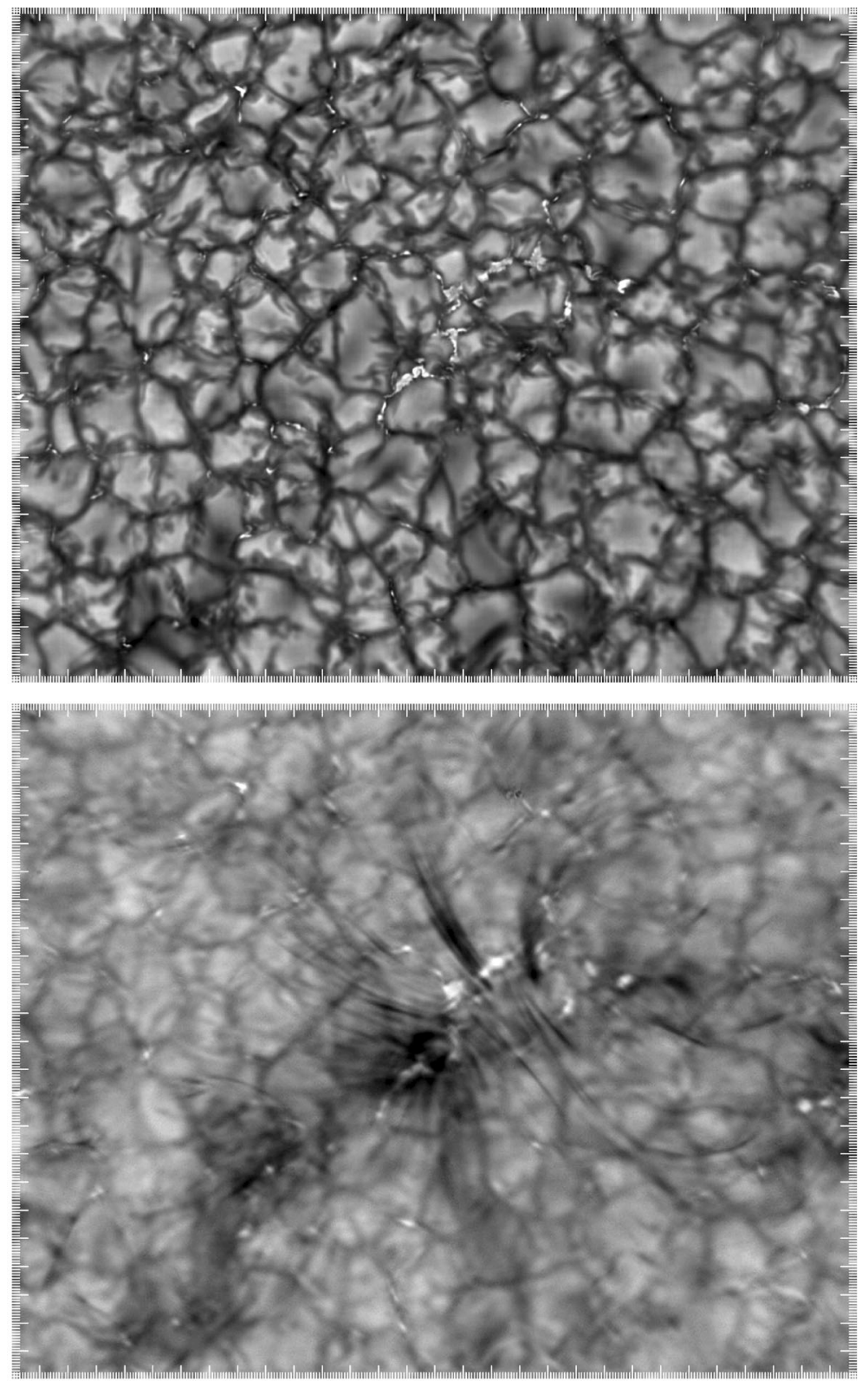

Figure 17 (Upper) Continuum image at $789 \mathrm{~nm}$ and (Lower) simultaneous $\mathrm{H} \alpha$ wing image. The filter is $0.05 \mathrm{~nm}$ wide with its pass-band centered on the red wing of the $\mathrm{H} \alpha$ line. Strong, likely field-aligned chromospheric flows appear as dark fibrils. The $\mathrm{H} \alpha$ wing image shows fibril structure originating from the "bright points" seen in continuum. These fibrils evolve on time scales of a few seconds. "Print-through" of the photospheric granulation intensity pattern is visible, but at much reduced contrast. Major (minor) tick marks are $1 \operatorname{arcsec}(0.1 \operatorname{arcsec})$ apart. 

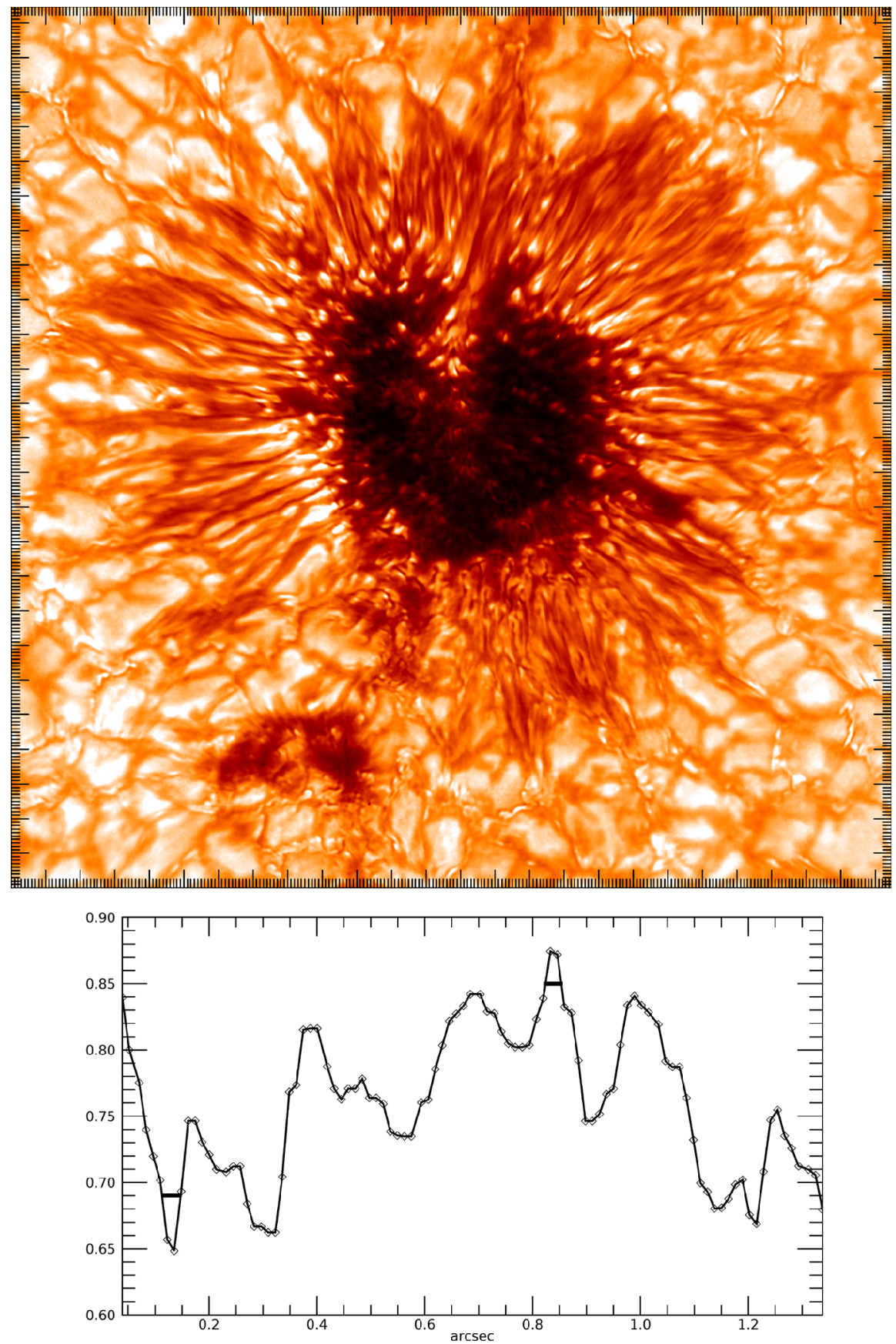

Figure 18 (Top) First DKIST image of a sunspot. This speckle-reconstructed image was recorded with the $2 \mathrm{k} \times 2 \mathrm{k}$ WFC context viewer at the wavelength of $530 \mathrm{~nm}$. The FOV is approximately $25 \operatorname{arcsec} \times 25 \operatorname{arcsec}$. Major (minor) tick marks are 1 arcsec $(0.1 \mathrm{arcsec})$ apart. The sunspot was located at a center-to-limb distance of 0.45 . (Bottom) Trace across penumbral filaments that demonstrates that the image achieves resolution close to the diffraction limit at $530 \mathrm{~nm}$. The horizontal lines plotted across two selected penumbral filaments are of width 0.027 arcsec, i.e. the diffraction limit at $530 \mathrm{~nm}$. A short movie is available as electronic supplemental material. 
seconds giving the impression of small-scale, overturning convection occurring in these features. DKIST's spectro-polarimeters will allow detailed analysis of these small-scale feature and comparison to model predictions.

Additional first-light VBI images and movies, including first quantitative analysis, are presented by Wöger et al. (2021).

\section{Summary}

After more than two decades, the $4 \mathrm{~m}$ DKIST has finally come to fruition. First results demonstrate that DKIST is achieving its design requirements, which were defined by the solar community as represented by the science working group. First imagery clearly shows the potential of DKIST and its set of state-of-the-art instruments to revolutionize solar astronomy. The DKIST design represents an excellent balance of various design drivers that allows the pursuit of the many pressing science drivers by providing the most capable solar telescope facility built to date. Many technical challenges had to be addressed and difficult trades had to be performed to achieve this goal. Operations of DKIST are ramping up. The first proposal call for the Operations Commissioning Phase has been released. The DKIST data center will serve several petabytes per year of calibrated data to the world-wide user community. Higher-level data products such as inversions will be added as resources allow. As the DKIST data base builds up over time, many novel science projects will emerge from the vast amount of available data. The scientific exploitation is just beginning and DKIST will significantly advance our knowledge of the Sun over the next several decades.

Acknowledgments The research reported herein is based in part on data collected with the Daniel K. Inouye Solar Telescope (DKIST), a facility of the National Solar Observatory (NSO). NSO is managed by the Association of Universities for Research in Astronomy, Inc., and is funded by the National Science Foundation. Any opinions, findings and conclusions or recommendations expressed in this publication are those of the authors and do not necessarily reflect the views of the National Science Foundation or the Association of Universities for Research in Astronomy, Inc. DKIST is located on land of spiritual and cultural significance to Native Hawai'ian people. The use of this important site to further scientific knowledge is done so with appreciation and respect.

Funding Note Open Access funding provided by NSO. The National Solar Observatory (NSO) is operated by the Association of Universities for Research in Astronomy, Inc. (AURA), under cooperative agreement with the National Science Foundation.

Disclosure of Potential Conflicts of Interest The authors declare that they have no conflicts of interest.

Publisher's Note Springer Nature remains neutral with regard to jurisdictional claims in published maps and institutional affiliations.

Open Access This article is licensed under a Creative Commons Attribution 4.0 International License, which permits use, sharing, adaptation, distribution and reproduction in any medium or format, as long as you give appropriate credit to the original author(s) and the source, provide a link to the Creative Commons licence, and indicate if changes were made. The images or other third party material in this article are included in the article's Creative Commons licence, unless indicated otherwise in a credit line to the material. If material is not included in the article's Creative Commons licence and your intended use is not permitted by statutory regulation or exceeds the permitted use, you will need to obtain permission directly from the copyright holder. To view a copy of this licence, visit http://creativecommons.org/licenses/by/4.0/. 


\section{References}

Avrett, E.H.: 2003, The solar temperature minimum and chromosphere. In: Pevtsov, A.A., Uitenbroek, H. (eds.) Current Theoretical Models and Future High Resolution Solar Observations: Preparing for ATST CS-286, Astron. Soc. Pacific, San Francisco, 419. ADS.

Bailey, J., Lucas, P.W., Hough, J.H.: 2010, The linear polarization of nearby bright stars measured at the parts per million level. Mon. Not. Roy. Astron. Soc. 405, 2570. DOI. ADS.

Bailey, J., Ulanowski, Z., Lucas, P.W., Hough, J.H., Hirst, E., Tamura, M.: 2008, The effect of airborne dust on astronomical polarization measurements. Mon. Not. Roy. Astron. Soc. 386, 1016. DOI. ADS.

Beckers, J.M.: 1995, CLEAR: A concept for a coronagraph and low emissivity astronomical reflector. In: Kuhn, J.R., Penn, M.J. (eds.) Infrared Tools for Solar Astrophysics: What's Next? Proc. Fifteenth National Solar Observatory/Sacramento Peak Summer Workshop, World Scientific, Singapore, 145. ADS.

Beckers, J.M., NSO Staff: 1997, Progress report on a feasibility study of a large optical/infrared solar telescope (CLEAR). In: Schmieder, B., del Toro Iniesta, J.C., Vazquez, M. (eds.) 1st Advances in Solar Physics Euroconference. Advances in Physics of Sunspots CS-118, Astron. Soc. Pacific, San Francisco, 340. ADS.

Beckers, J.: 2002, Daytime seeing measurements for the Advanced Technology Solar Telescope. In: Vernin, J., Benkhaldoun, Z., Muñoz-Tuñón, C. (eds.) Astronomical Site Evaluation in the Visible and Radio Range CS-266, Astron. Soc. Pacific, San Francisco, 350. ADS.

Bellot Rubio, L., Orozco Suárez, D.: 2019, Quiet Sun magnetic fields: An observational view. Liv. Rev. Solar Phys. 16, 1. DOI. ADS.

Berger, T., Reardon, K., Elmore, D., Woeger, F., Tritschler, A., Rimmele, T.: 2014, Future diagnostic capabilities: The 4-meter Daniel K. Inouye solar telescope. In: 40th COSPAR Scientific Assembly 40, E2.2. ADS.

Berukoff, S., Hays, T., Reardon, K., Spiess, D., Watson, F., Wiant, S.: 2016, Petascale cyberinfrastructure for ground-based solar physics: Approach of the DKIST data center. In: Chiozzi, G., Guzman, J.C. (eds.) Software and Cyberinfrastructure for Astronomy IV, Proc. Soc. Photo-Opt. Instrum. Eng. (SPIE) CS-9913, 99131F. DOI. ADS.

Bianda, M., Ramelli, R., Gisler, D.: 2009, Observing the second solar spectrum at IRSOL. In: Berdyugina, S.V., Nagendra, K.N., Ramelli, R. (eds.) Solar Polarization 5: In Honor of Jan Stenflo CS-405, Astron. Soc. Pacific, San Francisco, 17. ADS.

Bianda, M., Stenflo, J.O., Solanki, S.K.: 1998, Hanle diagnostics of solar magnetic fields: The Sr II 4078 Angstrom line. Astron. Astrophys. 337, 565 ADS.

Bjørgen, J.P., Leenaarts, J., Rempel, M., Cheung, M.C.M., Danilovic, S., de la Cruz Rodríguez, J., Sukhorukov, A.V.: 2019, Three-dimensional modeling of chromospheric spectral lines in a simulated active region. Astron. Astrophys. 631, A33. DOI. ADS.

Cameron, R., Schüssler, M.: 2015, The crucial role of surface magnetic fields for the solar dynamo. Science 347, 1333. DOI. ADS.

Canzian, B., Barentine, J., Arendt, J., Bader, S., Danyo, G., Heller, C.: 2012, Progress making the top end optical assembly (TEOA) for the 4-meter Advanced Technology Solar Telescope. In: Stepp, L.M., Gilmozzi, R., Hall, H.J. (eds.) Ground-Based and Airborne Telescopes IV, Proc. Soc. Photo-Opt. Instrum. Eng. (SPIE) CS-8444, 844409. DOI. ADS.

Cavaco, J., Wirth, A.: 2014, Deformable mirror designs for extreme AO (XAO). In: Marchetti, E., Close, L.M., Véran, J.-P. (eds.) Adaptive Optics Systems IV, Proc. Soc. Photo-Opt. Instrum. Eng. (SPIE) CS9148, 914823. DOI. ADS.

Cheung, M.C.M., Rempel, M., Chintzoglou, G., Chen, F., Testa, P., Martínez-Sykora, J., Sainz Dalda, A., DeRosa, M.L., Malanushenko, A., Hansteen, V., De Pontieu, B., Carlsson, M., Gudiksen, B., McIntosh, S.W.: 2019, A comprehensive three-dimensional radiative magnetohydrodynamic simulation of a solar flare. Nat. Astron. 3, 160. DOI. ADS.

Cho, M.K., DeVries, J., Hansen, E.: 2007, Thermal performance of the ATST secondary mirror. In: Zhang, Y., Jiang, W., Cho, M.K. (eds.) 3rd International Symposium on Advanced Optical Manufacturing and Testing Technologies: Large Mirrors and Telescopes, Proc. Soc. Photo-Opt. Instrum. Eng. (SPIE) CS6721, 672102. DOI. ADS.

Cho, M.K., Price, R.S., Moon, I.K.: 2006, Optimization of the ATST primary mirror support system. In: Atad-Ettedgui, E., Antebi, J., Lemke, D. (eds.) Optomechanical Technologies for Astronomy, Proc. Soc. Photo-Opt. Instrum. Eng. (SPIE) CS-6273, 62731E. DOI. ADS.

Collados, M., Bettonvil, F., Cavaller, L., Ermolli, I., Gelly, B., Pérez, A., Socas-Navarro, H., Soltau, D., Volkmer, R. (EST Team): 2013, The European Solar Telescope. Mem. Soc. Astron. Ital. 84, 379. ADS.

Cowan, B., Wampler, S.: 2011, Technologies for high speed data handling in the ATST. In: Evans, I.N., Accomazzi, A., Mink, D.J., Rots, A.H. (eds.) Astronomical Data Analysis Software and Systems XX CS-442, Astron. Soc. Pacific, San Francisco, 297. ADS. 
Craig, S., Hansen, E., Hubbard, R.P., Kneale, R.: 2014, Daniel K. Inouye Solar Telescope systems engineering update. In: Angeli, G.Z., Dierickx, P. (eds.) Modeling, Systems Engineering, and Project Management for Astronomy VI, Proc. Soc. Photo-Opt. Instrum. Eng. (SPIE) CS-9150, 915019. DOI. ADS.

Dalrymple, N.E., Oschmann, J.M. Jr., Hubbard, R.P.: 2004, ATST enclosure: Seeing performance, thermal modeling, and error budgets. In: Craig, S.C., Cullum, M.J. (eds.) Modeling and Systems Engineering for Astronomy, Proc. Soc. Photo-Opt. Instrum. Eng. (SPIE) CS-5497, 497. DOI. ADS.

Davey, A., et al.: 2021, The DKIST Data Center. Solar Phys. 296, in preparation.

de Gouveia Dal Pino, E.M.: 2011, Cosmic magnetic fields: From stars and galaxies to the primordial universe. In: Perez Berliaffa, S.E., Novello, M., Ruffini, R. (eds.) The Sun, the Stars, the Universe and General Relativity, Cambridge Sci. Pub., Cambridge, 37. ADS.

de Wijn, A.G., Stenflo, J.O., Solanki, S.K., Tsuneta, S.: 2009, Small-scale solar magnetic fields. Space Sci. Rev. 144, 275. DOI. ADS.

de Wijn, A.G., et al.: 2021, The DKIST visible spectro polarimeter. Solar Phys. 296, in preparation.

Deng, Y., Liu, Z., Qu, Z., Liu, Y., Ji, H.: 2016, The Chinese giant solar telescope. In: Dorotovic, I., Fischer, C.E., Temmer, M. (eds.) Coimbra Solar Physics Meeting: Ground-Based Solar Observations in the Space Instrumentation Era CS-504, Astron. Soc. Pacific, San Francisco, 293. ADS.

Derks, A., Beck, C., Martínez Pillet, V.: 2018, Inferring telescope polarization properties through spectral lines without linear polarization. Astron. Astrophys. 615, A22. DOI.

Dima, G.I., Kuhn, J.R., Schad, T.A.: 2019, Coronagraphic observations of Si X $\lambda 14301$ and Fe XIII $\lambda 10747$ linearly polarized spectra using the SOLARC telescope. Astrophys. J. 877, 144. DOI. ADS.

Dreyer, O., Ippa, A., Seubert, S., Kärcher, H.J., Jeffers, P., Bonomi, G.: 2014, Performance verification of the DKIST Mount and Coudé Laboratory. In: Stepp, L.M., Gilmozzi, R., Hall, H.J. (eds.) Ground-Based and Airborne Telescopes V, Proc. Soc. Photo-Opt. Instrum. Eng. (SPIE) CS-9145, 91452A. DOI. ADS.

Elmore, D.F., Lin, H., Socas-Navarro, H., Jaeggli, S.A.: 2010, Utilization of redundant polarized solar spectra to infer the polarization properties of the new generation of large aperture solar telescopes. In: McLean, I.S., Ramsay, S.K., Takami, H. (eds.) Ground-Based and Airborne Instrumentation for Astronomy III, Proc. Soc. Photo-Opt. Instrum. Eng. (SPIE) CS-7735, 77354E. DOI. ADS.

Elmore, D.F., Rimmele, T., Casini, R., Hegwer, S., Kuhn, J., Lin, H., McMullin, J.P., Reardon, K., Schmidt, W., Tritschler, A., Wöger, F.: 2014, The Daniel K. Inouye Solar Telescope first light instruments and critical science plan. In: Ramsay, S.K., McLean, I.S., Takami, H. (eds.) Ground-Based and Airborne Instrumentation for Astronomy V, Proc. Soc. Photo-Opt. Instrum. Eng. (SPIE) CS-9147, 914707. DOI. ADS.

Engvold, O.: 1991, Large Earth-based Solar Telescope-LEST. Adv. Space Res. 11, 157. DOI. ADS.

Fan, Y., Gibson, S.E.: 2004, Numerical simulations of three-dimensional coronal magnetic fields resulting from the emergence of twisted magnetic flux tubes. Astrophys. J. 609, 1123. DOI. ADS.

Fan, Y., Gibson, S., Tomczyk, S.: 2018, The eruption of a prominence-carrying coronal flux rope: Forward synthesis of the magnetic field strength measurement by the COronal Solar Magnetism Observatory large coronagraph. Astrophys. J. 866, 57. DOI. ADS.

Fehlmann, A., Giebink, C., Kuhn, J.R., Messersmith, E.J., Mickey, D.L., Scholl, I.F., James, D., Hnat, K., Schickling, G., Schickling, R.: 2016, Cryogenic near infrared spectropolarimeter for the Daniel K. Inouye Solar Telescope. In: Evans, C.J., Simard, L., Takami, H. (eds.) Ground-Based and Airborne Instrumentation for Astronomy VI, Proc. Soc. Photo-Opt. Instrum. Eng. (SPIE) CS-9908, 99084D. DOI. ADS.

Fehlmann, A., et al.: 2021, The DKIST cryogenic near-infrared spectro polarimeter. Solar Phys. 296, in preparation.

Ferayorni, A., Beard, A., Berst, C., Goodrich, B.: 2014, DKIST controls model for synchronization of instrument cameras, polarization modulators, and mechanisms. In: Chiozzi, G., Radziwill, N.M. (eds.) Software and Cyberinfrastructure for Astronomy III, Proc. Soc. Photo-Opt. Instrum. Eng. (SPIE) CS9152, 91520Z. DOI. ADS.

Ferayorni, A., Runyan, C., Gregory, S., Kootz, A., Rimmele, L., Sueoka, S., Harrington, D.: 2020, Commissioning of the Gregorian optical system calibration unit for DKIST. In: Proc. Soc. Photo-Opt. Instrum. Eng. (SPIE), 11450-108. In press.

Fossati, L., Bagnulo, S., Mason, E., Landi Degl'Innocenti, E.: 2007, Standard stars for linear polarization observed with FORS1. In: Sterken, C. (ed.) The Future of Photometric, Spectrophotometric and Polarimetric Standardization CP-364, Astron. Soc. Pacific, San Francisco, 503. ADS.

Gibson, S.E., Fan, Y.: 2006, Coronal prominence structure and dynamics: A magnetic flux rope interpretation. J. Geophys. Res. 111, A12103. DOI. ADS.

Goode, P.R., Cao, W.: 2012a, The 1.6 m off-axis New Solar Telescope (NST) in Big Bear. In: Stepp, L.M., Gilmozzi, R., Hall, H.J. (eds.) Ground-Based and Airborne Telescopes IV, Proc. Soc. Photo-Opt. Instrum. Eng. (SPIE) CS-8444, 844403. DOI. ADS. 
Goode, P.R., Cao, W.: 2012b, The 1.6 m off-axis New Solar Telescope (NST) in Big Bear. In: Rimmele, T.R., Tritschler, A., Wöger, F., Collados Vera, M., Socas-Navarro, H., Schlichenmaier, R., Carlsson, M., Berger, T., Cadavid, A., Gilbert, P.R., Goode, P.R., Knölker, M. (eds.) Second ATST-EAST Meeting: Magnetic Fields from the Photosphere to the Corona CS-463, Astron. Soc. Pacific, San Francisco, 357. ADS.

Goodrich, B.D., Wampler, S.B.: 2004, Software controls for the ATST Solar Telescope. In: Lewis, H., Raffi, G. (eds.) Advanced Software, Control, and Communication Systems for Astronomy, Proc. Soc. PhotoOpt. Instrum. Eng. (SPIE) CS-5496, 518. DOI. ADS.

Gorman, M., Galapon, C., Montijo, G., Phelps, L., Murga, G.: 2016, Cooling a solar telescope enclosure: Plate coil thermal analysis. In: Angeli, G.Z., Dierickx, P. (eds.) Modeling, Systems Engineering, and Project Management for Astronomy VII, Proc. Soc. Photo-Opt. Instrum. Eng. (SPIE) CS-9911, 99111U. DOI. ADS.

Grigoryev, V., Demidov, M., Kolobov, D., Pulyaev, V., Skomorovsky, V., Chuprakov, S.: 2020, Project of the Large Solar Telescope with mirror $3 \mathrm{~m}$ in diameter. J. Solar-Terr. Phys. 6, 14. DOI. ADS.

Hansen, E., Bulau, S., Phelps, L.: 2008, Advanced Technology Solar Telescope M1 thermal control system design, modeling, and prototype testing. In: Stepp, L.M., Gilmozzi, R. (eds.) Ground-Based and Airborne Telescopes II, Proc. Soc. Photo-Opt. Instrum. Eng. (SPIE) CS-7012, 701233. DOI. ADS.

Hansen, E., Price, R., Hubbard, R.: 2006, Advanced Technology Solar Telescope optical design. In: Stepp, L.M. (ed.) Ground-Based and Airborne Telescopes, Proc. Soc. Photo-Opt. Instrum. Eng. (SPIE) CS6267, 62673Z. DOI. ADS.

Harrington, D.M., Kuhn, J.R., Ariste, A.L.: 2017, Daytime sky polarization calibration limitations. J. Astron. Telesc. Instrum. Syst. 3, 018001. DOI.

Harrington, D.M., Kuhn, J.R., Hall, S.: 2011, Deriving telescope Mueller matrices using daytime sky polarization observations. Publ. Astron. Soc. Pac. 123, 799. DOI. ADS.

Harrington, D., Kuhn, J.R., Nevin, R.: 2015, Calibrating and stabilizing spectropolarimeters with charge shuffling and daytime sky measurements. Astron. Astrophys. 578, A126. DOI.

Harrington, D.M., Sueoka, S.R.: 2017, Polarization modeling and predictions for Daniel K. Inouye Solar Telescope part 1: Telescope and example instrument configurations. J. Astron. Telesc. Instrum. Syst. $\mathbf{3}$, 018002. DOI. ADS.

Harrington, D.M., Sueoka, S.R.: 2018a, Polarization modeling and predictions for Daniel K. Inouye Solar Telescope part 4: Calibration accuracy over field of view, retardance spatial uniformity, and achromat design sensitivity. J. Astron. Telesc. Instrum. Syst. 4, 044006. DOI. ADS.

Harrington, D.M., Sueoka, S.R.: 2018b, Polarization modeling and predictions for DKIST part 3: Focal ratio and thermal dependencies of spectral polarization fringes and optic retardance. J. Astron. Telesc. Instrum. Syst. 4, 018006. DOI. ADS.

Harrington, D.M., Sueoka, S.R., White, A.J.: 2019, Polarization modeling and predictions for Daniel K. Inouye Solar Telescope part 5: Impacts of enhanced mirror and dichroic coatings on system polarization calibration. J. Astron. Telesc. Instrum. Syst. 5, 038001. DOI. ADS.

Harrington, D.M., Berdyugina, S.V., Kuzmychov, O., Kuhn, J.R.: 2015, Correcting systematic polarization effects in Keck LRISp spectropolarimetry to 0.05 percent. Publ. Astron. Soc. Pac. 127, 757. DOI.

Harrington, D.M., Snik, F., Keller, C.U., Sueoka, S.R., van Harten, G.: 2017, Polarization modeling and predictions for DKIST part 2: Application of the Berreman calculus to spectral polarization fringes of beamsplitters and crystal retarders. J. Astron. Telesc. Instrum. Syst. 3, 048001. DOI. ADS.

Harrington, D.M., Jaeggli, S., Schad, T., White, A.J., Sueoka, S.R.: 2020a, Polarization modeling and predictions for Daniel K. Inouye Solar Telescope part 6: Fringe mitigation with polycarbonate modulators and optical contact calibration retarders. J. Astron. Telesc. Instrum. Syst. 6, 038001. DOI.

Harrington, D.M., Jaeggli, S., Schad, T., White, A.J., Sueoka, S.R.: 2020b, Polarization modeling and predictions for Daniel K. Inouye Solar Telescope part 7. J. Astron. Telesc., Instrum., Syst. 7, submitted.

Harrington, D.M., et al.: 2021, Polarimetry with DKIST. Solar Phys. 296, in preparation.

Hasan, S.S.: 2012, India's national large solar telescope. In: Rimmele, T.R., Tritschler, A., Wöger, F., Collados Vera, M., Socas-Navarro, H., Schlichenmaier, R., Carlsson, M., Berger, T., Cadavid, A., Gilbert, P.R., Goode, P.R., Knölker, M. (eds.) Second ATST-EAST Meeting: Magnetic Fields from the Photosphere to the Corona CP-463, Astron. Soc. Pacific, San Francisco, 395. ADS.

Hill, F., Beckers, J., Brandt, P., Briggs, J., Brown, T., Brown, W., Collados, M., Denker, C., Fletcher, S., Hegwer, S., Horst, T., Komsa, M., Kuhn, J., Lecinski, A., Lin, H., Oncley, S., Penn, M., Rimmele, T.R., Socas-Navarro, H., Streander, K.: 2004, Solar site testing for the Advanced Technology Solar Telescope. In: Oschmann, J.M. Jr. (ed.) Ground-Based Telescopes, Proc. Soc. Photo-Opt. Instrum. Eng. (SPIE) CS-5489, 122. DOI. ADS.

Hill, F., Beckers, J., Brandt, P., Briggs, J., Brown, T., Brown, W., Collados, M., Denker, C., Fletcher, S., Hegwer, S., Horst, T., Komsa, M., Kuhn, J., Lecinski, A., Lin, H., Oncley, S., Penn, M., Radick, R., Rimmele, T., Socas-Navarro, H., Streander, K.: 2006, Site testing for the Advanced Technology Solar 
Telescope. In: Stepp, L.M. (ed.) Ground-Based and Airborne Telescopes, Proc. Soc. Photo-Opt. Instrum. Eng. (SPIE) CS-6267, 62671T. DOI. ADS.

Hough, J.H., Lucas, P.W., Bailey, J.A., Tamura, M., Hirst, E., Harrison, D., Bartholomew-Biggs, M.: 2006, PlanetPol: A very high sensitivity polarimeter CS-118, 1302. DOI. ADS.

Hubbard, R., Craig, S., Kneale, R.: 2016, Daniel K. Inouye Solar Telescope systems engineering update. In: Angeli, G.Z., Dierickx, P. (eds.) Modeling, Systems Engineering, and Project Management for Astronomy VII, Proc. Soc. Photo-Opt. Instrum. Eng. (SPIE) CS-9911, 99112F. DOI. ADS.

Hubbard, J., Goodrich, B., Wampler, S.: 2010, The ATST base: Command-action-response in action. In: Radziwill, N.M., Bridger, A. (eds.) Software and Cyberinfrastructure for Astronomy, Proc. Soc. PhotoOpt. Instrum. Eng. (SPIE) CS-7740, 77402R. DOI. ADS.

Hubbard, R.P., Oschmann, J.M. Jr.: 2004, Monte Carlo telescope performance modeling. In: Craig, S.C., Cullum, M.J. (eds.) Modeling and Systems Engineering for Astronomy, Proc. Soc. Photo-Opt. Instrum. Eng. (SPIE) CS-5497, 129. DOI. ADS.

Isobe, H., Proctor, M.R.E., Weiss, N.O.: 2008, Convection-driven emergence of small-scale magnetic fields and their role in coronal heating and solar wind acceleration. Astrophys. J. Lett. 679, L57. DOI. ADS.

Jaeggli, S., et al.: 2021, The DKIST diffraction-limited near-infrared spectro polarimeter. Solar Phys. 296, in preparation.

Jedamzik, R., Werner, T., Westerhoff, T.: 2014, Production of the $4.26 \mathrm{~m}$ ZERODUR mirror blank for the Advanced Technology Solar telescope (ATST). In: Navarro, R., Cunningham, C.R., Barto, A.A. (eds.) Advances in Optical and Mechanical Technologies for Telescopes and Instrumentation, Proc. Soc. PhotoOpt. Instrum. Eng. (SPIE) CS-9151, 915131. DOI. ADS.

Jeffers, P., Manuel, E., Dreyer, O., Kärcher, H.: 2012a, ATST telescope pier. In: Stepp, L.M., Gilmozzi, R., Hall, H.J. (eds.) Ground-Based and Airborne Telescopes IV, Proc. Soc. Photo-Opt. Instrum. Eng. (SPIE) CS-8444, 84440L. DOI. ADS.

Jeffers, P., Stolz, G., Bonomi, G., Dreyer, O., Kärcher, H.: 2012b, ATST telescope mount: Telescope of machine tool. In: Stepp, L.M., Gilmozzi, R., Hall, H.J. (eds.) Ground-Based and Airborne Telescopes IV, Proc. Soc. Photo-Opt. Instrum. Eng. (SPIE) CS-8444, 84443T. DOI. ADS.

Jeffers, P., Warner, M., Craig, S., Hubbard, R., Marshall, H.: 2012c, Transitioning from conceptual design to construction performance specification. In: Modeling, Systems Engineering, and Project Management for Astronomy V, Society of Photo-Optical Instrumentation Engineers (SPIE) CS-8449, 84490B. DOI. ADS.

Jeffers, P., Trieloff, T., Kärcher, H., Seubert, S., McBride, W.: 2016, DKIST telescope mount factory testing overview and lessons learned. In: Hall, H.J., Gilmozzi, R., Marshall, H.K. (eds.) Ground-Based and Airborne Telescopes VI, Proc. Soc. Photo-Opt. Instrum. Eng. (SPIE) CS-9906, 99061E. DOI. ADS.

Jennings, D.E., Deming, D., McCabe, G., Sada, P.V., Moran, T.: 2002, Solar magnetic field studies using the 12 micron emission lines. IV. Observations of a delta region solar flare. Astrophys. J. 568, 1043. DOI. ADS.

Johansson, E.M., Goodrich, B.: 2012, Simultaneous control of multiple instruments at the Advanced Technology Solar Telescope. In: Radziwill, N.M., Chiozzi, G. (eds.) Software and Cyberinfrastructure for Astronomy II, Proc. Soc. Photo-Opt. Instrum. Eng. (SPIE) CS-8451, 84510J. DOI. ADS.

Johansson, E., Cummings, K., Drobilek, M., Johnson, L., Richards, K., Rampy, R., Wöger, F.: 2018, The DKIST low order wavefront sensor. In: Close, L.M., Schreiber, L., Schmidt, D. (eds.) Adaptive Optics Systems VI, Proc. Soc. Photo-Opt. Instrum. Eng. (SPIE) CS-10703, 107035P. DOI. ADS.

Johnson, L.C., Upton, R., Rimmele, T., Barden, S.: 2012, Quasi-static wavefront control for the Advanced Technology Solar Telescope. In: Stepp, L.M., Gilmozzi, R., Hall, H.J. (eds.) Ground-Based and Airborne Telescopes IV, Proc. Soc. Photo-Opt. Instrum. Eng. (SPIE) CS-8444, 84443O. DOI. ADS.

Johnson, L.C., Cummings, K., Drobilek, M., Gregory, S., Hegwer, S., Johansson, E., Marino, J., Richards, K., Rimmele, T., Sekulic, P., Wöger, F.: 2014, Solar adaptive optics with the DKIST: Status report. In: Marchetti, E., Close, L.M., Véran, J.-P. (eds.) Adaptive Optics Systems IV, Proc. Soc. Photo-Opt. Instrum. Eng. (SPIE) CS-9148, 91481S. DOI. ADS.

Johnson, L.C., Cummings, K., Drobilek, M., Johansson, E., Marino, J., Richards, K., Rimmele, T., Sekulic, P., Wöger, F.: 2016, Status of the DKIST system for solar adaptive optics. In: Marchetti, E., Close, L.M., Véran, J.-P. (eds.) Adaptive Optics Systems V, Proc. Soc. Photo-Opt. Instrum. Eng. (SPIE) CS-9909, 99090Y. DOI. ADS.

Johnson, L.C., Cummings, K., Drobilek, M., Johansson, E., Marino, J., Rampy, R., Richards, K., Rimmele, T., Sekulic, P., Wöger, F.: 2018, Laboratory integration of the DKIST wavefront correction system. In: Close, L.M., Schreiber, L., Schmidt, D. (eds.) Adaptive Optics Systems VI, Proc. Soc. Photo-Opt. Instrum. Eng. (SPIE) CS-10703, 107030F. DOI. ADS.

Johnson, L.C., Johansson, E., Richards, K., Wöger, F., Marino, J., Rimmele, T.: 2020, First light with adaptive optics: The performance of the DKIST high-order adaptive optics. In: Proc. Soc. Photo-Opt. Instrum. Eng. (SPIE), submitted. 
Judge, P.G., Tomczyk, S., Livingston, W.C., Keller, C.U., Penn, M.J.: 2002, Spectroscopic detection of the 3.934 micron line of Si IX in the solar corona. Astrophys. J. Lett. 576, L157. DOI. ADS.

Kärcher, H.J., Weis, U., Dreyer, O., Jeffers, P.F., Bonomi, G.: 2012, The azimuth axes mechanisms for the ATST telescope mount assembly. In: Stepp, L.M., Gilmozzi, R., Hall, H.J. (eds.) Ground-Based and Airborne Telescopes IV, Proc. Soc. Photo-Opt. Instrum. Eng. (SPIE) CS-8444, 84440A. DOI. ADS.

Keil, S., Rimmele, T., Keller, C. (ATST Team): 2003, Design and development of the Advanced Technology Solar Telescope. Astron. Nachr. 324, 303. DOI. ADS.

Keil, S.L., Rimmele, T.R., Wagner, J. (ATST Team): 2010, Advanced Technology Solar Telescope: A status report. Astron. Nachr. 331, 609. DOI. ADS.

Keil, S.L., Rimmele, T., Keller, C.U., Hill, F., Radick, R.R., Oschmann, J.M., Warner, M., Dalrymple, N.E., Briggs, J., Hegwer, S.L., Ren, D.: 2003, Design and development of the Advanced Technology Solar Telescope (ATST). In: Keil, S.L., Avakyan, S.V. (eds.) Innovative Telescopes and Instrumentation for Solar Astrophysics, Proc. Soc. Photo-Opt. Instrum. Eng. (SPIE) CS-4853, 240. DOI. ADS.

Keil, S.L., Rimmele, T.R., Oschmann, J., Hubbard, R., Warner, M., Price, R., Dalrymple, N. (ATST Team): 2004, Science goals and development of the advanced technology solar telescope. In: Stepanov, A.V., Benevolenskaya, E.E., Kosovichev, A.G. (eds.) Multi-Wavelength Investigations of Solar Activity, IAU Symp. 223, 581. DOI. ADS.

Kentischer, T.J., Schmidt, W., von der Lühe, O., Sigwarth, M., Bell, A., Halbgewachs, C., Fischer, A.: 2012, The visible tunable filtergraph for the ATST. In: McLean, I.S., Ramsay, S.K., Takami, H. (eds.) GroundBased and Airborne Instrumentation for Astronomy IV, Proc. Soc. Photo-Opt. Instrum. Eng. (SPIE) CS-8446, 844677. DOI. ADS.

Kuhn, J.R., Penn, M.J., Mann, I.: 1996, The near-infrared coronal spectrum. Astrophys. J. Lett. 456, L67. DOI. ADS.

Kuhn, J.R., MacQueen, R.M., Streete, J., Tansey, G., Mann, I., Hillebrand, P., Coulter, R., Lin, H., Edmunds, D., Judge, P.: 1999, Probable detection of a bright infrared coronal emission line of Si IX near 3.93 microns. Astrophys. J. 521, 478. DOI. ADS.

Kuhn, J.R., Coulter, R., Lin, H., Mickey, D.L.: 2003, The SOLARC off-axis coronagraph. In: Keil, S.L., Avakyan, S.V. (eds.) Innovative Telescopes and Instrumentation for Solar Astrophysics, Proc. Soc. Photo-Opt. Instrum. Eng. (SPIE) CS-4853, 318. DOI. ADS.

Kuhn, J.R., Bond, T., Mickey, D., Scholl, I.: 2013, The ATST coronal cryogenic infrared spectropolarimeter. In: Solar Heliospheric and INterplanetary Environment (SHINE 2013), 47. ADS.

Langhans, K., Scharmer, G.B., Kiselman, D., Löfdahl, M.G.: 2007, Observations of dark-cored filaments in sunspot penumbrae. Astron. Astrophys. 464, 763. DOI. ADS.

Lin, H., Kuhn, J.R., Coulter, R.: 2004, Coronal magnetic field measurements. Astrophys. J. Lett. 613, L177. DOI. ADS.

Lin, H., Penn, M.J.: 2004, The Advanced Technology Solar Telescope site survey sky brightness monitor. Publ. Astron. Soc. Pac. 116, 652. DOI. ADS.

Lin, H., Penn, M.J., Tomczyk, S.: 2000, A new precise measurement of the coronal magnetic field strength. Astrophys. J. Lett. 541, L83. DOI. ADS.

Lin, H., Rimmele, T.: 1999, The granular magnetic fields of the quiet Sun. Astrophys. J. 514, 448. DOI. ADS.

Lites, B.W.: 2002, Characterization of magnetic flux in the quiet Sun. Astrophys. J. 573, 431. DOI. ADS.

Lites, B.W.: 2009, A new perspective on quiet Sun magnetism. Sci. China Ser. G, Phys. Mech. Astron. 52, 1660. DOI. ADS.

Lites, B.W., Kubo, M., Socas-Navarro, H., Berger, T., Frank, Z., Shine, R., Tarbell, T., Title, A., Ichimoto, K., Katsukawa, Y., Tsuneta, S., Suematsu, Y., Shimizu, T., Nagata, S.: 2008, The horizontal magnetic flux of the quiet-Sun internetwork as observed with the hinode spectro-polarimeter. Astrophys. J. 672, 1237. DOI. ADS.

Lites, B.W., Rempel, M., Borrero, J.M., Danilovic, S.: 2017, Are internetwork magnetic fields in the solar photosphere horizontal or vertical? Astrophys. J. 835, 14. DOI. ADS.

Martínez Pillet, V., Tritschler, A., Harra, L., Andretta, V., Vourlidas, A., Raouafi, N., Alterman, B.L., Bellot Rubio, L., Cauzzi, G., Cranmer, S.R., Gibson, S., Habbal, S., Ko, Y.K., Lepri, S.T., Linker, J., Malaspina, D.M., Matthews, S., Parenti, S., Petrie, G., Spadaro, D., Ugarte-Urra, I., Warren, H., Winslow, R.: 2020, Solar physics in the 2020s: DKIST, parker solar probe, and solar orbiter as a multi-messenger constellation. ADS. arXiv.

McMullin, J.P., Rimmele, T.R., Keil, S.L., Warner, M., Barden, S., Bulau, S., Craig, S., Goodrich, B., Hansen, E., Hegwer, S., Hubbard, R., McBride, W., Shimko, S., Wöger, F., Ditsler, J.: 2012, The Advanced Technology Solar Telescope: Design and early construction. In: Stepp, L.M., Gilmozzi, R., Hall, H.J. (eds.) Ground-Based and Airborne Telescopes IV, Proc. Soc. Photo-Opt. Instrum. Eng. (SPIE) CS-8444, 844407. DOI. ADS.

McMullin, J.P., Rimmele, T.R., Martínez Pillet, V., Berger, T.E., Casini, R., Craig, S.C., Elmore, D.F., Goodrich, B.D., Hegwer, S.L., Hubbard, R.P., Johansson, E.M., Kuhn, J.R., Lin, H., McVeigh, W., 
Schmidt, W., Shimko, S., Tritschler, A., Warner, M., Wöger, F.: 2014, Construction status of the Daniel K. Inouye Solar Telescope. In: Stepp, L.M., Gilmozzi, R., Hall, H.J. (eds.) Ground-Based and Airborne Telescopes V, Proc. Soc. Photo-Opt. Instrum. Eng. (SPIE) CS-9145, 914525. DOI. ADS.

McMullin, J.P., Rimmele, T.R., Warner, M., Martínez Pillet, V., Casini, R., Berukoff, S., Craig, S.C., Elmore, D., Ferayorni, A., Goodrich, B.D., Hubbard, R.P., Harrington, D., Hegwer, S., Jeffers, P., Johansson, E.M., Kuhn, J., Lin, H., Marshall, H., Mathioudakis, M., McBride, W.R., McVeigh, W., Phelps, L., Schmidt, W., Shimko, S., Sueoka, S., Tritschler, A., Williams, T.R., Wöger, F.: 2016, Construction status of the Daniel K. Inouye solar telescope. In: Hall, H.J., Gilmozzi, R., Marshall, H.K. (eds.) Ground-Based and Airborne Telescopes VI, Proc. Soc. Photo-Opt. Instrum. Eng. (SPIE) CS-9906, 99061B. DOI. ADS.

McQuillen, I., Phelps, L.: 2018, Factory acceptance testing and model refinement for the Daniel K. Inouye Solar Telescope air knife assembly. In: Angeli, G.Z., Dierickx, P. (eds.) Modeling, Systems Engineering, and Project Management for Astronomy VIII, Proc. Soc. Photo-Opt. Instrum. Eng. (SPIE) CS-10705, 1070525. DOI. ADS.

McQuillen, I., Phelps, L., Warner, M., Hubbard, R.: 2016, Daniel K. Inouye Solar Telescope: Computational fluid dynamic analyses and evaluation of the air knife model. In: Angeli, G.Z., Dierickx, P. (eds.) Modeling, Systems Engineering, and Project Management for Astronomy VII, Proc. Soc. Photo-Opt. Instrum. Eng. (SPIE) CS-9911, 99111M. DOI. ADS.

Moran, T., Deming, D., Jennings, D.E., McCabe, G.: 2000, Solar magnetic field studies using the 12 micron emission lines. III. Simultaneous measurements at 12 and 1.6 microns. Astrophys. J. 533, 1035. DOI. ADS.

Murga, G., Marshall, H., Phelps, L., Hervás, A., Larracoechea, I.: 2011, ATST enclosure mechanical and thermal models. In: Andersen, T., Enmark, A. (eds.) Integrated Modeling of Complex Optomechanical Systems, Proc. Soc. Photo-Opt. Instrum. Eng. (SPIE) CS-8336, 83360K. DOI. ADS.

Murga, G., Marshall, H., Ariño, J., Lorentz, T.: 2012, ATST enclosure final design and construction plans. In: Stepp, L.M., Gilmozzi, R., Hall, H.J. (eds.) Ground-Based and Airborne Telescopes IV, Proc. Soc. Photo-Opt. Instrum. Eng. (SPIE) CS-8444, 844408. DOI. ADS.

Murga, G., Marshall, H.K., Lorentz, T.E., Ariño, J., Ampuero, P.: 2014, DKIST enclosure fabrication factory assembly and testing. In: Stepp, L.M., Gilmozzi, R., Hall, H.J. (eds.) Ground-Based and Airborne Telescopes V, Proc. Soc. Photo-Opt. Instrum. Eng. (SPIE) CS-9145, 914527. DOI. ADS.

National Research Council Astronomy and Astrophysics Survey Committee: 2000, Astronomy and Astrophysics in the New Millennium, NAS, Washington, DC. ADS.

Nelson, P.G., Casini, R., de Wijn, A.G., Knoelker, M.: 2010, The Visible Spectro-Polarimeter (ViSP) for the Advanced Technology Solar Telescope. In: McLean, I.S., Ramsay, S.K., Takami, H. (eds.) GroundBased and Airborne Instrumentation for Astronomy III, Proc. Soc. Photo-Opt. Instrum. Eng. (SPIE) CS-7735, 77358C. DOI. ADS.

Oh, C.J., Lowman, A.E., Smith, G.A., Su, P., Huang, R., Su, T., Kim, D., Zhao, C., Zhou, P., Burge, J.H.: 2016, Fabrication and testing of $4.2 \mathrm{~m}$ off-axis aspheric primary mirror of Daniel K. Inouye Solar Telescope. In: Navarro, R., Burge, J.H. (eds.) Advances in Optical and Mechanical Technologies for Telescopes and Instrumentation II, Proc. Soc. Photo-Opt. Instrum. Eng. (SPIE) CS-9912, 99120O. DOI. ADS.

Oschmann, J.M. Jr.: 2004, Systems engineering in ground-based astronomy. In: Craig, S.C., Cullum, M.J. (eds.) Modeling and Systems Engineering for Astronomy, Proc. Soc. Photo-Opt. Instrum. Eng. (SPIE) CS-5497, 1. DOI. ADS.

Oschmann, J., Dalrymple, N., Warner, M., Price, R., Hill, F., Hubbard, R., Rimmele, T.R., Keller, C.U., Keil, S.: 2004, Advanced Technology Solar Telescope: A progress report. In: Fineschi, S., Gummin, M.A. (eds.) Telescopes and Instrumentation for Solar Astrophysics, Proc. Soc. Photo-Opt. Instrum. Eng. (SPIE) CS-5171, 160. DOI. ADS.

Parker, E.N.: 2000, The physics of the Sun and the gateway to the stars. Phys. Today 53, 26. DOI. ADS.

Penn, M.J., Kuhn, J.R.: 1994, Ground-based detection of an infrared [Si X] coronal emission line and improved wavelengths for the infrared [Fe XIII] emission lines. Astrophys. J. 434, 807. DOI. ADS.

Phelps, L., Warner, M.: 2008, Advanced Technology Solar Telescope lower enclosure thermal system. In: Angeli, G.Z., Cullum, M.J. (eds.) Modeling, Systems Engineering, and Project Management for Astronomy III, Proc. Soc. Photo-Opt. Instrum. Eng. (SPIE) CS-7017, 701719. DOI. ADS.

Phelps, L., Rimmele, T., Hubbard, R.P., Elmore, D.: 2010, The Advanced Technology Solar Telescope Coudé lab thermal environment. In: Stepp, L.M., Gilmozzi, R., Hall, H.J. (eds.) Ground-Based and Airborne Telescopes III, Proc. Soc. Photo-Opt. Instrum. Eng. (SPIE) CS-7733, 77333U. DOI. ADS.

Phelps, L., Murga, G., Fraser, M., Climent, T.: 2012, Facility level thermal systems for the Advanced Technology Solar Telescope. In: Stepp, L.M., Gilmozzi, R., Hall, H.J. (eds.) Ground-Based and Airborne Telescopes IV, Proc. Soc. Photo-Opt. Instrum. Eng. (SPIE) CS-8444, 84443M. DOI. ADS.

Phelps, L., Murga, G., Montijo, G., Hauth, D.: 2014, Plate coil thermal test bench for the Daniel K. Inouye Solar Telescope (DKIST) carousel cooling system. In: Angeli, G.Z., Dierickx, P. (eds.) Modeling, Sys- 
tems Engineering, and Project Management for Astronomy VI, Proc. Soc. Photo-Opt. Instrum. Eng. (SPIE) CS-9150, 91501Q. DOI. ADS.

Pietarila Graham, J., Danilovic, S., Schüssler, M.: 2009, Turbulent magnetic fields in the quiet Sun: Implications of hinode observations and small-scale dynamo simulations. Astrophys. J. 693, 1728. DOI. ADS.

Rast, M., Cauzzi, G., Martínez Pillet, V.: 2019, The critical science plan for DKIST. Nuovo Cim. C Geophys. Space Phys. C 42, 7. DOI. ADS.

Rast, M., Bello Gonzalez, N., Bellot Rubio, L., Cao, W., Cauzzi, G., DeLuca, E., De Pontieu, B., Fletcher, L., Gibson, S., Judge, P., Katsukawa, Y., Kazachenko, M., Khomenko, E., Landi, E., Martínez Pillet, V., Petrie, G., Qiu, J., Rachmeler, L., Rempel, M., Schmidt, W., Scullion, E., Sun, X., Welsch, B., Andretta, V., Ayres, T., Ballai, I., Balasubramaniam, K.S., Berger, T., Bradshaw, S., Carlsson, M., Casini, R., Centeno, R., Cranmer, S., DeForest, C., Deng, Y., Fedun, V., Fischer, C., Gonzalez Manrique, S., Hahn, M., Harra, L., Henriques, V., Hurlburt, N., Jaeggli, S., Jain, R., Jeffries, S., Kowalski, A., Kuckein, C., Kuhn, J., Liu, J., Liu, W., Longcope, D., McAteer, J., McIntosh, S., McKenzie, D., Morton, R., Muglach, K., Miralles, M.P., Parnell, C., Poduval, B., Reep, J., Suematsu, Y., Schad, T., Schmit, D., Socas-Navarro, H., Srivastava, A., Tarr, L., Tiwari, S., Tritschler, A., Verth, G., Vourlidas, A., Wang, H., Wang, Y.M.: 2021, The critical science plan for DKIST. Solar Phys. 296, submitted.

Rempel, M.: 2012, Numerical sunspot models: Robustness of photospheric velocity and magnetic field structure. Astrophys. J. 750, 62. DOI. ADS.

Rempel, M.: 2014, Numerical simulations of quiet Sun magnetism: On the contribution from a small-scale dynamo. Astrophys. J. 789, 132. DOI. ADS. arXiv.

Rempel, M.: 2017, Extension of the MURaM radiative MHD code for coronal simulations. Astrophys. J. 834, 10. DOI. ADS.

Rempel, M.: 2018, Small-scale dynamo simulations: Magnetic field amplification in exploding granules and the role of deep and shallow recirculation. Astrophys. J. 859, 161. DOI. ADS.

Rempel, M.: 2020, On the contribution of quiet-Sun magnetism to solar irradiance variations: Constraints on quiet-Sun variability and grand-minimum scenarios. Astrophys. J. 894, 140. DOI. ADS.

Rempel, M., Schlichenmaier, R.: 2011, Sunspot modeling: From simplified models to radiative MHD simulations. Liv. Rev. Solar Phys. 8, 3. DOI. ADS.

Richards, K., Rimmele, T., Hegwer, S.L., Upton, R.S., Woeger, F., Marino, J., Gregory, S., Goodrich, B.: 2010, The adaptive optics and wavefront correction systems for the Advanced Technology Solar Telescope. In: Ellerbroek, B.L., Hart, M., Hubin, N., Wizinowich, P.L. (eds.) Adaptive Optics Systems II, Proc. Soc. Photo-Opt. Instrum. Eng. (SPIE) CS-7736, 773608. DOI. ADS.

Rimmele, T.R.: 2000, Solar adaptive optics. In: Wizinowich, P.L. (ed.) Adaptive Optical Systems Technology, Proc. Soc. Photo-Opt. Instrum. Eng. (SPIE) CS-4007, 218. DOI. ADS.

Rimmele, T., the ATST Science Working Group: 2005, Science Requirements Document, DKIST Project Documentation SPEC-0001. nso.edu/wp-content/uploads/2020/10/SPEC-0001_SRD_RevB.pdf.

Rimmele, T.R., ATST Team: 2008, The unique scientific capabilities of the Advanced Technology Solar Telescope. Adv. Space Res. 42, 78. DOI. ADS.

Rimmele, T.R., Marino, J.: 2011, Solar adaptive optics. Living Rev. Solar Phys. 8, 2. DOI. ADS.

Rimmele, T.R., Radick, R.R.: 1998, Solar adaptive optics at the National Solar Observatory. In: Bonaccini, D., Tyson, R.K. (eds.) Adaptive Optical System Technologies, Proc. Soc. Photo-Opt. Instrum. Eng. (SPIE) CS-3353, 72. DOI. ADS.

Rimmele, T., Keil, S., Wagner, J. (ATST Team): 2008, Status of the Advanced Technology Solar Telescope. In: Euro. Solar Phys. Meet. 12, 6.2. ADS.

Rimmele, T., Keller, C., Keil, S., Hill, F. (ATST Team): 2001, The Advanced Technology Solar Telescope: Science goals and instrument description. In: Astron. Gesell. Meeting Abs 18, MS 10 06. ADS.

Rimmele, T.R., Keil, S.L., Keller, C.U., Hill, F., Briggs, J., Dalrymple, N.E., Goodrich, B.D., Hegwer, S.L., Hubbard, R., Oschmann, J.M., Radick, R.R., Ren, D., Wagner, J., Wampler, S., Warner, M.: 2003a, Technical challenges of the Advanced Technology Solar Telescope. In: Oschmann, J.M., Stepp, L.M. (eds.) Large Ground-Based Telescopes, Proc. Soc. Photo-Opt. Instrum. Eng. (SPIE) CS-4837, 94. DOI. ADS.

Rimmele, T., Keil, S.L., Keller, C., Hill, F., Penn, M., Goodrich, B., Hegwer, S., Hubbard, R., Oschmann, J., Warner, M., Dalrymple, N., Radick, R. (ATST Team): 2003b, Science objectives and technical challenges of the advanced technology solar telescope (invited review). In: Pevtsov, A.A., Uitenbroek, H. (eds.) Current Theoretical Models and Future High Resolution Solar Observations: Preparing for ATST CS-286, Astron. Soc. Pacific, San Francisco, 3. ADS.

Rimmele, T.R., Hubbard, R.P., Balasubramaniam, K.S., Berger, T., Elmore, D., Gary, G.A., Jennings, D., Keller, C., Kuhn, J., Lin, H., Mickey, D., Moretto, G., Socas-Navarro, H., Stenflo, J.O., Wang, H.: 2004, Instrumentation for the Advanced Technology Solar Telescope. In: Moorwood, A.F.M., Iye, M. (eds.) Ground-Based Instrumentation for Astronomy, Proc. Soc. Photo-Opt. Instrum. Eng. (SPIE) CS-5492, 944. DOI. ADS. 
Rimmele, T.R., Keil, S., Wagner, J., Dalrymple, N., Goodrich, B., Hansen, E., Hill, F., Hubbard, R., Phelps, L., Richards, K., Warner, M.: 2005, Advanced Technology Solar Telescope: A progress report. In: Fineschi, S., Viereck, R.A. (eds.) Solar Physics and Space Weather Instrumentation, Proc. Soc. Photo-Opt. Instrum. Eng. (SPIE) CS-5901, 41. DOI. ADS.

Rimmele, T., Richards, K., Roche, J.M., Hegwer, S.L., Hubbard, R.P., Hansen, E.R., Goodrich, B., Upton, R.S.: 2006, The wavefront correction system for the Advanced Technology Solar Telescope. In: Ellerbroek, B.L., Calia, D.B. (eds.) Advances in Adaptive Optics II, Proc. Soc. Photo-Opt. Instrum. Eng. (SPIE) CS-6272, 627212. DOI. ADS

Rimmele, T.R., Wagner, J., Keil, S., Elmore, D., Hubbard, R., Hansen, E., Warner, M., Jeffers, P., Phelps, L., Marshall, H., Goodrich, B., Richards, K., Hegwer, S., Kneale, R., Ditsler, J.: 2010, The Advanced Technology Solar Telescope: Beginning construction of the world's largest solar telescope. In: Stepp, L.M., Gilmozzi, R., Hall, H.J. (eds.) Ground-Based and Airborne Telescopes III, Proc. Soc. Photo-Opt. Instrum. Eng. (SPIE) CS-7733, 77330G. DOI. ADS.

Rimmele, T.R., Keil, S., McMullin, J., Knölker, M., Kuhn, J.R., Goode, P.R., Rosner, R., Casini, R., Lin, H., Tritschler, A., Wöger, F. (ATST Team): 2012, Construction of the Advanced Technology Solar Telescope. In: Rimmele, T.R., Tritschler, A., Wöger, F., Collados Vera, M., Socas-Navarro, H., Schlichenmaier, R., Carlsson, M., Berger, T., Cadavid, A., Gilbert, P.R., Goode, P.R., Knölker, M. (eds.) Second ATST-EAST Meeting: Magnetic Fields from the Photosphere to the Corona CS-463, Astron. Soc. Pacific, San Francisco, 377. ADS.

Rimmele, T., Berger, T., McMullin, J., Warner, M., Casinsi, R., Kuhn, J., Lin, H., Woeger, F., Schmidt, W., Tritschler, A., Inouye, D.K., (Solar Telescope Team): 2014, The Daniel K. Inouye Solar Telescope: A project update. In: Ryan, S. (ed.) Adv. Maui Optical and Space Surv. Technologies Conf., The Maui Economic Development Board, Wailea, Maui, Hawai'i, E43. ADS.

Schlichenmaier, R., Bellot Rubio, L.R., Collados, M., Erdelyi, R., Feller, A., Fletcher, L., Jurcak, J., Khomenko, E., Leenaarts, J., Matthews, S., Belluzzi, L., Carlsson, M., Dalmasse, K., Danilovic, S., Gömöry, P., Kuckein, C., Manso Sainz, R., Martinez Gonzalez, M., Mathioudakis, M., Ortiz, A., Riethmüller, T.L., Rouppe van der Voort, L., Simoes, P.J.A., Trujillo Bueno, J., Utz, D., Zuccarello, F.: 2019, Science Requirement Document (SRD) for the European Solar Telescope (EST) (2nd edition, December 2019). ADS. arXiv.

Schmidt, W., von der Lühe, O., Volkmer, R., Denker, C., Solanki, S.K., Balthasar, H., Bello González, N., Berkefeld, T., Collados Vera, M., Hofmann, A., Kneer, F., Lagg, A., Puschmann, K.G., Schmidt, D., Sobotka, M., Soltau, D., Strassmeier, K.G.: 2012, The GREGOR Solar Telescope on Tenerife. In: Rimmele, T.R., Tritschler, A., Wöger, F., Collados Vera, M., Socas-Navarro, H., Schlichenmaier, R., Carlsson, M., Berger, T., Cadavid, A., Gilbert, P.R., Goode, P.R., Knölker, M. (eds.) Second ATST-EAST Meeting: Magnetic Fields from the Photosphere to the Corona CS-463, Astron. Soc. Pacific, San Francisco, 365. ADS. arXiv.

Schmidt, W., Bell, A., Halbgewachs, C., Heidecke, F., Kentischer, T.J., von der Lühe, O., Scheiffelen, T., Sigwarth, M.: 2014, A two-dimensional spectropolarimeter as a first-light instrument for the Daniel K. Inouye Solar Telescope. In: Ramsay, S.K., McLean, I.S., Takami, H. (eds.) Ground-Based and Airborne Instrumentation for Astronomy V, Proc. Soc. Photo-Opt. Instrum. Eng. (SPIE) CS-9147, 91470E. DOI. ADS.

Schmidt, W., Schubert, M., Ellwarth, M., Baumgartner, J., Bell, A., Fischer, A., Halbgewachs, C., Heidecke, F., Kentischer, T., von der Lühe, O., Scheiffelen, T., Sigwarth, M.: 2016, End-to-end simulations of the visible tunable filter for the Daniel K. Inouye Solar Telescope. In: Evans, C.J., Simard, L., Takami, H. (eds.) Ground-Based and Airborne Instrumentation for Astronomy VI, Proc. Soc. Photo-Opt. Instrum. Eng. (SPIE) CS-9908, 99084N. DOI. ADS. arXiv.

Schmidt, D., Gorceix, N., Goode, P.R., Marino, J., Rimmele, T., Berkefeld, T., Wöger, F., Zhang, X., Rigaut, F., von der Lühe, O.: 2017, Clear widens the field for observations of the Sun with multi-conjugate adaptive optics. Astron. Astrophys. 597, L8. DOI. ADS.

Schmidt, D., Marino, J., Gorceix, N., Rimmele, T., Johnson, L., Berkefeld, T., Goode, P.: 2018, From clear to DKIST: Advancing solar MCAO from 1.6 to 4 meters. In: Close, L.M., Schreiber, L., Schmidt, D. (eds.) Adaptive Optics Systems VI, Proc. Soc. Photo-Opt. Instrum. Eng. (SPIE) CS-10703, 1070326. DOI. ADS.

Schüssler, M.: 2005, Flux tubes, surface magnetism, and the solar dynamo: Constraints and open problems. Astron. Nachr. 326, 194. DOI. ADS.

Schüssler, M., Vögler, A.: 2006, Magnetoconvection in a sunspot umbra. Astrophys. J. Lett. 641, L73. DOI. ADS.

Sekulic, P., Liang, C., Gonzales, K., Hubbard, R.P., Craig, S.C.: 2016, Daniel K. Inouye Solar Telescope optical alignment plan. In: Hall, H.J., Gilmozzi, R., Marshall, H.K. (eds.) Ground-Based and Airborne Telescopes VI, Proc. Soc. Photo-Opt. Instrum. Eng. (SPIE) CS-9906, 990653. DOI. ADS. 
Sekulic, P., Jeffers, P.F., Puentes, M., Smith, B., Liang, M., De Vanssay, J.-B., Liang, C., Haar, S.: 2020, Lessons learned of the Daniel K. Inouye Solar Telescope optical alignment. In: Proc. Soc. Photo-Opt. Instrum. Eng. (SPIE), submitted.

Soida, M., Otmianowska-Mazur, K., de Gouveia Dal Pino, E.M., Lazarian, A. (eds.): 2012, Magnetic Fields in the Universe III - From Laboratory and Stars to Primordial Structures, Jagiellonian University, Astronomical Observatory, Zakopane, Poland. ADS.

Stein, R.F.: 2012, Solar surface magneto-convection. Liv. Rev. Solar Phys. 9, 4. DOI. ADS.

Stein, R.F., Nordlund, Å.: 2006, Solar small-scale magnetoconvection. Astrophys. J. 642, 1246. DOI. ADS.

Stein, R.F., Nordlund, A.: 2012, Flux emergence and pore formation: What ATST can see. In: Rimmele, T.R., Tritschler, A., Wöger, F., Collados Vera, M., Socas-Navarro, H., Schlichenmaier, R., Carlsson, M., Berger, T., Cadavid, A., Gilbert, P.R., Goode, P.R., Knölker, M. (eds.) Second ATST-EAST Meeting: Magnetic Fields from the Photosphere to the Corona CS-463, Astron. Soc. Pacific, San Francisco, 83. ADS.

Stenflo, J.: 1994, Solar Magnetic Fields: Polarized Radiation Diagnostics, Astrophys. Space Sci. Lib. 189, Springer, Berlin. DOI. ADS.

Stenflo, J.: 2000, Solar photosphere: Intranetwork and turbulent magnetic fields. In: Murdin, P. (ed.) Encyclopedia of Astronomy and Astrophysics, 2009. ADS. eaa.crcpress.com/articles/pdf/2009.pdf.

Stenflo, J.O.: 2008, Solar magnetic fields. J. Astrophys. Astron. 29, 19. DOI. ADS.

Stenflo, J.O.: 2013, Solar magnetic fields as revealed by Stokes polarimetry. Astron. Astrophys. Rev. 21 , 66. DOI. ADS.

Stenflo, J.O., Keller, C.U., Gandorfer, A.: 1998, Differential Hanle effect and the spatial variation of turbulent magnetic fields on the Sun. Astron. Astrophys. 329, 319 ADS.

Tayabaly, K.: 2013, Prediction of the BRDF with microfinish topographer roughness measurements. Master's thesis, University of Arizona.

Trischler, A.: 2020, Operational Concepts Document, DKIST Project Documentation SPEC-0036. nso.edu/wp-content/uploads/2020/10/SPEC-0036_OCD_RevD.pdf.

Tritschler, A., Rimmele, T.R., Berukoff, S., Casini, R., Kuhn, J.R., Lin, H., Rast, M.P., McMullin, J.P., Schmidt, W., Wöger, F. (DKIST Team): 2016, Daniel K. Inouye Solar Telescope: High-resolution observing of the dynamic Sun. Astron. Nachr. 337, 1064. DOI. ADS.

Tritschler, A., et al.: 2021, The DKIST operations. Solar Phys. 296, in preparation.

Tsuneta, S., Ichimoto, K., Katsukawa, Y., Nagata, S., Otsubo, M., Shimizu, T., Suematsu, Y., Nakagiri, M., Noguchi, M., Tarbell, T., Title, A., Shine, R., Rosenberg, W., Hoffmann, C., Jurcevich, B., Kushner, G., Levay, M., Lites, B., Elmore, D., Matsushita, T., Kawaguchi, N., Saito, H., Mikami, I., Hill, L.D., Owens, J.K.: 2008, The Solar Optical Telescope for the hinode mission: An overview. Solar Phys. 249, 167. DOI. ADS.

Vögler, A., Shelyag, S., Schüssler, M., Cattaneo, F., Emonet, T., Linde, T.: 2005, Simulations of magnetoconvection in the solar photosphere. Equations, methods, and results of the MURaM code. Astron. Astrophys. 429, 335. DOI. ADS.

von der Lühe, O., et al.: 2021, The DKIST visible tunable filter. Solar Phys. 296, in preparation.

Wagner, J., Rimmele, T.R., Keil, S., Barr, J., Dalrymple, N., Ditsler, J., Goodrich, B., Hansen, E., Hegwer, S., Hill, F., Hubbard, R., Phelps, L., Price, R., Richards, K., Warner, M.: 2006, Advanced Technology Solar Telescope: A progress report. In: Stepp, L.M. (ed.) Ground-Based and Airborne Telescopes, Proc. Soc. Photo-Opt. Instrum. Eng. (SPIE) CS-6267, 626709. DOI. ADS.

Wampler, S., Goodrich, B.: 2004, The ATST virtual instrument model. In: Ochsenbein, F., Allen, M.G., Egret, D. (eds.) Astronomical Data Analysis Software and Systems (ADASS) XIII CS-314, Astron. Soc. Pacific, San Francisco, 820. ADS.

Wampler, S., Goodrich, B.: 2009, A scalable data handling system for ATST. In: Bohlender, D.A., Durand, D., Dowler, P. (eds.) Astronomical Data Analysis Software and Systems XVIII CS-411, Astron. Soc. Pacific, San Francisco, 527. ADS.

Warner, M., Cho, M., Goodrich, B., Hansen, E., Hubbard, R., Lee, J.P., Wagner, J.: 2006, The Advanced Technology Solar Telescope mount assembly. In: Stepp, L.M. (ed.) Ground-Based and Airborne Telescopes, Proc. Soc. Photo-Opt. Instrum. Eng. (SPIE) CS-6267, 62673C. DOI. ADS.

Warner, M., McMullin, J., Rimmele, T., Berger, T.: 2013, The Advanced Technology Solar Telescope (ATST) project: A construction update. In: Fineschi, S., Fennelly, J. (eds.) Solar Physics and Space Weather Instrumentation V, Proc. Soc. Photo-Opt. Instrum. Eng. (SPIE) CS-8862, 88620D. DOI. ADS.

Warner, M., Rimmele, T.R., Martínez Pillet, V., Casini, R., Berukoff, S., Craig, S.C., Ferayorni, A., Goodrich, B.D., Hubbard, R.P., Harrington, D., Jeffers, P., Johansson, E.M., Kneale, R., Kuhn, J., Liang, C., Lin, H., Marshall, H., Mathioudakis, M., McBride, W.R., McMullin, J., McVeigh, W., Sekulic, P., Schmidt, W., Shimko, S., Sueoka, S., Summers, R., Tritschler, A., Williams, T.R., Wöger, F.: 2018, Construction update of the Daniel K. Inouye Solar Telescope project. In: Marshall, H.K., Spyromilio, J. (eds.) Ground- 
Based and Airborne Telescopes VII, Proc. Soc. Photo-Opt. Instrum. Eng. (SPIE) CS-10700, 107000V. DOI. ADS.

Warner, M., Rimmele, T.R., Martínez Pillet, V., Casini, R., Berukoff, S., Craig, S.C., Ferayorni, A., Goodrich, B.D., Hubbard, R.P., Harrington, D., Jeffers, P., Johansson, E.M., Kneale, R., Kuhn, J., Liang, C., Lin, H., Marshall, H., Mathioudakis, M., McBride, W.R., McMullin, J., McVeigh, W., Sekulic, P., Schmidt, W., Shimko, S., Sueoka, S., Summers, R., Tritschler, A., Williams, T.R., Wöger, F.: 2020, Construction update of the Daniel K. Inouye Solar Telescope project. In: Ground-Based and Airborne Telescopes VII, Proc. Soc. Photo-Opt. Instrum. Eng. (SPIE) CS-10700, 107000V. DOI. ADS.

Wedemeyer-Böhm, S., Lagg, A., Nordlund, Å.: 2009, Coupling from the photosphere to the chromosphere and the corona. Space Sci. Rev. 144, 317. DOI. ADS.

White, C.R., Phelps, L.: 2016, DKIST facility management system integration. In: Hall, H.J., Gilmozzi, R., Marshall, H.K. (eds.) Ground-Based and Airborne Telescopes VI, Proc. Soc. Photo-Opt. Instrum. Eng. (SPIE) CS-9906, 990652. DOI. ADS.

Whittet, D.C.B., Martin, P.G., Hough, J.H., Rouse, M.F., Bailey, J.A., Axon, D.J.: 1992, Systematic variations in the wavelength dependence of interstellar linear polarization. Astrophys. J. 386, 562. DOI. ADS.

Wiegelmann, T., Thalmann, J.K., Solanki, S.K.: 2014, The magnetic field in the solar atmosphere. Astron. Astrophys. Rev. 22, 78. DOI. ADS.

Wöger, F.: 2010, Optical transfer functions derived from solar adaptive optics system data. Appl. Opt. 49, 1818.

Wöger, F.: 2014, DKIST visible broadband imager interference filters. In: Ramsay, S.K., McLean, I.S., Takami, H. (eds.) Ground-Based and Airborne Instrumentation for Astronomy V, Proc. Soc. Photo-Opt. Instrum. Eng. (SPIE) CS-9147, 91479I. DOI. ADS.

Wöger, F., Uitenbroek, H., Tritschler, A.r., McBride, W., Elmore, D., Rimmele, T., Cowan, B., Wampler, S., Goodrich, B.: 2010, The ATST visible broadband imager: A case study for real-time image reconstruction and optimal data handling. In: McLean, I.S., Ramsay, S.K., Takami, H. (eds.) Ground-Based and Airborne Instrumentation for Astronomy III, Proc. Soc. Photo-Opt. Instrum. Eng. (SPIE) CS-7735, 773521. DOI. ADS.

Wöger, F., McBride, W., Ferayorni, A., Gregory, S., Hegwer, S., Tritschler, A., Uitenbroek, H.: 2012, The visible broadband imager: The Sun at high spatial and temporal resolution. In: Rimmele, T.R., Tritschler, A., Wöger, F., Collados Vera, M., Socas-Navarro, H., Schlichenmaier, R., Carlsson, M., Berger, T., Cadavid, A., Gilbert, P.R., Goode, P.R., Knölker, M. (eds.) Second ATST-EAST Meeting: Magnetic Fields from the Photosphere to the Corona CS-463, Astron. Soc. Pacific, San Francisco, 431. ADS.

Wöger, F., et al.: 2021, The DKIST visible broadband imager. Solar Phys. 296, in preparation. 